H. Umemura

Nagoya Math. J.

Vol. 110 (1988), 15-80

\title{
MINIMAL RATIONAL THREEFOLDS II
}

\author{
HIROSHI UMEMURA
}

The Enriques-Fano classification ([E.F], $[\mathrm{F}]$ ) of the maximal connected algebraic subgroups of the three variable Cremona group, despite of its group theoretic feature, seems to be the most significant result on the rational threefolds so far known. In this paper as in [MU] we interpret the Enriques-Fano classification from a geometric view point, namely the geometry of minimal rational threefolds. We explained in [MU] the link between the two objects; the maximal algebraic subgroups and the minimal rational threefolds. Let $(G, X)$ be a maximal algebraic subgroup of three variable Cremona group. We denote by $\mathscr{C}(G, X)$ the set of all the algebraic operations $(G, Y)$ such that $Y$ is non-singular and projective and such that $(G, Y)$ is isomorphic to $(G, X)$ as law chunks of algebraic operation: namely $(G, Y)$ is birationally equivalent to $(G, X)$. Then we define an order in $\mathscr{C}(G, X)$ : for $(G, Z),(G, W) \in \mathscr{C}(G, X),(G, Z)>(G, W)$ if there exists an $G$-equivariant birational morphism of $Z$ onto $W$.

Using the classification of [U4], we can state our result.

If $(G, X)$ is one of the maximal algebraic subgroups except for (J9) and (J11) listed in Theorem (2.1), [U4], then there exists the unique minimal element in the ordered set $\mathscr{C}(G, X)$ and any other element of $\mathscr{C}(G, X)$ is an equivariant blow-up of the minimal element. For the operations (J9) and (J11), we can describe the relatively minimal elements in $\mathscr{C}(G, X)$; there are countable many relatively minimal elements and they are explicitly constructed and are related each other by the equivariant elementary transformation. In these cases too, any other element is an equivariant blow-up of a relatively minimal elements.

Since our result thus reveals a new fascinating corner where the simplicity dominates, we have not tried to relate our result with the recent attempts of constructing minimal models for threefolds allowing

Received December 5, 1985.

Revised February 23, 1987. 
terminai singularities. Therefore there remains a very interesting problem of studying minimal elements in wider categories allowing some reasonable singularities.

As in our preceding papers, we work over the complex number field $k=\mathbf{C}$.

I should like to express my appreciation to the referee who pointed out an error in the argument of Section 8 and revealed the Euclidean model in a particular case.

\section{§1. Preliminaries}

We start with recalling the convention and some definitions.

(1.1.1) A Borel subgroup of $\mathrm{SL}_{2}$ is denoted by $B$. We denote by $D_{\infty}$ the 1-dimensional dihedral subgroup of $\mathrm{SL}_{2} ; D_{\infty}=\left\langle\left(\begin{array}{ll}t & 0 \\ 0 & t^{-1}\end{array}\right),\left(\begin{array}{cc}-1 & 1\end{array}\right)\right\rangle$, $t \in k^{*}$.

(1.1.2) An integral divisor on a variety is an irreducible reduced closed subscheme of codimension 1 .

The rational ruled surfaces appear very often in the discussion. We recall some of their properties.

(1.2) The ruled surface $\mathrm{F}_{n}(n \geq 0)$ is by definition $\mathbf{P}\left(\mathcal{O}_{\mathbf{P}_{1}} \oplus \mathcal{O}_{\mathbf{P} 1}(-n)\right)$ which has a natural $\mathbf{P}^{1}$-bundle structure $f: \mathbf{F}_{n} \rightarrow \mathbf{P}^{1}$. We denote $f^{*} \mathscr{O}_{\mathbf{P}_{1}}(m)$ by $\mathcal{O}_{\mathrm{F}_{n}}(m)$. The projection $\mathcal{O}_{\mathbf{P} 1} \oplus \mathcal{O}_{\mathbf{P} 1}(-n) \rightarrow \mathcal{O}_{\mathbf{P} 1}(-n)$ gives a section $C_{\infty}$ of $\mathrm{F}_{n} \rightarrow \mathbf{P}^{1}$. The self-intersection number $C_{\infty}^{2}=-n$ and $C_{\infty}$ is characterized by this property if $n \geq 1$ (cf. [Mar 1]). $\mathcal{O}\left(C_{\infty}\right)$ is the tautological line bundle on $\mathbf{P}\left(\mathcal{O}_{\mathbf{P} 1} \oplus \mathcal{O}_{\mathbf{P} 1}(-n)\right)$ so that $f_{*}\left(j C_{\infty}\right) \simeq S^{j}\left(\mathcal{O}_{\mathbf{P} 1} \oplus \mathcal{O}_{\mathbf{P}}(-n)\right)$ for $j \geq 0$, where $S^{j}(E)$ denotes the $j$-th symmetric tensor of $E$. We have a spectral sequence for $f$.

$\left(^{*}\right) \quad E_{2}^{p, q}=H^{p}\left(\mathbf{P}^{1}, R^{q} f_{*}\left(\mathcal{O}_{\mathrm{F}_{n}}(m) \otimes \mathcal{O}\left(j C_{\infty}\right)\right)\right)=\Rightarrow H^{*}\left(\mathrm{~F}_{n}, \mathcal{O}_{\mathrm{F}_{n}}(m) \otimes \mathcal{O}_{\mathrm{F}_{n}}\left(j C_{\infty}\right)\right)$.

For $j \geq 0, R^{q} f_{*}\left(\mathcal{O}_{F_{n}}(m) \otimes \mathcal{O}\left(j C_{\infty}\right)\right) \simeq \mathcal{O}_{\mathbf{P}_{1}}(m) \otimes R^{q} f_{*} \mathcal{O}\left(j C_{\infty}\right)$

$$
\begin{aligned}
& \simeq \mathcal{O}_{\mathbf{P} \mathbf{1}}(m) \otimes R^{q} f_{*} \mathcal{O}_{\mathbf{P}(0 \oplus \mathcal{O}(-n))}(j) \\
& \simeq\left\{\begin{array}{cl}
\mathcal{O}_{\mathbf{P} \mathbf{1}}(m) \otimes S^{j}\left(\mathcal{O}_{\mathbf{P} \mathbf{1}} \oplus \mathcal{O}_{\mathbf{P} \mathbf{1}}(-n)\right) \simeq \bigoplus_{s=0}^{j} \mathcal{O}(m-s n) & \text { if } q=0 \\
0 & \text { if } q>0,
\end{array}\right.
\end{aligned}
$$

by the projection formula. Therefore the spectral sequence $\left(^{*}\right)$ degenerates giving

$$
\left.\bigoplus_{s=0}^{j} H^{p}\left(\mathbf{P}^{1}, \mathcal{O}_{\mathrm{F}} \mathbf{1}^{\prime} m-s n\right)\right) \simeq H^{p}\left(\mathrm{~F}_{n}, \mathcal{O}_{\mathrm{F}_{n}}(m) \otimes \mathcal{O}_{\mathrm{F}_{n}}\left(j C_{\infty}\right)\right) \quad \text { for } j \geq 0
$$


(1.3.0) $\quad \mathrm{F}_{n}^{\prime}$ is by definition $\operatorname{Spec}\left(S\left(\mathcal{O}_{\mathbf{P}}(-n)\right)\right.$, where $S(E)=\bigoplus_{j \geq 0} S^{j}(E)$ denotes the symmetric algebra on $E . \quad \mathrm{F}_{n}^{\prime}$ has a natural structure of $\mathbf{A}^{1}$ bundle $f^{\prime}: \mathbf{F}_{n}^{\prime} \rightarrow \mathbf{P}^{1}$. We denote $f^{\prime *} \mathcal{O}_{\mathbf{P}_{1}}(m)$ by $\mathcal{O}_{\mathrm{F}_{n}^{\prime}}(m)$. $\mathbf{A}^{1}$-bundle $\mathbf{F}_{n}^{\prime} \rightarrow \mathbf{P}^{1}$ is the total space of line bundle $\mathcal{O}_{\mathbf{p}_{1}}(n)$ and hence we can regard as an open subvariety of $F_{n}$ : namely $\mathrm{F}_{n}-C_{\infty} \simeq \mathrm{F}_{n}^{\prime}$.

$\mathrm{F}_{n}^{\prime}$ has the 0 -section $C_{0}$ of the line bundle $\mathcal{O}_{\mathbf{P}_{1}}(n)$ which is defined in $\mathrm{F}_{n}$ by a surjective morphism $\mathcal{O}_{\mathbf{P}_{1}} \oplus \mathcal{O}_{\mathbf{P}_{1}}(-n) \rightarrow \mathcal{O}_{\mathbf{P}_{1}}$. We have $C_{0}^{2}=n$ and $C_{0}$ is disjoint from $C_{\infty}$. And conversely if we have a section $C^{\prime}$ on $\mathrm{F}_{n}$ with $C^{\prime 2}=n, C^{\prime} \cap C_{\infty}=\varnothing$ or equivalently if we have a surjective morphism $\mathcal{O}_{\mathbf{P}_{1}} \oplus \mathcal{O}_{\mathbf{P}_{1}}(-n) \rightarrow \mathcal{O}_{\mathbf{P}_{1}}$, then there is a $\mathbf{P}^{1}$-automorphism of $\mathrm{F}_{n}^{\prime} / \mathbf{P}^{1}$ (or a $\mathbf{P}^{1}$-automorphism of $\mathrm{F}_{n} / \mathbf{P}^{1}$ fixing $C_{\infty}$ ) which transforms $C$ to $C_{0}$. Since Aut ${ }_{\mathbf{P}_{1}}^{0} \mathrm{~F}_{n}^{\prime}$ is identified by Lemma (4.4), [U4] with the semi-direct product $H^{0}\left(\mathbf{P}^{1}, \mathcal{O}(n)\right) \rtimes \mathbf{G}_{m}$, the 0 -section of $\mathrm{F}_{n}^{\prime} / \mathbf{P}^{1}$ is not characteristic to $\mathrm{F}_{n}^{\prime}$ : or

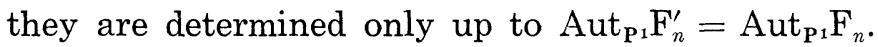

It follows from the definition $f_{*}^{\prime} \mathcal{O}_{\mathrm{F}_{n}^{\prime}} \simeq S(\mathcal{O}(-n))$. For a coherent sheaf $M$ on $\mathcal{O}_{\mathrm{F}_{n}^{\prime}}$ the spectral sequence for $f^{\prime}$ degenerates since $f$ is affine. If we write the isomorphism deduced from the degeneracy of the spectral sequence for $M=\mathcal{O}_{\mathrm{F}_{n}^{\prime}}(m)$, we get

$$
\begin{aligned}
H^{p}\left(\mathrm{~F}_{n}^{\prime}, \mathcal{O}_{\mathrm{F}_{n}^{\prime}}(m)\right) & \simeq H^{p}\left(\mathbf{P}^{1}, \mathcal{O}_{\mathbf{P}_{1}}(m) \otimes f_{*}^{\prime} \mathcal{O}_{\mathrm{F}_{n}^{\prime}}\right) \\
& \simeq H^{p}\left(\mathbf{P}^{1}, \mathcal{O}_{\mathbf{P} 1}(m) \otimes\left(\bigoplus_{j \geq 0} S^{j}\left(\mathcal{O}_{\mathbf{P}_{1}} \oplus \mathcal{O}_{\mathbf{P} 1}(-n)\right)\right)\right) \\
& \simeq \bigoplus_{j \geq 0} H^{p}\left(\mathbf{P}^{1}, \mathcal{O}_{\mathbf{P} 1}(m-j n)\right) .
\end{aligned}
$$

(1.3.2) Let $S \rightarrow \mathbf{P}^{1}$ be a $\mathbf{P}^{1}$-bundle. As the Brauer group for a curve vanishes, there exists a line bundle $E$ on $\mathbf{P}^{1}$ such that $S$ is $\mathbf{P}^{1}$-isomorphic to $\mathrm{P}(E)$. If there exists sections $D_{0}, D_{\infty}$ of $S$ such that $D_{0}$ is disjoint from $D_{\infty}$ with either $D_{0}^{2}=n$ or $D_{\infty}^{2}=-n$, then we have both $D_{0}^{2}=n$ and $D_{\infty}^{2}=-n$ and there exists a $\mathbf{P}^{1}$-isomorphism $S \simeq \mathrm{F}_{n}$ mapping $D_{0}$ (resp. $D_{\infty}$ ) to $C_{0}$ (resp. $C_{\infty}$ ) (cf. Atiyah [A]).

(1.3.3) Let $D \subset \mathrm{F}_{n}(n>0)$ be a section with $D^{2}=-n$. Hənce as we mentioned above $D=C_{\infty}$ (cf. Maruyama [Mar1] and [U3]). A non-trivial operation of $\mathrm{SL}_{2}$ on $\mathrm{F}_{n}$ gives a semi-simple part of $\mathrm{Aut}^{0} \mathrm{~F}_{n}$ and leaves invariant $C_{\infty}$ and another section $D_{0}$ disjoint from $C_{\infty}$ hence $D_{0}^{2}=n$ by (1.3.2): the $\mathrm{SL}_{2}$-orbit decomposition of $\mathrm{F}_{n}$ is (open orbit) $\cup D_{0} \cup \mathrm{D}_{\infty}$ [U3]. In particular $D_{\infty}$ (resp. $D_{0}$ ) is characterised as the $\mathrm{SL}_{2}$-invariant curve on $\mathrm{F}_{n}$ whose self-intersection number is negative (resp. positive). Conversely if we give a section $D_{0}$ with $D_{0}^{2}=n$ disjoint of $C_{\infty}$, there exist a 
unique semi-simple part of $\mathrm{Aut}^{0} \mathrm{~F}_{n}$ or equivalently non-trivial operation of $\mathrm{SL}_{2}$ on $\mathrm{F}_{n}$ leaving $D_{0}$ and $C_{\infty}$ invariant (cf. (1.3.0) and [U3]).

We consider the ordered set $\mathscr{C}(G, X)$ of equivariant completions which are birationally equivalent to a fixed operation.

Definition (1.4). For a law chunk of algebraic operation $(G, X)$, we define $\mathscr{C}(G, X)=\{(G, Y) \mid(G, Y)$ is an algebraic operation isomorphic to $(G, X)$ as law chunks of algebraic operation: in the usual language $(G, Y)$ is birationally equivalent to $(G, X) . \quad Y$ is non-singular and projective (cf. [U1], [MU]).

$\mathscr{C}(G, X)$ is non-empty by [Su] if $G$ is linear, in particular if $X$ is rational (see Theorem (3.2), [U2]) (see also [U5]). We define an order $>$ in $\mathscr{C}(G, X)$.

Definition (1.5). For $\left(G, X_{1}\right), \quad\left(G, X_{2}\right) \in \mathscr{C}(G, X), \quad\left(G, X_{1}\right)>\left(G, X_{2}\right)$ if there exists a $G$-equivariant birational morphism of $X_{1}$ onto $X_{2}$.

Our result is a fruitful application of the theory of extremal rays due to Mori, which is a generalization of the classical theory of both ruled surfaces and of exceptional divisors of the first kind over a surface. Referring the reader to [Mo] for the detail, we recall briefly the framework of his theory and indicate how we can apply it. Let $N(X)$ be the $\mathbf{R}$-vector space of all numerical equivalence classes of 1-cycles over a non-singular projective threefold $X$ with coefficients in $\mathbf{R}$ and $N E(X)$ be the smallest convex cone in $N(X)$ containing all effective 1-cycles closed under the multiplication by $\mathbf{R}_{+}$. We denote by $\overline{N E}(X)$ the closure of $N E(X)$ in the $\mathbf{R}$-vector space $N(X)$. A half line $R=\mathbf{R}_{+}[Z]$ in $\overline{N E}(X)$ is called an extremal ray if (i) $\left(Z, c_{1}(X)\right)>0$ and if (ii) for $Z_{1}, Z_{2} \in \overline{N E}(X)$, $Z_{1}+Z_{2} \in R$ implies $Z_{1}, Z_{2} \in R$.

THEOREM (Mori [Mo]). Let $X$ be a non-singular projective threefold and $R \subset \overline{N E}(X)$ be an extremal ray. Then there exists a morphism $\phi: X \rightarrow Y$ to a projective variety $Y$ such that (i) $\phi_{*} \mathcal{O}_{X}=\mathcal{O}_{Y}$ and (ii) for any irreducible curve $C$ in $X,[C] \in R$ if and only if $\operatorname{dim} \phi(C)=0$.

The structure of the morphism $\phi$ is analysed in Theorem (3.3), Corollary (3.4) and Theorem (3.5), [Mo]. Roughly speaking we have one of the following: (i) $\phi$ is a blow-down, (ii) $\phi$ makes $X$ into a fibration over a surface or a curve, (iii) $X$ is a Fano threefold with $\rho(X)=1$.

Since the morphism $\phi$ is functorial, if an algebraic group $G$ operates 
on $X$, then $G$ operates also on $Y$ such that $\phi$ is $G$-equivariant. We apply this for an operation $(G, X)$ of a linear algebraic group on a non-singular projective threefold $X$ such that $(G, X)$ gives a maximal connected algebraic subgroup of the three variable Cremona group. In most of our applications, $Y$ is automatically smooth and we get one of the following:

(1) $\phi$ is an equivariant blow-up of non-singular $Y$,

(2) $\phi$ makes $X$ into an equivariant $\mathbf{P}^{1}$-bundle over a rational ruled surface,

(3) $\phi$ makes $X$ into a del Pezzo bundle over $\mathbf{P}^{1}$,

(4) $X$ is a Fano threefold with $\rho(X)=1$.

In the case (1), we apply the Mori theory again to $Y$ and continue to look for a minimal model. In the cases (2) and (3), we can determine the structure of $X$ completely. The case (4) rarely happens and in fact never when we limit ourselves to the de Jonquières type operations $(G, X)$ : recall that $(G, X)$ is of de Jonquières type if there exist an operation $(G, Y), \operatorname{dim} Y=1$ or 2 and a dominant $G$-equivariant rational $\operatorname{map} X \cdots Y$.

(1.6) We mean by a blow-up $X \rightarrow Y$ a blow-up of a non-singular variety $Y$ at a non-singular irreducible center. But by abuses of language, we often call a sequence of blow-ups $f_{i}$ also a blow-up: $f=f_{1} \circ f_{2} \circ \cdots \circ f_{n}$ : $X_{n} \rightarrow X_{0}$, where $f_{i+1}: X_{i+1} \rightarrow X_{i}$ is a blow-up of $X_{i}$ at a non-singular irreducible center $(0 \leq i \leq n-1)$. We shall distinguish them clearly to avoid the confusion.

The following theorem is useful when we analyse the elements of $\mathscr{C}(G, X)$.

Theorem (Danilov [Da]) (1.7). Let $f: X \rightarrow Y$ be a birational morphism of non-singular projective varieties. If $\operatorname{dim} f^{-1}(y) \leq 1$ for any point $y \in Y$, then up to an automorphism of $X, f$ can be decomposed into a sequence of blow-ups with smooth centers of codimension 2.

When $\operatorname{dim} X=\operatorname{dim} Y=2$, the Theorem is classical and well-known. We use the Theorem for threefolds.

We need the following Lemma which is finer than Lemma (1.21), [U3].

Lemma (1.8). Let $f: X \rightarrow Y$ be a projective, flat morphism of algebraic varieties. Let $\psi: G \times X \rightarrow X$ be an operation of a reductive algebraic group $G$ on $X$ such that $f \circ \psi=f \circ p_{2}$, where $p_{2}: G \times X \rightarrow X$ is the projec- 
tion. Then the following conditions are equivalent.

(1) $(G, X)$ is effective (resp. almost effective).

(2) There exists a point $y_{0} \in Y$ such that the induced operation $\left(G, f^{-1}\left(y_{0}\right)\right)$ is effective (resp. almost effective).

(3) For any point $y \in Y$, the induced operation $\left(G, f^{-1}(y)\right)$ is effective (resp. almost effective).

Proof. The implications $(3) \Rightarrow(2) \Rightarrow(1)$ are evident. Thus we have to prove $(1) \Rightarrow(3)$. This follows from Exposé IX, S.G.A.D. but we prove this directly. First we prove $(1) \Rightarrow(3)$ for the almost effective case. We show that if (3) does not hold for $(G, X)$, then (1) does not hold. If there exists a point $y_{0} \in Y$ such that $\left(G, f^{-1}\left(y_{0}\right)\right)$ is not almost effective, then there exists a positive dimensional normal subgroup of $G$ which operates trivially on $f^{-1}(y)$. Since any positive dimensional normal subgroup of the reductive group $G$ contains a non-trivial torus, we can thus find a torus $T \neq 1 \subset G$ which operates trivially on $f^{-1}\left(y_{0}\right)$. It is sufficient to show that $(T, X)$ is trivial. Namely to prove $(1) \Rightarrow(3)$, it is sufficient to prove it for the following special case: if $G$ is a torus $T$ and if there exists a point $y_{0} \in Y$ such that $T$ operates on $f^{-1}(y)$ trivially, then $(T, X)$ is trivial. Let $L$ be a relatively ample line bundle for $f: X \rightarrow Y$ such that $f_{*} L$ is locally free of finite rank and $R^{i} f_{*} L=0$ for all $i \geq 1$ : such a bundle $L$ exists replacing $L$ by $L^{\otimes n}, n \gg 0$ if necessary. $\psi$ defines a morphism $\tilde{\psi}: G \times Y=T \times Y \rightarrow \operatorname{Aut}_{Y} \mathbf{P}\left(f_{*} L\right)$ of group schemes over $Y$. For a point $y_{0} \in Y$, we can find an affine neighbourhood $Y^{\prime}$ of $y_{0}$ such that $f_{*} L$ is a free $\mathcal{O}_{Y^{\prime}}$-Module. Therefore we may assume that $Y$ is affine. Then $\tilde{\psi}$ gives over $Y=\operatorname{Spec} A$ a morphism $\tilde{\psi}^{\prime}: T \times Y \rightarrow \mathrm{PGL}_{n} \times Y$. Using embedding $\mathrm{PGL}_{n} \longrightarrow \mathrm{GL}_{N}$, we finally get $\phi: T \times Y \rightarrow \mathrm{GL}_{N} \times Y$ and have to prove that $\phi$ is trivial. Putting by $T_{\ell^{n}}=\left\{t \in T \mid t^{\ell^{n}}=1\right\}$ the finite subgroup of elements of order $\ell^{n}, \phi$ defines a $T_{\ell^{n}}$-module $M=A^{\oplus N}$, where $\ell$ is a prime number (different from $p$ if $\operatorname{ch} k=p>0$, which is not the case). The character $\chi: T_{\ell^{n}} \rightarrow A$ is a function of an element $t$ of $T_{\ell^{n}}$ and of a point $y$ of $Y: \chi(t ; y)$. If we fix $t \in T_{\ell^{n}}, y \mapsto \chi(t ; y)$ is regular function on $Y$. Since $T_{\ell^{n}}$ is finite, there are only finitely many representations of $T_{\ell^{n}}$ of rank $N$ over $k$ and hence the function $y \mapsto \chi(t ; y)$ is constant. Namely for all $y \in Y$, the reduction of $T_{\ell^{n}}$-module $M$ at $y$ is isomorphic each other. Since the reduction at $y_{0}$ is the trivial $T_{\ell^{n}}$-module, $T_{\ell^{n}}$ operates trivial on each fibre. Taking all $n>0, \cup T_{\ell^{n}}$ is dense in $T_{\ell n}$, we conclude $T$ operates 
trivial on $M$. Hence $\phi, \tilde{\psi}^{\prime}$ are trivial. The proof $(1) \Rightarrow(3)$ for effectiveness follows from the last argument for finite groups.

Lemma (1.8) is the rigidity of operation of a reductive group.

We prove 2 lemmas for the automorphism group of $\mathbf{A}^{1}$-bundle. As we explained above $\mathbf{A}^{1}$-bundles appear very often in the sequel.

LEMMA (1.9). Let $L$ be a line bundle over a projective algebraic variety $X$ and $D$ an effective Cartier divisor on $X$. (a) Then we denoting by $L$ the total space $\operatorname{Spec}\left(\oplus_{i \geq 0} L^{\otimes-i}\right)$ of the line bundle $L, \operatorname{Aut}_{X} L$ is representable in the category of reduced schemes over $k$ and $\operatorname{Aut}_{X}^{0} L$ is isomorphic to the semi-direct product $H^{0}(X, L) \rtimes \mathbf{G}_{m}$. (b) If the inclusion $H^{0}(X, L(-D)) \rightarrow H^{0}(X, L)$ is bijective, then $H^{0}(X, L)$ fixes all the points of $L$ lying over $D$.

Proof. For any reduced scheme $T \rightarrow X, \alpha \in \mathrm{Aut}_{T}\left(T \times_{X} L\right)$ is locally an affine transformation and hence can be extended to an $T$-automorphism of the $\mathbf{P}^{1}$-bundle $T \times_{X} \mathbf{P}\left(\mathcal{O} \oplus L^{-1}\right)$. Therefore Aut $_{T}\left(T \times_{X} L\right)=$ $\left\{\sigma \in \operatorname{Aut}_{T}\left(T \times{ }_{X} \mathbf{P}\left(\mathcal{O} \oplus L^{-1}\right)\right)\right.$ leaving the $\infty$-section invariant $\}$, which is a closed subgroup of the automorphism group Aut $\mathbf{P}\left(\mathcal{O} \oplus L^{-1}\right)$ of the projective variety $\mathbf{P}\left(\mathcal{O} \oplus L^{-1}\right)$. Let $\bigcup_{\alpha \in I} U_{\alpha}$ be a covering of $X$ such that $L$ is defined by a 1-cocycle $\left\{g_{\alpha \beta}\right\}_{\alpha, \beta \in I}, g_{\alpha, \beta} \in H^{0}\left(U_{\alpha} \cap U_{\beta}, \mathcal{O}^{*}\right)$. Let $s \in H^{0}(X, L)$ which is locally given by $s_{\alpha} \in H^{0}\left(U_{\alpha}, 0\right)$ such that $g_{\alpha \beta} s_{\alpha}=s_{\beta}$. $L$ is an $\mathbf{A}^{1}$-bundle gluing $U_{\alpha} \times \mathbf{A}^{1}(\alpha \in I)$ by $g_{\alpha \beta}:\left(x, u_{\alpha}\right) \in U_{\alpha} \times \mathbf{A}^{1}$ and $\left(y, u_{\beta}\right) \in U_{\beta} \times \mathbf{A}^{1}$ are identified if $x=y$ and $g_{\alpha \beta} u_{\alpha}=u_{\beta}$. Thus the automorphism $\left(x, u_{\alpha}\right) \mapsto$ $\left(x_{1} u_{\alpha}+s_{\alpha}\right)$ of $U_{\alpha} \times \mathbf{A}^{1}$ defines an action of the vector group $H^{0}(X, L)$ and $\mathbf{G}_{m}$ operates on each fibre by the scalor multiplication. If we notice that the $\operatorname{Lie}\left(\operatorname{Aut}_{X} L\right) \cong\{f \in \operatorname{End}(\mathcal{O} \oplus L) \mid f(\mathcal{O} \oplus 0) \subset \mathcal{O} \otimes 0\} /$ scalor multiplications, (a) follows from Lemma (1.8). If $H^{0}(X, L(-D)) \cong H^{0}(X, L)$, all the section of $H^{\circ}(X, L)$ vanish on $D$ and (b) is proved.

A similar argument gives

LEMma (1.10). Let $L$ be a line bundle over a projective variety $X$ and $D$ an effective Cartier divisor on $X$. (a) We denoting by $Z$ an $\mathbf{A}^{1}$-bundle over $X$ defined by a non-trivial extension $0 \rightarrow \mathcal{O} \rightarrow \widetilde{\mathscr{E}} \rightarrow L \rightarrow 0, \operatorname{Aut}_{X} Y$ is representable in the category of reduced schemes and Aut $_{X}^{0} Y$ is isomorphic to the vector group $H^{0}(X, L)$. (b) If the inclusion $H^{0}(X, L(-D)) \rightarrow H^{0}(X, L)$ is bijective, then Aut ${ }_{X}^{0} Z$ fixes all the points of $Z$ lying over $D$.

Proof. This is proved by the same method as Lemma (1.9). See also 
Lemma (4.22) [U4].

Certain $\mathrm{SL}_{2}$-actions on a rational threefold do not have a fixed point as we see in [MU] and shall see later. In general, an $\mathrm{SL}_{2}$-action on a threefold does not have too many fixed points.

LEMMA (1.11). Let $\left(\mathrm{SL}_{2}, X\right)$ be an operation of $\mathrm{SL}_{2}$ on a non-singular threefold $X$. Then the dimension of the subvariety $S$ of $\mathrm{SL}_{2}$-fixed points is at most 1 unless $X=S$.

Proof. Assume that $\mathrm{SL}_{2}$-fixes all the point of a surface $S$ on $X$ and that $\left(\mathrm{SL}_{2}, X\right)$ is non-trivial i.e. $X \neq S$. Let $x \in S$ be a smooth point of $S$. $\mathrm{SL}_{2}$ operates on the Zariski tangent space $m_{x} / m_{x}^{2}$ but this operation fixes the $S$-direction so that $m_{x} / m_{x}^{2}$ contains a trivial representation $k \oplus k$ of degree 2. Hence by the complete reducibility of $\mathrm{SL}_{2}, m_{x} / m_{x}^{2}$ is a trivial $\mathrm{SL}_{2}$-module. As we have $S^{j}\left(m_{x} / m_{x}^{2}\right) \simeq m_{x}^{j} / m_{x}^{j+1}, m_{x}^{j} / m_{x}^{j+1}$ is a trivial $\mathrm{SL}_{2}$ module. Therefore by the complete irreducibility. $\mathcal{O}_{x} / m_{x}^{j}$ is a trivial $\mathrm{SL}_{2}$ module for $j \geq 1$. Consequently $\hat{\mathcal{O}}_{x}=\lim _{j} \mathcal{O}_{x} / m_{x}^{j}$ is a trivial $\mathrm{SL}_{2}$-module and $\mathrm{SL}_{2}$ operates on $\mathcal{O}_{x}$ trivially and hence on its quotient field, which is the function field of $X$. This is absurd as we assumed that $\left(\mathrm{SL}_{2}, X\right)$ is non-trivial.

The following Lemma gives an obstruction for blowing-down a divisor to a smooth point.

Lemma (1.12). Let $\varphi: X \rightarrow Y$ be a birational morphism of smooth projective threefolds and $D \subset X$ be an irreducible subvariety of $X$. If the image $\varphi(D)$ is a point, and if $\varphi$ induces an isomorphism $X-D \simeq Y-\varphi(D)$, then $\mathcal{O}(D) \otimes \mathcal{O}_{D}$ is an ample line bundle on $D$.

Proof. Let $A$ be an ample divisor on $X . \varphi^{*} \varphi_{*} A$ is linearly equivalent to $A+n D$ with $n>0$. Since for any divisor $B$ on $Y, \mathcal{O}\left(\varphi^{*} B\right) \otimes \mathcal{O}_{D}$ is trivial on $D, \varphi^{*} \varphi_{*} A=A+n D$ is trivial when restricted on $D$. Thus $\mathcal{O}(A) \otimes \mathcal{O}(n D) \otimes \mathcal{O}_{D} \simeq \mathcal{O}_{D}$ hence $\mathcal{O}(A) \otimes \mathcal{O}_{D} \simeq \mathcal{O}(-n D) \otimes \mathcal{O}_{D}$ and $\mathcal{O}(-D) \otimes \mathcal{O}_{D}$ is ample.

The following Lemma would be well-known among the specialists. For the definition of a conic boudle, see [Be].

Lemma (1.13). Let $(\varphi, f):(G, X) \rightarrow(G, Y)$ be a morphism of algebraic operations such that $f: X \rightarrow Y$ is a conic bundle over a non-singular surface $Y$ and $\rho(X)=\rho(Y)+1$. If any reduced G-invariant curve on $Y$ is isomorphic to the disjoint union of some $\mathbf{P}^{1}$ 's, then $f: X \rightarrow Y$ is a $\mathbf{P}^{1}$-bundle. 
Proof. Let $C \subset Y$ be an irreducible component of discriminant locus of the conic curve. We prove $C=\varnothing$. For otherwise it follows from the assumption that $\rho(X)=\rho(Y)+1$ and from p. 87, Lemma, [Mi] that $\varphi^{-1}(C)$ is irreducible. Therefore by Proposition 1.5, [Be], there exists an étale 2-covering $C^{\prime} \rightarrow C$ with an irreducible $C^{\prime}$. This impossible since by hypotheses $C \simeq \mathbf{P}^{1}$.

\section{§. Compact case}

Let $(G, X)$ denote one of the following operations of Theorem (2.1), [U4]: (P1) $\left(\mathrm{PGL}_{4}, \mathbf{P}^{3}\right),(\mathrm{P} 2)\left(\mathrm{PSO}_{5}\right.$, quadric $\left.\subset \mathbf{P}^{4}\right),(\mathrm{J} 1)\left(\mathrm{PGL}_{2} \times \mathrm{PGL}_{3}, \mathbf{P}^{1} \times \mathrm{P}^{2}\right)$, (J2) $\left(\mathrm{PGL}_{2} \times \mathrm{PGL}_{2} \times \mathrm{PGL}_{2}, \mathbf{P}^{1} \times \mathbf{P}^{1} \times \mathrm{P}^{1}\right)$ and $(\mathrm{J} 4)\left(\mathrm{PGL}_{3}, \mathrm{PGL}_{3} / B\right)$. These are the maximal subgroups where $X$ is a projective homogeneous space. Let $(G, Y) \in \mathscr{C}(G, X)$. Then by definition, there exists a $G$-equivariant birational map $f: X \cdots Y$ which should be biregular as $X$ is a complete homogeneous space. Therefore we have proved.

TheOREm (2.1). Let $(G, X)$ be one of the operations (P1), (P2), (J1), (J2) and (J4) of Theorem (2.1), [U4]. Then the set $\mathscr{C}(G, X)$ consists of a single element.

\section{§3. Equivariant completions of J3}

$(\mathrm{J} 3)$ is the operation $\left(\mathrm{PGL}_{2} \times\right.$ Aut $\left.^{0} \mathrm{~F}_{m}^{\prime}, \mathrm{P}^{1} \times \mathrm{F}_{m}^{\prime}\right)=(G, X)(m \geq 2)$ which has a projective non-singular compactification $(G, Y)=\left(\mathrm{PGL}_{2} \times \mathrm{Aut}^{0} \mathrm{~F}_{m}\right.$, $\left.\mathbf{P}^{1} \times \mathrm{F}_{m}\right)$. The orbit decomposition is $\mathbf{P}^{1} \times \mathrm{F}_{m}^{\prime} \cup\left(\mathbf{P}^{1} \times \mathbf{F}_{m}-\mathbf{P}^{1} \times \mathrm{F}_{m}^{\prime}\right)$. The latter is a divisor on $\mathbf{P}^{1} \times \mathrm{F}_{m}$, which we denote by $D$. Let $(G, Z) \in \mathscr{C}(G, X)$. Then we have a $G$-equivariant birational map $f: Y \cdots Z$. By Hironaka's theorem of equivariant resolution, we can blow-up $Y$ equivariantly to eliminate the indeterminacy of $f$. But as there is no orbit of codimension $\geq 2, f$ should be a birational morphism inducing an isomorphism between the open orbits. We show that $f$ is biregular. Assume that $f(D)$ is a curve. Then it follows from Theorem (1.7), $f$ is a blow-up but this is impossible since $D \simeq \mathbf{P}^{1} \times \mathbf{P}^{1}$ and $\mathcal{O}(D) \otimes \mathcal{O}_{D}$ is $\mathcal{O}_{\mathbf{P}^{1} \times \mathbf{P}^{1}}(0,-m), m \geq 2$. It follows from Lemma (1.12) that $f(D)$ is not a point since $\mathcal{O}(D) \otimes \mathcal{O}_{D} \simeq$ $\mathcal{O}_{\mathbf{P} 1 \times \mathbf{p}_{1} 1}(0,-m), m \geq 2$. Therefore it follows from Zariski's main theorem that $f$ is biregular. Hence we have proved.

Theorem (3.2). The set $\mathscr{C}\left(\mathrm{PGL}_{2} \times\right.$ Aut $\left.^{0} \mathrm{~F}_{m}^{\prime}, \mathrm{P}^{1} \times \mathrm{F}_{m}^{\prime}\right)(m \geq 2)$ consists of a single element. 


\section{§4. Equivariant completions of J5}

(J5) is the operation $\left(\mathrm{PGL}_{2}, \mathrm{PGL}_{2} / D_{2 n}\right)$, where $D_{2 n}$ is the dihedral subgroup of order $2 n$ with $n \geqq 4$. We denote by $\tilde{D}_{2 n}$ the binary dihedral subgroup of order $4 n$ of $\mathrm{SL}_{2}: \tilde{D}_{2 n}$ is the subgroup of $\mathrm{SL}_{2}$ generated by $\left(\begin{array}{ll}-1 & 1\end{array}\right)$ and $\left(\begin{array}{ll}\zeta & 0 \\ 0 & \zeta^{-1}\end{array}\right), \zeta^{2 n}=1$. Since $(G, W)=\left(\mathrm{SL}_{2}, \mathrm{SL}_{2} / \tilde{D}_{2 n}\right)$ almost effectively realizes $\left(\mathrm{PSL}_{2}, \mathrm{SL}_{2} / \tilde{D}_{2 n}\right)=\left(\mathrm{PGL}_{2}, \mathrm{PGL}_{2} / D_{2 n}\right)$, we study $\mathscr{C}(G, W)$. Sometimes $\mathscr{C}(G, W)$ is denoted by $\mathscr{C}(\mathrm{J} 5: n)$.

The following Lemma was proved in [MU] but we give a proof because later we need a more general assertion which one can prove easily once one review the following

Lemma (4.1). Let $\left(\mathrm{SL}_{2}, X\right)$ be an operation of $\mathrm{SL}_{2}$ on a projective nonsingular threefold $X$. If $\mathrm{SL}_{2}$ has an open orbit on $X$, then there is no $\mathrm{SL}_{2}$-fixed point on $X$. In particular if $(G, Y) \in \mathscr{C}(\mathrm{J} 5 ; n)$, then there is no G-fixed point on $Y$.

Proof. Let $P \in Y$ and assume that $P$ is a fixed point of $G$. Since $Y$ is projective and $G=\mathrm{SL}_{2}$ is simple, there exists an $\mathrm{SL}_{2}$-invariant affine neighbourhood Spec $A$ of $P$. We denote by $I$ the ideal of $A$ consisting of the elements of $A$ vanishing at $P$. Putting $m=I A_{I}$, we get a surjective $\mathrm{SL}_{2}$-linear map $\varphi: I^{2} \rightarrow m^{2} / m^{3}$. We can choose an $\mathrm{SL}_{2}$-invariant finite dimensional subspace $V$ of $I^{2}$ such that $\varphi(V)=m^{2} / m^{3}$ by (1.9) Proposition, [Bo]. The Zariski tangent space $m / m^{2}$ is an $\mathrm{SL}_{2}$-module and $S^{2}\left(m / m^{2}\right) \simeq$ $m^{2} / m^{3}$. Thus $m^{2} / m^{3}$ contains a non-zero $\mathrm{SL}_{2}$-invariant $\bar{f}$. For, there certainly exists $\bar{f}$ if $m / m^{2}$ is reducible (which implies that $m / m^{2}$ contains a trivial representation since $m / m^{2}$ is 3-dimensional) and if $m / m^{2}$ is irreducible, $\mathrm{m} / \mathrm{m}^{2}$ is isomorphic to the vector space of homogeneous polynomials of degree 2 in $x, y$ where $\mathrm{SL}_{2}$ operates on $x, y$ in usual way and the discriminant which is a polynomial of degree 2 in the coefficients of a given homogeneous polynomial, is the $\mathrm{SL}_{2}$-invariant. Since the image $\varphi(V)=m^{2} / m^{3}$ contains a trivial representation $k \bar{f}$, by the complete reducibility of $\mathrm{SL}_{2}$, we can find a non-zero lifting $f \in V$ of $\bar{f}$ which is $\mathrm{SL}_{2}$ invariant hence constant as $\mathrm{SL}_{2}$ has an open orbit. This is absurd since $f$ vanishes at $P$.

To apply the main Theorem of [Mo], we need a more general assertion than Lemma (4.1). 
Lemma (4.2). Let $(G, X)$ be an (algebraic) operation of a reductive algebraic group $G$ on an affine variety $X=\operatorname{Spec} A$. Let $P \in X$ be a fixed point, $n$ a non-negative integer and let $W$ be a G-invariant subspace of $I^{n} / I^{n+1}$, where $I$ is the ideal of $A$ consisting of regular functions on $X$ vanishing at $P$. Then there exists a G-invariant subspace $W^{\prime}$ of $I^{n}$ such that the canonical map $W^{\prime} \rightarrow W^{\prime}+I^{n+1} / I^{n+1} \rightarrow I^{n} / I^{n+1}$ induces a G-isomorphism of $W^{\prime}$ and $W$.

Proof. The lemma is proved by the same method as in the proof of Lemma (4.1).

Corollary (4.3). Let $(G, X), P$ and $n$ be as in Lemma (4.2). If there exists a G-invariant element $\bar{f}$ in $I^{n} / I^{n+1}$, then there is a G-invariant lifting $f$ of $\bar{f}$ to $I^{n}$.

Proof. This is a particular case of Lemma (4.2) since $G$ is completely reducible.

Lemma (4.4). Let $(G, X) \in \mathscr{C}(\mathrm{J} 5 ; n) . \quad$ Then the 4 cases $(3.3 .2) \cdots(3.3 .5)$ for $X$ in Theorem (3.2), [Mo] never occur.

Proof. We excluded the case (3.3.2) by Lemma (4.1) since $\mathrm{SL}_{2}$-operates on $Y$. The case (3.3.4) does not occur neither. For otherwise, the divisor $D$ to be mapped to a singular point is $\mathrm{SL}_{2}$-invariant and the single singular point of the cone $D$ is left fixed by $\mathrm{SL}_{2}$, which contradicts Lemma (4.1). Assume that the case (3.3.3) happens. Then $D \simeq \mathbf{P}^{1} \times \mathbf{P}^{1}$ and $\mathcal{O}_{D}(D)$ is of bidegree $(-1,-1)$. Let $\phi: X \rightarrow Y$ be the morphism arising from the Mori theory and $\phi(D)=Q$. $\mathrm{SL}_{2}$-operates on $Y$ and $\phi$ is equivariant. It follows from Lemma (3.3.2), [Mo] that $I_{Q} / I_{Q}^{2} \simeq H^{0}\left(\mathcal{O}_{D}(-D)\right) \simeq H^{0}\left(\mathbf{P}^{1} \times \mathbf{P}^{1}, \mathcal{O}(1,1)\right)$. Thus the Zariski tangent space $I_{Q} / I_{Q}^{2}$ at $Q$, as an $\mathrm{SL}_{2}$-module, is isomorphic to the tensor product $M_{1} \otimes M_{2}$. Thus we have either (1) $M_{1}$ and $M_{2}$ are irreducible or (2) $M_{1}$ or $M_{2}$ is trivial. The first case never occurs. For otherwise since $Y$ is projective and $\mathrm{SL}_{2}$ is simple, we can find an $\mathrm{SL}_{2}$ invariant affine neighbourhood $\operatorname{Spec} A$ of $Q$. Since there is a non-zero $\mathrm{SL}_{2}$-invariant in $I_{Q} / I_{Q}^{2}$ in case (1), it follows from Corollary (4.3) that we can find a non-zero $\mathrm{SL}_{2}$-invariant meromorphic function regular on Spec $A$ vanishing at $Q$. This is absurd since $\mathrm{SL}_{2}$ has an open orbit on $Y$. In case (2) we can find as before an $\mathrm{SL}_{2}$-invariant affine neighbourhood Spec $A$ of $Q$. Since there is a 2-dimensional $\mathrm{SL}_{2}$-invariant subspace in $I_{Q} / I_{Q}^{2}$ in case (2), it follows that we can find a 2-dimensional $\mathrm{SL}_{2}$-invariant 
subspace of $A$ by Lemma (4.2) hence an $\mathrm{SL}_{2}$-invariant linear system of dimension 1. Namely we get an $\mathrm{SL}_{2}$ equivariant dominant rational map $X \cdots \mathbf{P}^{1}$, hence in particular a morphism $\left(\mathrm{SL}_{2}, \mathrm{SL}_{2} / \tilde{D}_{2 n}\right) \rightarrow\left(\mathrm{SL}_{2}, \mathbf{P}^{1}\right)$ of algebraic operations from the open orbit. This is impossible as $\tilde{D}_{2 n}$ can not be contained in a Borel subgroup. Now assume that the case (3.3.5) occurs. We argue similarly, using the notation in Mori [Mo], p. 146-147 as above. It follows from Lemma (3.32) that $I_{Q} / I_{Q}^{2} \simeq H^{0}\left(D, \mathcal{O}_{D}(-D)\right) \simeq$ $H^{0}\left(\mathbf{P}^{2}, \mathcal{O}(2)\right)$. The latter is isomorphic to $S^{2}\left(H^{0}\left(\mathbf{P}^{2}, \mathcal{O}(1)\right)\right.$ hence contains a non-zero $\mathrm{SL}_{2}$-invariant element $\bar{f}$ as we see in the Proof of Lemma (4.1). Arguing as in the preceding cases, $\bar{f}$ yields a non-zero $\mathrm{SL}_{2}$-invariant (hence constant) rational function $h$ vanishing at $Q$.

Let $\left(\mathrm{SL}_{2}, \mathbf{P}^{1} \times \mathbf{P}^{1}\right)$ be the diagonal operation and $E$ denote the irreducible SL-module of dimension 3. Then $\mathrm{SL}_{2}$ operates on $\mathbf{P}(E)$ giving an operation $\left(\mathrm{SL}_{2}, \mathbf{P}^{2}\right)$. $\mathrm{SL}_{2}$ has an open orbit on $\mathbf{P}^{2}$ and the orbit decomposition of $\mathbf{P}^{2}$ is $\mathrm{SL}_{2} / D_{\infty} \cup \mathrm{SL}_{2} / B$, where $B$ is a Borel subgroup and $D_{\infty}$ is a subgroup generated by $\left(\begin{array}{ll}a & \\ & a^{-1}\end{array}\right), a \in k^{*}$ and $\left(\begin{array}{cc}-1 & 1\end{array}\right)$. The latter orbit is a quadric in $\mathbf{P}^{2}$. Let us define $\varphi: \mathbf{P}^{1} \times \mathbf{P}^{1} \rightarrow \mathbf{P}^{2}$ by $\varphi((x, y),(u, v))=$ $(x u, y u+x v, y v) \in \mathbf{P}^{2}$ for $((x, y),(u, v)) \in \mathbf{P}^{1} \times \mathbf{P}^{1}$. Then $\varphi: \mathbf{P}^{1} \times \mathbf{P}^{1} \rightarrow \mathbf{P}^{2}$ is an $\mathrm{SL}_{2}$-equivariant 2-covering whose branch locus is the diagonal of $\mathbf{P}^{1} \times \mathbf{P}^{1}$ and whose ramification locus is the quadric $X_{1}^{2}-4 X_{0} X_{2}=0$ in $\mathbf{P}^{2}$. Let $\mathcal{O}(i, j)$ denote the line bundle over $\mathbf{P}^{1} \times \mathbf{P}^{1}$ of bidegree $i, j$. The direct image sheaf $\varphi_{*} \mathcal{O}(i, j)=E(i, j)$ is the rank 2 vector bundle studied by Schwartzenberger [Sc]. $\mathrm{SL}_{2}$ operates on $E(i, j)$ hence on $\mathbf{P}(E(i, j))$. Since $\varphi^{*} \mathcal{O}_{\mathbf{P}_{2}}(1)$ is isomorphic to $\mathcal{O}(1,1), E(i, j) \simeq E(i-j, 0) \otimes \varphi^{*} \mathcal{O}_{\mathbf{P}_{2}}(j)$ hence $\mathbf{P}(E(i, j)) \simeq \mathbf{P}(E(i-j, 0))$. Thus we denote by $E_{i}$ the vector bundle $E(i, 0)$.

Lemma (4.5). $\mathrm{SL}_{2}$ operates on $\mathbf{P}\left(E_{n}\right)$ and has an open orbit isomorphic to $\mathrm{SL}_{2} / \tilde{D}_{2 n}$ for $n \geqq 1$.

Proof. The stabilizer $H$ at $(0,1,0) \in \mathbf{P}^{2}$ is the one dimensional subgroup $D_{\infty}$ of $\mathrm{SL}_{2}$ generated by $\left(\begin{array}{ll}t & 0 \\ 0 & t^{-1}\end{array}\right) \in \mathrm{SL}_{2}, t \in k^{*}$ and $\left(\begin{array}{cc}1 & 1 \\ -1 & \end{array}\right) . \varphi^{-1}(0,1,0)=$ $((0,1),(1,0)) \cup((1,0),(0,1))$ and the fibre of $E_{n}$ at $(0,1,0)$ is identified with the direct sum of the fibre of $\mathcal{O}(n, 0)$ at $((0,1),(1,0))$ and $((1,0),(0,1))$. The operations of $T=\left\{\left(\begin{array}{ll}t & 0 \\ 0 & t^{-1}\end{array}\right) \mid t \in k^{*}\right\}$ on the fibres of $\mathcal{O}(n, 0)$ at $((0,1)$, $(1,0))$ and $((1,0),(0,1))$ are respectively by $t^{n}$ and $t^{-n}$. The operation of $\left(\begin{array}{ll}-1 & 1\end{array}\right)$ on $\mathbf{P}^{1} \times \mathbf{P}^{1}$ interchanges the points $((0,1),(1,0))$ and $((1,0),(0,1))$ 
hence the fibres of $\mathcal{O}(n, 0)$. Thus the stabilizer at a suitable point of $\mathbf{P}$ (the fibre of $E_{n}$ at $\left.(0,1,0)\right)$ is $\tilde{D}_{2 n}$.

Lemma (4.6). Let $(G, X) \in \mathscr{C}(\mathrm{J} 5 ; n),(G, Y)$ be an algebraic operation and $f: X \rightarrow Y$ be a G-equivariant dominant rational map. If $Y$ is nonsingular and complete and if $\operatorname{dim} Y \leqq 2$, then $(G, Y)$ is isomorphic to $\left(\mathrm{SL}_{2}, \mathbf{P}^{2}\right)$ where $\mathrm{SL}_{2}$ operates on $\mathbf{P}^{2}$ through the irreducible representation of degree 3 .

Proof. Assume that $Y$ is a curve. As $X$ is rational, $Y$ is isomorphic to $\mathbf{P}^{1}$ by the Lüroth theorem and $\left(\mathrm{SL}_{2}, Y\right)$ is the usual operation of $\mathrm{SL}_{2}$ on $\mathbf{P}^{1}$ since $\mathrm{SL}_{2}$ must have an open orbit on $Y$. Thus $f$ defines a surjective morphism $\mathrm{SL}_{2} / \tilde{D}_{2 n} \rightarrow \mathrm{SL}_{2} / B$. Namely up to conjugacy $\tilde{D}_{2 n}$ is contained in the Borel subgroup $B$ of $\mathrm{SL}_{2}$. This is absurd because $\tilde{D}_{2 n} \subset \mathrm{SL}_{2}$ is anirreducible representation of degree 2 of $\tilde{D}_{2 n}$. Hence $Y$ is a surface. $G$ has an open orbit also on $Y$ since $(G, X)$ has the open orbit and $f$ is dominant. Let the open orbit on $Y$ be isomorphic to $\mathrm{SL}_{2} / H$. Then there is a surjective morphism $\mathrm{SL}_{2} / \tilde{D}_{2 n} \rightarrow \mathrm{SL}_{2} / H$ for the open orbit and hence we may assume $\tilde{D}_{2 n} \subset H$. It follows now that $H=D_{\infty}$. Thus $(G, Y)$ is isomorphic to $\left(\mathrm{SL}_{2}, \mathrm{P}^{2}\right)$ as law chunks of algebraic operation, where $\mathrm{SL}_{2}$ operates on $\mathbf{P}^{2}$ through the irreducible representation of degree 3 (cf. [U3]). Let $g: \mathbf{P}^{2} \ldots \rightarrow Y$ be an $\mathrm{SL}_{2}$-equivariant birational map. By Hironaka, there exists an equivariant blow-ups $g_{1}: Z \rightarrow \mathrm{P}^{2}, g_{2}: Z \rightarrow Y$ such that $g=g_{2} \circ g_{1}^{-1}$. But the orbit decomposition of $\mathbf{P}^{2}$ is $\mathrm{SL}_{2} / D^{\infty} \cup \mathrm{SL}_{2} / B$ and hence $Z=\mathbf{P}^{2}$ and $g$ is a birational morphism hence an isomorphism since $\mathbf{P}^{2}$ is relatively minimal and $Y$ is non-singular.

Theorem (4.7). For $n \geqq 4$, the ordered set $\mathscr{C}(\mathrm{J} 5 ; n)$ contains the unique minimal element which is given by $\left(\mathrm{SL}_{2}, \mathbf{P}\left(E_{n}\right)\right)$ and any other element of $\mathscr{C}(\mathrm{J} 5 ; n)$ is obtained by an equivariant blow-up of the minimal element along curves isomorphic to $\mathbf{P}^{1}$.

Proof. Let $(G, X) \in \mathscr{C}(\mathrm{J} 5 ; n)$. We show that if $\rho(X) \geqq 3$, then we can equivariantly blow down $X$ to a non-singular projective $Y$. In fact, as $X$ is rational, by Mori [Mo] there exists an extremal ray and a morphism $\phi: X \rightarrow Y$ in Theorem (3.1), [Mo]. The 4 cases (3.3.2) . (3.3.5) are already excluded by Lemma (4.4). We have to exclude all the cases in Theorem (3.5) in [Mo].

The case (3.5.1) in [Mo] never happens. Otherwise there would be a 
morphism $(G, X) \rightarrow(G, Y)$ with $Y$ non-singular projective surface. It follows from Lemma (4.11) that $Y \cong \mathbf{P}^{2}$. Now $\rho(X)=\rho(Y)+1=\rho\left(\mathbf{P}^{2}\right)+1$ $=2$, which is a contradiction.

The case (3.5.2) in [Mo] is impossible by Lemma (4.6). The case (3.5.3) is excluded in Section 7, [MU].

We conclude therefore that if $\rho(X) \geqq 3$, then only the case (3.3.1) in [Mo] occurs.

Now we must show that if $(G, X) \in \mathscr{C}(\mathrm{J} 5 ; n)$ and $\rho(X)=2$, then $(G, X)$ is isomorphic to $\left(\mathrm{SL}_{2}, \mathbf{P}\left(E_{n}\right)\right)$. Then as in the $\rho(X) \geqq 3$ case, the extremal ray exists. Since as we have seen in Section $7[\mathrm{MU}] \rho(Y) \geqq 2$ for $(G, Y)$ $\in \mathscr{C}(J 5 ; n)$, the case (3.3.1), [Mo] never happens. The cases (3.3.2) ... (3.3.5), [Mo] are excluded by Lemma (4.4). The case (3.5.2), [Mo] is excluded by Lemma (4.11) and we need not consider the case (3.5.3), [Mo] since $\rho(X)=2$. The only possible case is the (3.5.1), [Mo] and it follows from Lemma (4.4) and Lemma (1.13) that $\varphi: X \rightarrow Y \cong \mathbf{P}^{2}$ is an $\mathrm{SL}_{2}$-equivariant $\mathbf{P}^{1}$-bundle over $\mathbf{P}^{2}$ and hence $X$ is isomorphic to $\mathbf{P}(E)$ for a suitable vector bundle $E$ over $\mathbf{P}^{2}$ of rank 2 since the Brauer group of $\mathbf{P}^{2}$ vanishes. Let $U$ denote the open $\mathrm{SL}_{2}$-orbit on $\mathbf{P}^{2} ; U=\mathbf{P}^{2}$-(the invariant quadric). We show that the orbit decomposition of $\mathbf{P}(E \mid U)$ is $\mathrm{SL}_{2} / \tilde{D}_{2 n}$ and $\mathrm{SL}_{2} / \mathbf{G}_{m}$ and the morphism $\varphi$ makes the latter orbit an étale 2-covering of $U$. In fact as we saw in the proof of Lemma (4.5), the stabilizer at $(0,1,0) \in \mathbf{P}^{2}$ is $\tilde{D}_{\infty}$. $\quad \tilde{D}_{\infty}$ operates on the fibre $\left.\mathbf{P}(E)\right|_{(0,1,0)}$ which contains $\tilde{D}_{\infty} / \tilde{D}_{2 n} \cong \mathbf{P}^{1}-2$ points $=\left\{(a, b) \in \mathbf{P}^{1} \mid a, b \neq 0\right\}=Q$. The operation of $\tilde{D}_{\infty}$ on $Q$ is $(a, b) \mapsto$ $\left(t^{2 n} a, b\right)$ for $\left(\begin{array}{ll}t & 0 \\ 0 & t^{-1}\end{array}\right)$ and $(a, b) \mapsto(b, a)$ for $\left(\begin{array}{ll}-1 & 1\end{array}\right)$. Thus $\tilde{D}_{\infty}$ operates on $\left.\mathbf{P}(E)\right|_{(0,1,0)}$ as on $Q$. Let us put $D^{\prime}=\mathbf{P}(E \mid U)-\mathrm{SL}_{2} / \tilde{D}_{2 n}$. Then $\varphi^{-1}(0,1,0)$ $-Q$ consists of 2 distinct points and these 2 points are interchanged by $\left(\begin{array}{ll}-1 & 1\end{array}\right)$ hence in the same $\mathrm{SL}_{2}$-orbit. Since the morphism $D^{\prime} \rightarrow U$ is $\mathrm{SL}_{2}$-equivariant and surjective, $D^{\prime}$ is an $\mathrm{SL}_{2}$-orbit. Consequently $D^{\prime}$ is isomorphic to $\mathrm{SL}_{2} / \mathbf{G}_{m}$ and $\mathrm{SL}_{2} / \mathbf{G}_{m} \rightarrow \mathrm{SL}_{2} / \tilde{D}_{\infty}$ is of course étale. Now let $D$ be the closure of $D^{\prime}$ in $\mathbf{P}(E)=X$. There exists a line bundle $L$ over $\mathbf{P}^{2}$ such that $\mathcal{O}(D) \cong \mathcal{O}_{\mathbf{P}(E)}(2) \otimes \phi^{*} L$. Hence we have an exact sequence

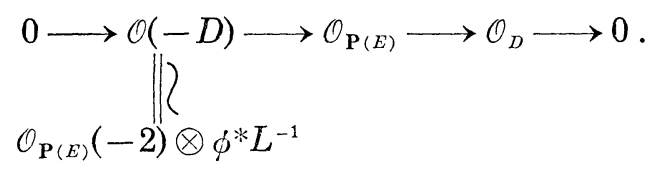

Tensoring $\mathcal{O}_{\mathbf{P}(E)}(1)$ we get 


$$
0 \longrightarrow \mathcal{O}_{\mathbf{P}(E)}(-1) \otimes \phi^{*} L^{-1} \longrightarrow \mathcal{O}_{\mathbf{P}(E)}(1) \longrightarrow \mathcal{O}_{D}(1) \longrightarrow 0 .
$$

If we take the direct image $\phi_{*}$,

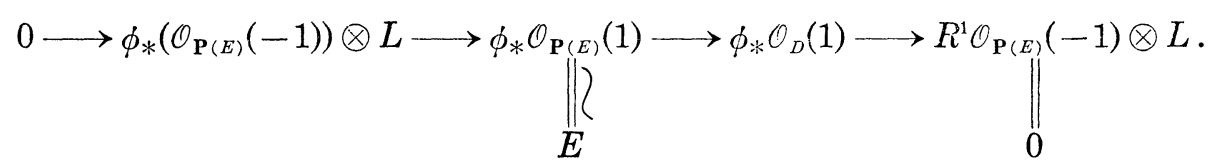

Consequently $E \cong \phi_{*} \mathcal{O}_{D}(1)$. We shall show below that $D \cong \mathbf{P}^{1} \times \mathbf{P}^{1}$. Admitting this for a moment, we conclude that $X=\mathbf{P}(E)$ is isomorphic to $\mathbf{P}\left(E_{i}\right)$ for a some integer $i \geqq 0$. SL $\mathrm{SL}_{2}$ has an open orbit on $\mathbf{P}\left(E_{i}\right)$ isomorphic to $\mathrm{SL}_{2} / \tilde{D}_{2 i}$. Therefore $i=n$ by Theorem (2.1), [U4].

Lemma (4.8). $\quad D$ is isomorphic to $\mathbf{P}^{1} \times \mathbf{P}^{1}$.

Proof. It is sufficient to prove that $D$ is non-singular. In fact if we know that $D$ is non-singular, $D$ is a projective non-singular equivariant completion of $\mathrm{SL}_{2} / \mathbf{G}_{m}$. A particular projective, non-singular equivariant completion of $\mathrm{SL}_{2} / \mathbf{G}_{m}$ is given by $\left(\mathrm{SL}_{2}, \mathbf{P}^{1} \times \mathbf{P}^{1}\right)$, where $\mathrm{SL}_{2}$-operates diagonally. Therefore $\mathbf{P}^{1} \times \mathbf{P}^{1}$ and $D$ are connected by equivariant blowing-ups and downs. But as $\mathrm{SL}_{2}$-has no fixed point on $\mathbf{P}^{1} \times \mathbf{P}^{1}$ and $\mathbf{P}^{1} \times \mathbf{P}^{1}$ is relatively minimal, $\mathbf{P}^{1} \times \mathbf{P}^{1}$ is isomorphic to $D$.

Let us now prove that $D$ is non-singular. Let us denote by $f: \tilde{D} \rightarrow D$ the normalization of $D$ and by $\tilde{L}$ the inverse image $f^{*}\left(\mathcal{O}_{\mathbf{P}(E)}(1) \otimes \mathcal{O}_{D}\right)$. SL operates on $\tilde{D}$ and has no fixed point since $\mathrm{SL}_{2}$ has no fixed point on $D$. Therefore $\tilde{D}$ is a non-singular equivariant completion of $\left(\mathrm{SL}_{2}, \mathrm{SL}_{2} / \mathbf{G}_{m}\right)$ and it follows from the argument above that $\tilde{D}$ is isomorphic to $\mathbf{P}^{1} \times \mathbf{P}^{1}$ with the diagonal action of $\mathrm{SL}_{2}$ and if we put $\varphi^{\prime}=\varphi \mid D, \varphi^{\prime} \circ f: \mathbf{P}^{1} \times \mathbf{P}^{1} \rightarrow \mathbf{P}^{2}$ is the map studied above. Since $f$ is an isomorphism outside the diagonal, $\left(\varphi^{\prime} \circ f\right)_{*} \tilde{L}$ is isomorphic to $E$ when restricted on $\mathbf{P}^{2}-$ (the ramification quadric curve). Therefore $\mathrm{SL}_{2}$ has an open orbit isomorphic to $\mathrm{SL}_{2} / \tilde{D}_{2_{n}}$ on $\mathbf{P}\left(\left(\varphi^{\prime} \circ f\right)_{*} \tilde{L}\right)$ and consequently by Proposition (7) [Sc], and Theorem (2.1), [U4], $\tilde{L} \cong \mathcal{O}_{\mathbf{P}^{1} \times \mathbf{P}^{1}}(a+n, a)$ (or $\mathcal{O}(a, a+n)$ ). This is a contradiction once the following Lemma is proved.

Lemma (4.9). Let $M$ be a line bundle on $D$. If $D$ is singular, then $f^{*} M \cong \mathcal{O}_{\mathbf{P}^{1} \times \mathbf{P}^{1}}(a, a)$.

Proof. The following sublemma is well-known (see [Sc]). To explain the assertion we need 
Notation (4.10). Let $Y$ be a variety, $L$ a line bundle and $\varphi$ a global section of $L^{2}$. Let $L$ be defined by a cocycle $g_{i j}$ for an open covering $\left(U_{i}\right)_{i \in I}$ and assume that $\varphi$ is locally given by $\varphi_{i}$ so that $g_{i j}^{2} \varphi_{i}=\varphi_{j}$. Let $X_{i}=\left\{\left(z_{i}, x_{i}\right) \in \mathbf{A}^{1} \times U_{i} \mid z_{i}^{2}=\varphi_{i}\left(x_{i}\right)\right\}$. Identifying $\left(z_{i}, x_{i}\right) \in \mathbf{A}^{1} \times U_{i}$ and $\left(z_{j}, x_{j}\right)$ $\in \mathbf{A}^{1} \times U_{j}$ when $\left(z_{j}, x_{j}\right)=\left(g_{i j} z_{i}, x_{i}\right)$, we get the $\mathbf{A}^{1}$-bundle $\operatorname{Spec}\left(\oplus_{j \geq 0} L^{\otimes-j}\right)$. Then $X_{i}$ 's are glued together to give $X=\cup X_{i}$. We say that the scheme $X$ is the 2-covering defined by the section $\varphi$ of $L$. The ramification locus of $X \rightarrow Y$ is the 0 -locus of $\varphi$.

Sublemma (4.11). Let $X \rightarrow Y$ be a finite morphism of degree 2, $Y$ being non-singular. If $X$ is Cohen-Macauley, then there exist a line bundle $L$ on $Y$ and a section $\varphi$ of $L^{2}$ such that $X$ is $Y$-isomorphic to the 2-covering of $Y$ defined by $\varphi$.

By Sublemma (4.11), $D$ is defined by a section $s$ of $\mathcal{O}_{\mathbf{p}_{2}}(2 \ell)$ whose reduced 0 -locus is the $\mathrm{SL}_{2}$-invariant quadric. Namely let $h\left(x_{0}, x_{1}, x_{2}\right)$ be the quadric defining the ramification quadric. Then $s=h^{\ell}$. Therefore, as $f \circ \varphi^{\prime}: \mathbf{P}^{1} \times \mathbf{P}^{1} \rightarrow \mathbf{P}^{2}$ is nothing but the canonical map associated with $\mathbf{P}^{1} \times \mathbf{P}^{1} \rightarrow$ (the symmetric product of 2 copies of $\left.\mathbf{P}^{1}\right) \cong \mathbf{P}^{2}, f \circ \varphi^{\prime}$ and $\varphi^{\prime}$ are locally written as follows:

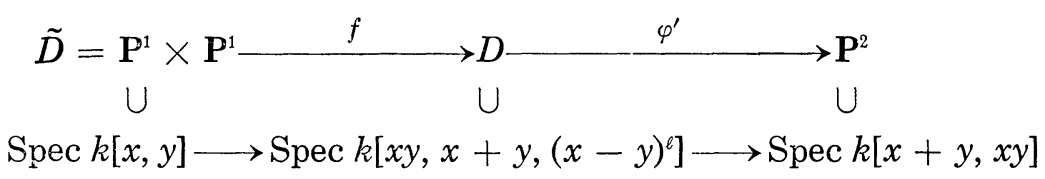

$\ell$ is odd and $\geqq 3$ since $D$ is an irreducible 2-covering of $\mathbf{P}^{2}$ and singular. Let $\Delta(2)$ denote the non-reduced subscheme of $\mathbf{P}^{1} \times \mathbf{P}^{1}$ defined by $I_{\Delta}^{2}$, where $I_{\Delta}$ is the defining ideal of the diagonal. The inclusion morphism $L: \Delta(2)$ $\rightarrow \mathbf{P}^{1} \times \mathbf{P}^{1}$ is locally written by $k[x, y] /(x-\mathrm{y})^{2} \leftarrow k[x, y]$. Composing with $f$, we get $\psi: \Delta(2) \rightarrow D$ which is locally given by $k[x, y] /(x-y)^{2} \leftarrow k[x+y$, $\left.x y,(x-y)^{\ell}\right]$. Let now $i$ be the automorphism of $\mathbf{P}^{1} \times \mathbf{P}^{1}$ interchanging factors. It follows from the local expression that $f \circ i \circ L=\psi$. Therefore if $f^{*} M$ $=\mathcal{O}_{\mathbf{P} 1 \times \mathbf{P} 1}(a, b)$, then $L^{*} \mathcal{O}(a, b) \cong L^{*} \mathcal{O}(b, a)$. Now the Lemma follows from

Lemma (4.12). If $\mathcal{O}_{\Delta(2)} \otimes \mathcal{O}(a, b) \cong \mathcal{O}_{\Delta(2)} \otimes \mathcal{O}(b, a)$, then $a=b$.

Proof. By tensoring $\mathcal{O}(-b,-a)$, we have to show that $\mathcal{O}_{\Delta(2)} \otimes \mathcal{O}(j,-j)$ is not isomorphic to $\mathcal{O}_{\Delta(2)}$ if $j \geqq 1$. In fact we have an exact sequence:

$$
0 \longrightarrow \mathcal{O}(-2,-2) \longrightarrow \mathcal{O}_{\mathbf{P}^{1} \times \mathbf{P}^{1}} \longrightarrow \mathcal{O}_{\Delta(2)} \longrightarrow 0 \text {. }
$$


Assume that $\mathcal{O}_{\Delta(2)} \otimes \mathcal{O}(j,-j) \cong \mathcal{O}_{\Delta(2)}$ with $j \geqq 1$. Tensoring $\mathcal{O}(j,-j)$ with the exact sequence (4.13), we get

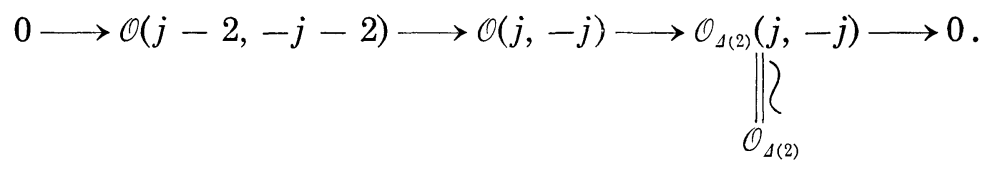

Since the morphisms in this exact sequence are $\mathrm{SL}_{2}$-morphisms, we get $\mathrm{SL}_{2}$-exact sequence

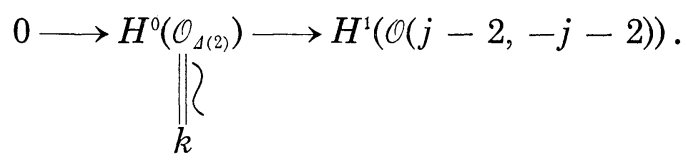

The $\mathrm{SL}_{2}$-module $H^{1}(\mathcal{O}(j-2,-j-2))$ contains the trivial module $k$. Let $W_{d}$ denote the irreducible $\mathrm{SL}_{2}$-module of degree $d+1$ if $d \geqq 0$ and $W_{d}=0$ if $d<0$. By the Künneth formula and the Serre duality, $H^{1}(\mathcal{O}(j-2, j-2))$ $\cong W_{j-2} \otimes W_{j}$ for $j \geqq 1$. By the Clebsch-Gordan formula (p. 126, [Hu]) $W_{j-2} \otimes W_{j} \cong W_{2 j-2} \oplus W_{2 j-4} \oplus \cdots \oplus W_{2}, W_{j-2} \otimes W_{j}$ contains no trivial $\mathrm{SL}_{2}$ module $k$ which is absurd.

\section{§5. Equivariant completions of J6}

We have to consider the operation $\left(G, W_{m, n}\right)=\left(\mathbf{G}_{m} \times \mathrm{SL}_{2} \times \mathrm{SL}_{2}, \mathbf{G}_{m} \times\right.$ $\left.\mathrm{SL}_{2} \times \mathrm{SL}_{2} / H_{m, n}\right)$, where

$$
H_{m, n}=\left\{\left(t_{1}^{m} t_{2}^{n},\left(\begin{array}{ll}
t_{1} & x \\
0 & t_{1}^{-1}
\end{array}\right),\left(\begin{array}{ll}
t_{2} & y \\
0 & t_{2}^{-1}
\end{array}\right)\right) \in \mathbf{G}_{m} \times \mathrm{SL}_{2} \times \mathrm{SL}_{2} \mid t_{1}, t_{2} \in k^{*}, x, y \in k\right\}
$$

and $m, n$ are integers with $m \geqq 2,-2 \geqq n$. We denote $\mathscr{C}\left(G, W_{m, n}\right)$ by $\mathscr{C}(\mathrm{J} 6 ; m, n) . \quad W_{m, n}$ is the principal $\mathbf{G}_{m}$-bundle of bidegree $(m, n)$ over $\mathrm{SL}_{2} / B \times \mathrm{SL}_{2} / B \cong \mathbf{P}^{1} \times \mathbf{P}^{1}$. Hence $\left(G, W_{m, n}\right)$ has a natural equivariant completion $L_{m, n}=\mathbf{P}\left(\mathcal{O}_{\mathbf{P} 1 \times \mathbf{P} 1} \oplus \mathcal{O}_{\mathbf{P} 1 \times \mathbf{P} 1}(-m,-n)\right)$ and we know that we have a G-equivariant morphism $(\varphi, f):\left(G, \mathbf{P}\left(\mathcal{O}_{\mathbf{P}^{1} \times \mathbf{P}_{1}} \oplus \mathcal{O}_{\mathbf{P}^{1} \times \mathbf{P}}(m, n)\right) \rightarrow\left(\mathrm{SL}_{2} \times \mathrm{SL}_{2}\right.\right.$, $\left.\mathbf{P}^{1} \times \mathbf{P}^{1}\right), \varphi$ being the projection $([\mathrm{U} 4])$. We can regard $\operatorname{Spec}\left(\oplus_{\ell \geq 0} \mathcal{O}(-\ell m\right.$, $-\ell n))$ as a Zariski open set in $\mathbf{P}\left(\mathcal{O}_{\mathbf{P}^{1} \times \mathbf{P}^{1}} \oplus \mathcal{O}_{\mathbf{P}^{1} \times \mathbf{P}^{1}}(-m,-n)\right)$. We set

$$
\left.D_{\infty}=\mathbf{P}\left(\mathcal{O}_{\mathbf{P} 1 \times \mathbf{P} 1} \oplus \mathcal{O}_{\mathbf{P} 1 \times \mathbf{P} 1}(-m,-n)\right)-\operatorname{Spec} \bigoplus_{\ell \geq 0} \mathcal{O}(-\ell m, \ell n)\right)
$$

and $D_{0}=\operatorname{Spec}\left(\oplus_{\ell \geq 0}(-\ell m, \ell n)\right)-W_{m, n}$ (=the zero section of the line bundle Spec $\left(\oplus_{\ell \geq 0} \mathcal{O}(-\ell m, \ell n)\right)$. Namely adding the 0 -section $D_{0}$ to the $\mathbf{G}_{m}$-bundle $W_{m, n}$, we get the line bundle $\operatorname{Spec} \mathcal{O}\left(\oplus_{\ell \geqq 0}(-\ell m,-\ell n)\right)$ and further- 
more by adding the $\infty$-section $D_{\infty}$ to the line bundle Spec $\left(\oplus_{\ell \geqq 0} \mathcal{O}(-\ell m\right.$, $-\ell n)$ ), we get the $\mathbf{P}^{1}$-bundle $\mathbf{P}(\mathcal{O} \oplus \mathcal{O}(-m,-n))$ over $\mathbf{P}^{1} \times \mathbf{P}^{1}$. By the equivariant morphism $f$, the orbit decomposition of $\mathbf{P}(\mathcal{O} \oplus \mathcal{O}(-m,-n))$ is $W_{m, n} \cup D_{0} \cup D_{\infty}$.

Theorem (5.1). $\mathscr{C}(\mathrm{J} 6 ; m, n)(m \geqq 2,-2 \geqq n)$ consists of a single element $\left(G, L_{m, n}\right)$.

Proof. Let $(G, X) \in \mathscr{C}(\mathrm{J} 6 ; m, n)$. By Hironaka's Theorem, there exists an equivariant blow-up $f_{1}: Y \rightarrow \mathbf{P}(\mathcal{O} \oplus \mathcal{O}(-m,-n))$ and a birational morphism $f_{2}: Y \rightarrow X$. It follows from orbit decomposition that $f_{1}$ is an isomorphism. Thus we get a birational morphism $f_{2}: \mathbf{P}(\mathcal{O} \oplus \mathcal{O}(-m,-n)) \rightarrow$ $X$. We have $\mathcal{O}\left(-D_{0}\right) \otimes \mathcal{O}_{D_{0}} \cong \mathcal{O}_{\mathbf{P} 1 \times \mathbf{P} 1}(m, n)$ and $\mathcal{O}\left(-D_{\infty}\right) \otimes \mathcal{O}_{D_{\infty}} \cong \mathcal{O}_{\mathbf{P} 1 \times \mathbf{P} 1}(-m$, $-n)$ if we identify $D_{0}$ and $D_{\infty}$ with $\mathbf{P}^{1} \times \mathbf{P}^{1}$ by the equivariant map $f$. It follows from Lemma (1.12) that neither $f_{2}\left(D_{0}\right)$ nor $f_{2}\left(D_{\infty}\right)$ is reduced to a point. Since $m \geqq 2,-2 \geqq n$, then we can blow down neither $D_{0}$ nor $D_{\infty}$ to a curve (cf. Theorem (1.7)). Hence by Zariski's Main Theorem $f_{2}$ is an isomorphism.

\section{§6. Equivariant completions of J7}

$\mathrm{J}_{m}^{\prime}$ is by definition $\operatorname{Spec}\left(S\left(\mathcal{O}_{\mathbf{P} 2}(-m)\right)\right.$; the total space of the line bundle of degree $m$ over $\mathbf{P}^{2}$. We study the operation (J7) (Aut $\left.{ }^{0} \mathrm{~J}_{m}^{\prime}, \mathrm{J}_{m}^{\prime}\right)(m \geqq 2)$. We know by Proposition (4.8), [U4] Aut ${ }^{0} \mathrm{~J}_{m}^{\prime}$ respects the fibration $\mathrm{J}_{m} \rightarrow \mathbf{P}^{2}$. Therefore the operation (J7) (Aut ${ }^{0} \mathrm{~J}_{m}^{\prime}, \mathrm{J}_{m}^{\prime}$ ) has a natural equivariant completion (Aut ${ }^{0} \mathrm{~J}_{m}, \mathrm{~J}_{m}$ ) by Corollary (4.10), [U4] where $\mathrm{J}_{m} \operatorname{denotes} \mathbf{P}\left(\mathcal{O}_{\mathbf{P}^{2}} \oplus\right.$ $\left.\mathcal{O}_{\mathrm{P}_{2}}(-m)\right)$. We denote $\mathscr{C}\left(\right.$ Aut $\left.^{0} \mathrm{~J}_{m}^{\prime}, \mathrm{J}_{m}\right)$ by $\mathscr{C}(\mathrm{J} 7 ; m)$.

Theorem $(6.1) . \quad \mathscr{C}(\mathrm{J} 7 ; m)(m \geqq 2)$ consists of a single element (Aut ${ }^{0} J_{m}$, $\left.\mathrm{J}_{m}\right)$.

Proof. For the same reason as in the case (J6), it is sufficient to notice that we can not collapse the orbit $D_{\infty}=\mathrm{J}_{m}-\mathrm{J}_{m}^{\prime} \cong \mathbf{P}^{2}$ to a smooth point. In fact, let $f: \mathrm{J}_{m} \rightarrow X$ be a birational morphism such that $X$ is non-singular and projective, $f$ is biregular outside $D_{\infty}$ and such that $f\left(D_{\infty}\right)$ is a point. Let $H=\pi^{*} \mathcal{O}_{\mathbf{P}^{2}}(1)$ so that $H \otimes \mathcal{O}_{D_{\infty}} \cong \mathcal{O}_{\mathbf{P}^{2}}(1)$, where $\pi: \mathrm{J}_{m} \rightarrow \mathbf{P}^{2}$ is the projection. Then $\mathcal{O}_{D_{\infty}} \cong f *\left(f_{*} H\right) \otimes \mathcal{O}_{D_{\infty}} \cong\left(H \otimes \mathcal{O}\left(r D_{\infty}\right)\right) \otimes \mathcal{O}_{D_{\infty}} \cong$ $\mathcal{O}_{\mathbf{P}^{2}}(1) \otimes\left(\mathcal{O}\left(r D_{\infty}\right) \otimes \mathcal{O}_{D_{\infty}}\right)$. Hence $r=1$ and $\mathcal{O}\left(D_{\infty}\right) \otimes \mathcal{O}_{D_{\infty}} \cong \mathcal{O}_{\mathbf{P}^{2}}(-1)$. But $\mathcal{O}\left(D_{\infty}\right) \otimes \mathcal{O}_{D_{\infty}} \cong \mathcal{O}_{\mathbf{P}}(-m)$, which is a contradiction. 


\section{§ 7. Equivariant completions of J8}

We study (J8) (Aut $\left.{ }^{0} L_{m, n}^{\prime}, L_{m, n}^{\prime}\right)(m \geqq n \geqq 1): L_{m, n}^{\prime}$ is the total space $\operatorname{Spec}(S(\mathcal{O}(-m) \otimes \mathcal{O}(-n)))$ of the line bundle $\mathcal{O}(m, n)$ of bidegree $(m, n)$ over $\mathbf{P}^{1} \times \mathbf{P}^{1}$. The operation (J8) (Aut ${ }^{0} L_{m, n}^{\prime}, L_{m, n}^{\prime}$ ) respects the fibration $L_{m, n}^{\prime} \rightarrow$ $\mathbf{P}^{1} \times \mathbf{P}^{1}$ by Proposition (4.11) and hence by Corollary (4.12) the operation (J8) (Aut ${ }^{0} L_{m, n}^{\prime}, L_{m, n}^{\prime}$ ) has a natural equivariant completion (Aut ${ }^{0} L_{m, n}, L_{m, n}$ ), where $L_{m, n}$ denotes the $\mathbf{P}^{1}$-bundle $\mathbf{P}(\mathcal{O} \oplus \mathcal{O}(-m,-n))$ over $\mathbf{P}^{1} \times \mathbf{P}^{1}$. If $n=1$, by the composite morphism $L_{m, 1} \stackrel{\pi}{\rightarrow} \mathbf{P}^{1} \times \mathbf{P}^{1} \stackrel{p_{1}}{\rightarrow} \mathbf{P}^{1}$, we can regard $L_{m, 1}$ equivariantly as $F_{1}$-bundle over $\mathbf{P}^{1}$. Replacing each fibre $F_{1}$ by $\mathbf{P}^{2}$, we get an equivariant completion (Aut ${ }^{0} L_{m, 1}^{\prime}, X_{m}$ ). The $\mathbf{P}^{2}$-bundle $X_{m}$ over $\mathbf{P}^{1}$ is isomorphic to $\mathbf{P}\left(\mathcal{O}_{\mathbf{P} 1} \oplus \mathcal{O}_{\mathbf{P}_{1}} \oplus \mathcal{O}_{\mathbf{P}_{1}}(-m)\right)$ by its construction. We denote $\mathscr{C}\left(\right.$ Aut $\left.^{0} L_{m, n}^{\prime}, L_{m, n}^{\prime}\right)$ by $(\mathrm{J} 8 ; m, n)$.

TheOREM (7.1). If $m \geqq n \geqq 2, \mathscr{C}(\mathrm{J} 8 ; m, n)$ consists of one element (Aut $^{0}$ $\left.L_{m, n}, L_{m, n}\right)$. The ordered set $\mathscr{C}(\mathrm{J} 8 ; m, 1)$ consists of 2 elements; (Aut ${ }^{0} L_{m, 1}^{\prime}$, $\left.L_{m, 1}\right)>\left(\right.$ Aut $\left.^{0} L_{m, 1}^{\prime}, X_{m}\right)(m \geqq 1)$.

Theorem is proved by the same method as Theorem (6.1). Hence we omit the proof.

\section{§ 8. Equivariant completions of J9}

Let $C_{\infty}=\mathrm{F}_{n}-\mathrm{F}_{n}^{\prime}$; namely $C_{\infty}$ is the section of $\mathbf{P}^{1}$-bundle $f_{n}: \mathrm{F}_{n} \rightarrow \mathbf{P}^{1}$ with $C_{\infty}^{2}=-n$. The section with this property is uniquely determined and called the infinity section of $\mathrm{F}_{n}$ (see (1.2)). We denote by $\mathcal{O}_{\mathrm{F}_{n}}(t)$ the line bundle $f_{n}^{*} \mathcal{O}_{\mathbf{P} 1}(t)$ and by $\mathcal{O}_{\mathrm{F}_{n}^{\prime}}(t)$ its restriction on $\mathrm{F}_{n}^{\prime}$.

The variety $\mathrm{F}_{m, n}^{\prime}(m \geqq n \geqq 1)$ is the total space of the vector bundle $\mathcal{O}_{\mathbf{P}_{1}}(m) \oplus \mathcal{O}_{\mathbf{P}_{1}}(n)$ over $\mathbf{P}^{1}: \mathrm{F}_{m, n}^{\prime}=\operatorname{Spec}\left(S\left(\mathcal{O}_{\mathbf{P}_{1}}(-m) \oplus \mathcal{O}_{\mathbf{P}_{1}}(-n)\right)\right)$, where $S(E)$ denotes the symmetric algebra on $E$. We can regard $\mathrm{F}_{m n}^{\prime}$ also as the total space of a line bundle $\mathcal{O}_{\mathrm{F}_{n}^{\prime}}(m)$ over $\mathrm{F}_{n}^{\prime}$. Namely $\mathrm{F}_{m, n}^{\prime}=$ $\operatorname{Spec}\left(S\left(\mathcal{O}_{\mathrm{F}_{n}^{\prime}}(-m)\right)\right)$ and we have a morphism $\mathrm{F}_{m, n}^{\prime} \rightarrow \mathrm{F}_{n}^{\prime}$ giving the bundle structure over $\mathrm{F}_{n}^{\prime}$. (J9) is the operation (Aut $\left.{ }^{0} \mathrm{~F}_{m, n}^{\prime}, \mathrm{F}_{m, n}^{\prime}\right)(m>n \geqq 2$ ). Since (Aut ${ }^{0} \mathrm{~F}_{m, n}^{\prime}, \mathrm{F}_{m, n}^{\prime}$ ) respects the fibration by Proposition (4.13), [U4], to complete equivariantly the $\mathbf{A}^{1}$-bundle $\mathbf{F}_{m, n}^{\prime}$ over $\mathbf{F}_{n}^{\prime}$, first we want to equivariantly extend it to an $\mathbf{A}^{1}$-bundle over $\mathrm{F}_{n}$. For this purpose, in view of Lemma (1.9) in section 1 of this paper, Lemma (4.4), Proposition (4.13) and Corollary (4.17) all in [U4], it is sufficient to find a line bundle $M$ over $\mathrm{F}_{n}$ such that (1) $M$ is Aut $^{0} \mathrm{~F}_{n}$-equivariant, $M$ is isomorphic to $\mathcal{O}_{F_{n}^{\prime}}(m)$ 
when restricted over $\mathrm{F}_{n}^{\prime}$ and such that (2) the restriction $H^{\circ}\left(\mathrm{F}_{n}, M\right)$ $\rightarrow H^{0}\left(\mathrm{~F}_{n}^{\prime}, M\right) \cong H^{0}\left(\mathrm{~F}_{n}^{\prime}, \mathcal{O}_{\mathrm{F}_{n}^{\prime}}(m)\right)$ induces an isomorphism. In fact, then $\operatorname{Spec}\left(S\left(M^{-1}\right)\right) \supset \operatorname{Spec}\left(S\left(\mathcal{O}_{\mathrm{F}_{n}^{\prime}}(-m)\right)\right.$ is a desired extension to $\mathrm{F}_{n}$ and $\mathbf{P}\left(\mathcal{O}_{\mathrm{F}_{n}} \oplus\right.$ $\left.M^{-1}\right)$, which is an equivariant completion of $\operatorname{Spec}\left(S\left(M^{-1}\right)\right)$, is an equivariant completion of $\left(\right.$ Aut $\left.^{0} \mathrm{~F}_{m, n}^{\prime}, \mathrm{F}_{m, n}^{\prime}\right)$.

We choose an integer $\ell$ such that $m=\ell n+r, 0 \leqq r<n$ (cf. Corollary (4.17), [U4]).

Lemma (8.1). (Aut ${ }^{0} \mathrm{~F}_{m, n}^{\prime}, \mathrm{F}_{m, n}^{\prime}$ ) has an equivariant completion (Aut ${ }^{0} \mathrm{~F}_{m, n}^{k}$, $\left.\mathrm{F}_{m, n}^{k}\right)$ for any integer $k \geqq \ell$, where $\mathrm{F}_{m, n}^{k}$ denotes the $\mathbf{P}^{1}$-bundle $f_{m, n}^{k}: \mathbf{P}\left(\mathcal{O}_{\mathrm{F}_{n}} \oplus\right.$ $\left.\mathcal{O}_{\mathrm{F}_{n}}(-m) \otimes \mathcal{O}_{\mathrm{F}_{n}}\left(-k C_{\infty}\right)\right) \rightarrow \mathrm{F}_{n}$.

Proof. By the argument preceding the Lemma, we look for a condition for the restriction $0 \rightarrow H^{\circ}\left(\mathrm{F}_{n}, \mathcal{O}_{\mathrm{F}_{n}}(m) \otimes \mathcal{O}_{\mathrm{F}_{n}}\left(k C_{\infty}\right)\right) \rightarrow H^{0}\left(\mathrm{~F}_{n}^{\prime}, \mathcal{O}_{\mathrm{F}_{n}^{\prime}}(m)\right)$ to be isomorphism. It follows from the spectral sequences in (1.2), (1.2.1) and (1.3.1),

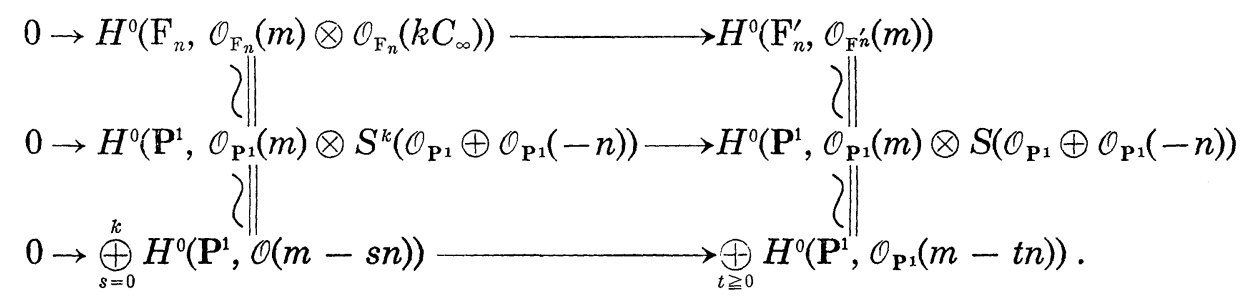

Therefore $k \geqq \ell$ is the necessary and sufficient condition.

We denote by $D_{2}^{k}$ the $\infty$-section of the $\mathbf{P}^{1}$-bundle $\mathrm{F}_{m, n}^{k}$ over $\mathrm{F}_{n}$ so that $D_{2}^{k}=\mathrm{F}_{m, n}^{k}-\operatorname{Spec}\left(S\left(\mathcal{O}_{F_{n}}(-m)\right) \otimes \mathcal{O}_{F_{n}}\left(-k C_{\infty}\right)\right)$ and by $D_{1}^{k}$ the inverse image $\left(f_{m, n}^{k}\right)^{-1} C_{\infty}$. We have a morphism

$\left(\mathrm{Id}, f_{m, n}^{k}\right):\left(\right.$ Aut $\left.^{0} \mathrm{~F}_{m, n}^{k}, \mathrm{~F}_{m, n}^{k}\right) \longrightarrow\left(\right.$ Aut $\left.^{0} \mathrm{~F}_{m, n}^{k}, \mathrm{~F}_{n}\right)$ of algebraic operations.

The projection $f_{m, n}^{k}$ induces an isomorphism $D_{2}^{k} \cong \mathrm{F}_{n}$ and the divisor $D_{1}^{k}$ is isomorphic to $\mathbf{P}\left(\left(\mathcal{O} \oplus \mathcal{O}(-m) \otimes \mathcal{O}\left(-k C_{\infty}\right)\right) \otimes \mathcal{O}_{C_{\infty}}\right)$ hence to $\mathbf{P}\left(\mathcal{O}_{\mathbf{P}_{1}} \oplus\right.$ $\left.\mathcal{O}_{\mathbf{P}_{\mathrm{r}}}(-m+k n)\right) \cong \mathrm{F}_{|-m+k n|}$. The intersection $D_{2}^{k} \cap D_{1}^{k}$ is the $\infty$-section of the ruled surface $D_{1}^{k} \cong \mathrm{F}_{-m+k n}$ (see Fig (8.2)).

We use the orbit decomposition of $\mathrm{F}_{m, n}^{k}$ in a substantial way.

Lemma (8.3). The orbit decomposition of $\left(\right.$ Aut $^{0} \mathrm{~F}_{m, n}^{\prime}, \mathrm{F}_{m, n}^{e}$ ) is $\mathrm{F}_{m, n}^{\prime} U$ $\left(D_{1}^{\ell}-D_{1}^{\ell} \cap D_{2}^{\ell}\right) \cup\left(D_{2}^{\ell}-D_{1}^{\ell} \cap D_{2}^{\ell}\right) \cup\left(D_{1}^{\ell} \cap D_{2}^{\ell}\right)$. For any integer $k>\ell$, the orbit decomposition of $\left(\right.$ Aut $\left.^{0} \mathrm{~F}_{m, n}^{\prime}, \mathrm{F}_{m, n}^{k}\right)$ is $\mathrm{F}_{m}^{\prime} \cup\left(D_{1}^{k}-D_{1}^{k} \cap D_{2}^{k}-C\right) \cup\left(D_{2}^{k}-D_{1}^{k} \cap D_{2}^{k}\right)$ $\cup\left(D_{1}^{k} \cap D_{2}^{k}\right) \cup C$, where $C$ is a 0 -section of the ruled surface $D_{1}^{k} \cong \mathrm{F}_{-m+n k}$. 
Fig. (8.2)

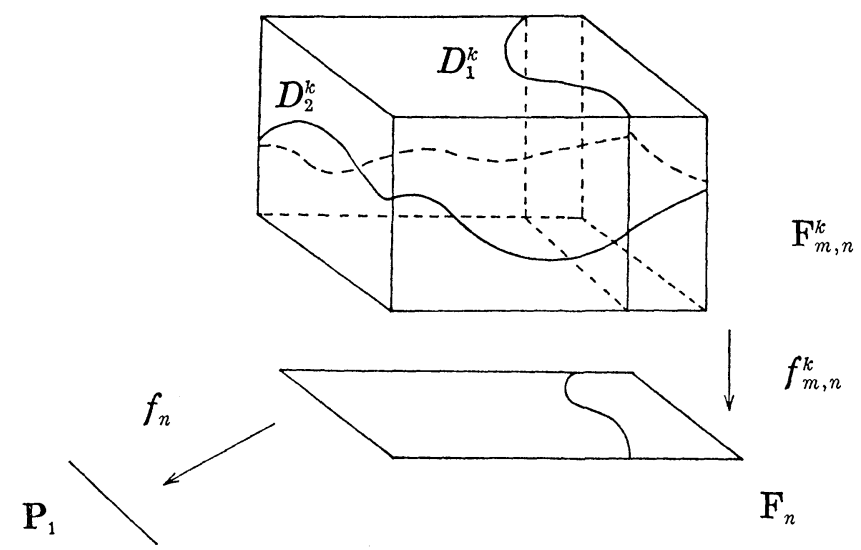

Proof. It follows from Corollary (4.17) and Lemma (4.4), [U4] that $\mathrm{F}_{m, n}^{\prime}, D_{1}^{j}$ and $D_{2}^{j}, D_{1}^{j} \cap D_{2}^{j}$ are Aut $\mathrm{F}_{m, n}^{\prime}$-invariant subvarieties of $\mathrm{F}_{m, n}^{j}$ for $j \geqq \ell$. By the morphism $f_{m, n}^{j}$, the invariant divisor $D_{2}^{j}$ decomposes into the union $\left(D_{2}^{j}-D_{1}^{j} \cap D_{2}^{j}\right) \cup\left(D_{1}^{j} \cap D_{2}^{j}\right)$ of 2 invariant subvarieties. It follows from Corollary (4.17), [U4] that the unipotent part of the $\operatorname{Ker}\left(\right.$ Aut $^{0} \mathrm{~F}_{m, n}^{\prime} \rightarrow$ Aut $\left.\mathrm{F}_{n}^{\prime}\right)$ is $H^{0}\left(\mathrm{~F}_{n}^{\prime}, \mathcal{O}_{\mathrm{F}_{n}^{\prime}}(m)\right)$. In the Proof of Lemma (8.1) we choose $j \geqq \ell$ so that the restriction

$\left(^{*}\right) \quad H^{0}\left(\mathrm{~F}_{m}, \mathcal{O}_{\mathrm{F}_{n}}(m) \otimes \mathcal{O}_{\mathrm{F}_{n}}\left(j C_{\infty}\right)\right) \longrightarrow H^{0}\left(\mathrm{~F}_{n}^{\prime}, \mathcal{O}_{\mathrm{F}_{n}}(m) \otimes \mathcal{O}_{\mathrm{F}_{n}}\left(j C_{\infty}\right)\right)=H^{0}\left(\mathrm{~F}_{n}^{\prime}, \mathcal{O}_{\mathrm{F}_{n}^{\prime}}(m)\right)$

induces an isomorphism between the cohomology groups and $l$ is the smallest among such integers. Now the injection $\mathcal{O}_{\mathrm{F}_{n}}(m) \otimes \mathcal{O}_{\mathrm{F}_{n}}\left(j C_{\infty}\right) \rightarrow$ $\mathcal{O}_{\mathrm{F}_{n}}(m) \otimes \mathcal{O}_{\mathrm{F}_{n}}\left((j+1) C_{\infty}\right)$ induces an injection $0 \rightarrow H^{0}\left(F_{n}, \mathcal{O}_{\mathrm{F}_{n}}(m) \otimes \mathcal{O}_{\mathrm{F}_{n}}\left(j C_{\infty}\right)\right) \rightarrow$ $H^{0}\left(\mathrm{~F}_{n}, \mathcal{O}_{\mathrm{F}_{n}}(m) \otimes \mathcal{O}_{\mathrm{F}_{n}}\left((j+1) C_{\infty}\right)\right)$, which is thus an isomorphism since their dimensions coincide by $\left(^{*}\right)$. Therefore all the sections of $H^{0}\left(\mathrm{~F}_{n}, \mathcal{O}_{\mathrm{F}_{n}}(m)\right.$ $\left.\otimes \mathcal{O}_{F_{n}}(j+1) C_{\infty}\right)$ vanishes on $C_{\infty}$. It follows from Lemma (1.9) that $H^{0}\left(\mathrm{~F}_{n}, \mathcal{O}_{\mathrm{F}_{n}}(m) \otimes \mathcal{O}_{\mathrm{F}_{n}}\left(j C_{\infty}\right)\right)$ operates on $D_{1}^{j}$ trivially if $j>\ell$ and non-trivially on $D_{1}^{\ell}$. The Lemma now follows from Lemma (1.8) and from [U3].

Definition (8.4). Let $X$ be a projective non-singular threefold and $S$ a finite set of irreducible divisors $D_{i}$ on $X(1 \leqq i \leqq n)$ such that (1) each $D_{i}$ is isomorphic to a rational ruled surface $\mathrm{F}_{m_{i}}, m_{i} \geqq 0(1 \leqq i \leqq n)$, (2) if $D_{i} \cap D_{j} \neq \varnothing$, then $D_{i}$ and $D_{j}$ intersects transversely along $D_{i} \cap D_{j}=$ $C_{i j}\left(\cong \mathbf{P}^{1}\right)$ which is a section of the rational ruled surfaces $\mathrm{F}_{m_{i}}$ and $\mathrm{F}_{m_{j}}$ with $\left|\left(C_{i j} \cdot C_{i j}\right)_{D_{i}}\right|=m_{i}$ and $\left|\left(C_{i j} \cdot C_{i j}\right)_{D_{j}}\right|=m_{j}$ and such that (3) for any three distinct divisors $D_{a}, D_{b}, D_{c} \in S, D_{a} \cap D_{b} \cap D_{c}=\varnothing$. We associate to 
$S$ a diagram $D . \quad D$ consists of $n$ segments $\ell_{1}, \ell_{2}, \cdots, \ell_{n}$ on the plane satisfying the following conditions.

(a) $\ell_{i} \cap \ell_{j}$ is either empty or a point for any $1 \leqq i<j \leqq n$.

(b) $\ell_{i} \cap \ell_{j} \neq \varnothing$ if and only if $D_{i} \cap D_{j} \neq \varnothing$.

EXAMPLES (8.5.1). We taking for $S$ the set of $G$-invariant divisors on $\mathrm{F}_{m, n}^{k}$, it follows from Lemma (8.3) that $S$ is described by

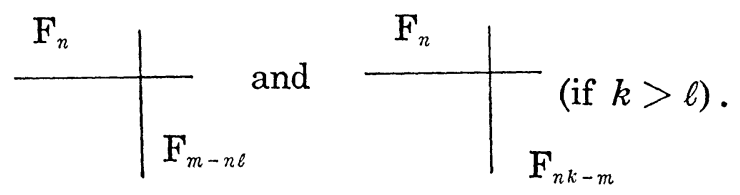

Assuming $m-n \ell>0$, to indicate that $C^{\prime}=\mathrm{F}_{n} \cap \mathrm{F}_{m-n \ell}$ has the intersection numbers; $\left(C^{\prime 2}\right)_{\mathrm{F}_{m-n \ell}}=n \ell-m<0,\left(C^{\prime 2}\right)_{\mathrm{F}_{n}}=-n<0$, we complete the diagram by making the segments into arrows:

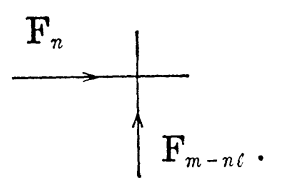

For $k$ with $n k-m>0, C^{\prime}=\mathrm{F}_{n} \cap \mathrm{F}_{n k-m}$ has the intersection number $\left(C^{\prime 2}\right)_{\mathrm{F}_{n}}=-n<0,\left(C^{\prime 2}\right)_{\mathrm{F}_{n k-m}}=n k-m>0$. Therefore our diagram is

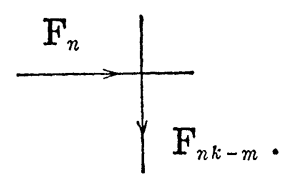

(8.5.2). The diagram

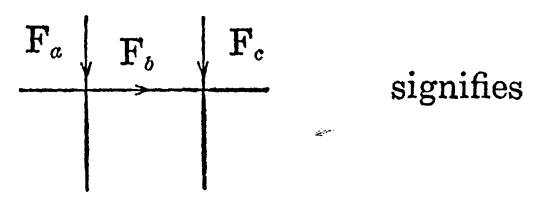

$\mathrm{F}_{a}$ and $\mathrm{F}_{b}$ (resp. $\mathrm{F}_{b}$ and $\mathrm{F}_{c}$ ) intersect transversely along sections of the ruled surfaces and we have the intersection numbers

$$
\left(\mathrm{F}_{a} \cap \mathrm{F}_{a}\right)_{\mathrm{F}_{a}}^{2}=-a,\left(\mathrm{~F}_{a} \cap \mathrm{F}_{b}\right)_{\mathrm{F}_{b}}^{2}=b,\left(\mathrm{~F}_{a} \cap \mathrm{F}_{c}\right)_{\mathrm{F}_{b}}^{2}=-b
$$

and $\left(\mathrm{F}_{b} \cap \mathrm{F}_{c}\right)_{\mathrm{F}_{c}}^{2}=-c$. 
(8.5.3). The diagram

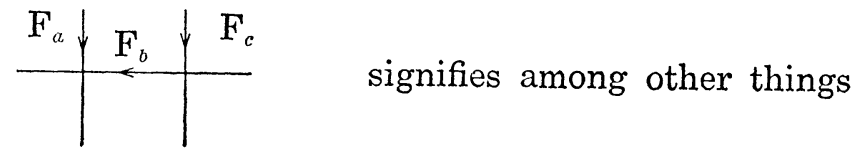

$$
\left(\mathrm{F}_{a} \cap \mathrm{F}_{b}\right)_{\mathrm{F}_{a}}^{2}=-a,\left(\mathrm{~F}_{a} \cap \mathrm{F}_{b}\right)_{\mathrm{F}_{b}}^{2}=-b,\left(\mathrm{~F}_{b} \cap \mathrm{F}_{c}\right)_{\mathrm{F}_{b}}^{2}=b \text { and }\left(\mathrm{F}_{b} \cap \mathrm{F}_{c}\right)_{\mathrm{F}_{c}}^{2}=-c \text {. }
$$

The following Lemma shows the convenience of the diagram.

LEMma (8.5.4). Let $X$ be a non-singular projective threefold.

(i) Assume that we have two divisors on $X$ isomorphic respectively to $\mathrm{F}_{a}$ and $\mathrm{F}_{b}$ such that they are expressed in the diagram

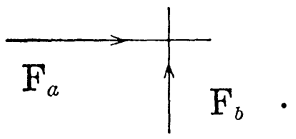

Let $p: Y \rightarrow X$ be $a$ blow-up of $X$ along $\mathrm{F}_{a} \cap \mathrm{F}_{b}$. If $a>b \geq 1$, then the divisor $p^{-1}\left(\mathrm{~F}_{a} \cup \mathrm{F}_{b}\right)$ on $Y$ is expressed in terms of the diagram

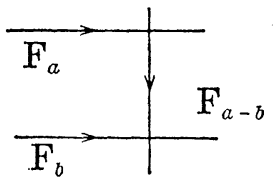

where $\mathrm{F}_{a}$ and $\mathrm{F}_{b}$ are the proper transforms and $\mathrm{F}_{a-b}$ is the exceptional divisor.

(ii) Assume that we have two divisors on $X$ isomorphic to $\mathrm{F}_{a}$ and $\mathrm{F}_{b}$ such that they are expressed in the diagram

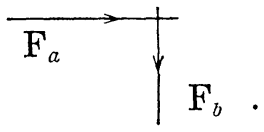

Let $p: Y \rightarrow X$ be a blow up of $X$ along $\mathrm{F}_{n} \cap \mathrm{F}_{b}$. If $a, b \geq 1$, then the divisor $p^{-1}\left(\mathrm{~F}_{a} \cup \mathrm{F}_{b}\right)$ on $Y$ is expressed in terms of the diagram

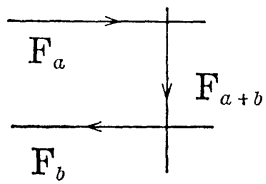


where $\mathrm{F}_{a}$ and $\mathrm{F}_{b}$ are the proper transforms and $\mathrm{F}_{a+b}$ is the exceptional divisor for $p$.

Proof. Since $\mathrm{F}_{a}$ and $\mathrm{F}_{b}$ intersect transversely, the exceptional divisor $E$ is a rational ruled surface and $E \cap \mathrm{F}_{a}, E \cap \mathrm{F}_{b}$ are disjoint sections of the ruled surfaces. To distinguish $\mathrm{F}_{a}, \mathrm{~F}_{b}$ from their proper transforms, the proper transforms of $\mathrm{F}_{a}$ and $\mathrm{F}_{b}$ are denoted by $\tilde{\mathrm{F}}_{a}$ and $\tilde{\mathrm{F}}_{b}$. Let us first prove (i). $\left(\tilde{\mathrm{F}}_{a} \cap E\right)_{E}^{2}=\left(\left(\tilde{\mathrm{F}}_{a} \cap E\right) \cdot\left(\tilde{\mathrm{F}}_{a} \cap E\right)\right)_{E}=\left(\tilde{\mathrm{F}}_{a} \cdot \tilde{\mathrm{F}}_{a} \cdot E\right)_{Y}=\left(\tilde{\mathrm{F}}_{a}+E-\right.$ $\left.E \cdot \tilde{\mathrm{F}}_{a} \cdot E\right)=\left(p * \mathrm{~F}_{a}-E \cdot \tilde{\mathrm{F}}_{a} \cdot E\right)=\left(p^{*} \mathrm{~F}_{a} \cdot \tilde{\mathrm{F}}_{a} \cdot E\right)_{Y}-\left(E \cdot \tilde{\mathrm{F}}_{a} \cdot E\right)=\left(\mathrm{F}_{a} \cdot p_{*}\left(\tilde{\mathrm{F}}_{a} \cdot E\right)\right)_{X}$ $-(-a)=-b+a$.

Similarly we get $\left(\mathrm{F}_{b} \cap E\right)_{E}^{2}=a-b$. Therefore $E$ is isomorphic to $\mathrm{F}_{a-b}$. Since $a-b>0$, the arrow on $E \simeq \mathrm{F}_{a-b}$ points from $\mathrm{F}_{a}$ to $\mathrm{F}_{b}$. The second assertion is proved by the same method.

Remark (8.5.5). In the diagram it is convenient to extend the arrow to the ruled surface $\mathbf{F}_{0}=\mathbf{P}^{1} \times \mathbf{P}^{1}$. To be precise, for example by definition the diagram

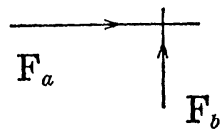

$$
(a, b \geqslant 1)
$$

signifies that the divisors intersect transversely, $\left(\mathrm{F}_{a} \cap \mathrm{F}_{b}\right)_{\mathrm{F}_{a}}^{2}=-a,\left(\mathrm{~F}_{a} \cap \mathrm{F}_{b}\right)_{\mathrm{F}_{a}}^{2}$ $=-b$ and $\mathrm{F}_{a} \cap \mathrm{F}_{b}$ is a section of the ruled surfaces $\mathrm{F}_{a}, \mathrm{~F}_{b}$. Let us allow here $a=0$ or $b=0$ and then an arrow means one intersection number is greater than or equal to the other. The diagram

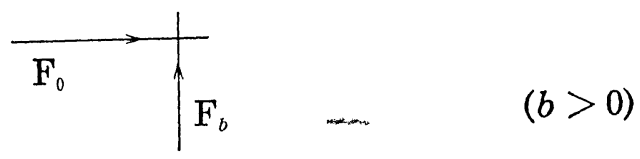

shows that $\mathrm{F}_{0} \cap \mathrm{F}_{b}$ is a section of the ruled surfaces $\mathrm{F}_{0}, \mathrm{~F}_{b}$ and $\left(\mathrm{F}_{0} \cap \mathrm{F}_{b}\right)_{\mathrm{F}_{b}}^{2}$ $=0$ and $\left(\mathrm{F}_{0} \cap \mathrm{F}_{b}\right)_{\mathrm{F}_{0}}^{2}=-b$.

Therefore the diagram (8.5.5) is equivalent to

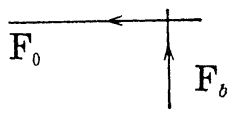

This remark being done, Lemma (8.5.4) (i) holds for $a \geq b \geq 0$ and (ii) is correct for $a, b \geq 0$. 
It follows from Lemma (8.3),

(8.6) the set of the $G$-invariant divisors on $\mathrm{F}_{m, n}^{\ell}$ are described by,

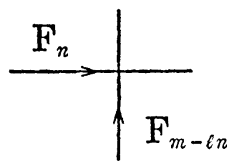

$$
\text { if } m-\ell n>0
$$

or by,

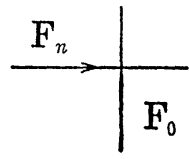

$$
\text { if } m-\ell n=0 \text {. }
$$

Let us treat only the case $m-\ell n>0$ because the case $m-\ell n=0$ is treated without any essential modification. There is only one choice of the center to blow up $\mathrm{F}_{m, n}^{\ell}$ equivariantly: the intersection $\mathrm{F}_{n} \cap \mathrm{F}_{m-\ell n}$. Let us blow-up $\mathrm{F}_{m, n}^{\ell}$ at $\mathrm{F}_{n} \cap \mathrm{F}_{m-\ell n}: \pi_{1}: X_{1} \rightarrow \mathrm{F}_{m, n}^{\ell}$, we get a new diagram of $G$-invariant divisors

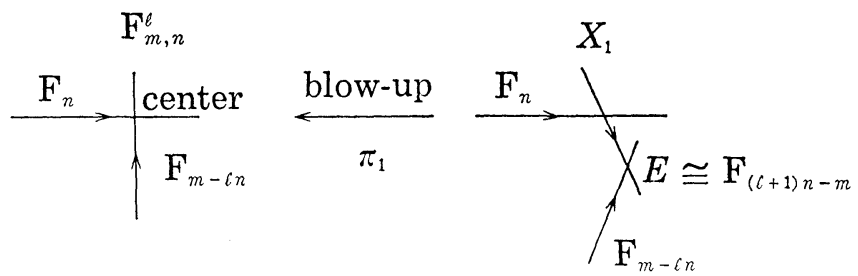

by Lemma (8.5.4).

We can now show that we can blow down $\mathrm{F}_{m-\ell n}$ in $X_{1}$ to $\mathbf{P}^{1}$ and we get $X_{m, n}^{\ell+1}$. This procedure is called the elementary transformation applied for the $\mathbf{P}^{1}$-bundle $f_{m, n}^{\ell}: \mathrm{F}_{m, n}^{k} \rightarrow \mathrm{F}_{n}$ with center $D_{1}^{\ell} \cap D_{2}^{\ell}$ (see Maruyama [Mar 2]). Similarly the $\mathbf{P}^{1}$-bundle $f_{m, n}^{k}: \mathrm{F}_{m, n}^{k} \rightarrow \mathrm{F}_{n}$ and the $\mathbf{P}^{1}$-bundle $f_{m, n}^{k+1}: \mathrm{F}_{m, n}^{k+1}$ $\rightarrow \mathrm{F}_{n}$ are related one another by equivariant elementary transformations. Since $\mathrm{F}_{m, n}^{\ell}$ is a $\mathbf{P}^{1}$-bundle over $\mathrm{F}_{n}$ obtained from the line bundle $\operatorname{Spec}\left(S\left(\mathcal{O}_{\mathrm{F}_{n}}(-m)\right) \oplus \mathcal{O}_{\mathrm{F}_{n}}\left(-\ell C_{\infty}\right)\right)$ by adding the $\infty$-section, we have $\mathcal{O}_{D_{2}^{\ell}}\left(D_{2}^{\ell}\right) \cong$ $\mathcal{O}_{\mathrm{F}_{n}}(-m) \otimes \mathcal{O}_{\mathrm{F}_{n}}\left(-\ell C_{\infty}\right)$ hence, denoting by $f$ the fibre of the ruled surface $D_{2}^{\ell} \cong \mathrm{F}_{n},\left(f \cdot D_{2}^{\ell}\right)_{\mathrm{F}_{m, n}^{\ell}}=-\ell$.

(8.8) If $\ell=1$, we can equivariantly blow down $D_{2}^{\ell} \cong \mathrm{F}_{n}$ to get $\mathrm{F}_{m n}^{1} \rightarrow$ $\mathbf{P}\left(\mathcal{O}_{\mathbf{P} 1} \oplus \mathcal{O}_{\mathbf{P} 1}(-m) \oplus \mathcal{O}_{\mathbf{P} 1}(-n)\right)$.

(8.9) Let us blow up $X_{1}$ at $\mathrm{F}_{(\ell+1) n-m} \cap \mathrm{F}_{n}: \pi_{2}: X_{2} \rightarrow X_{1}$, where $\mathrm{F}_{(\ell+1) n-m}$ 
is the exceptional divisor for $\pi_{1}$ and $F_{n}$ is the proper transform $\pi_{1}^{-1}\left[D_{2}\right]$. It follows from Lemma (8.5.4)
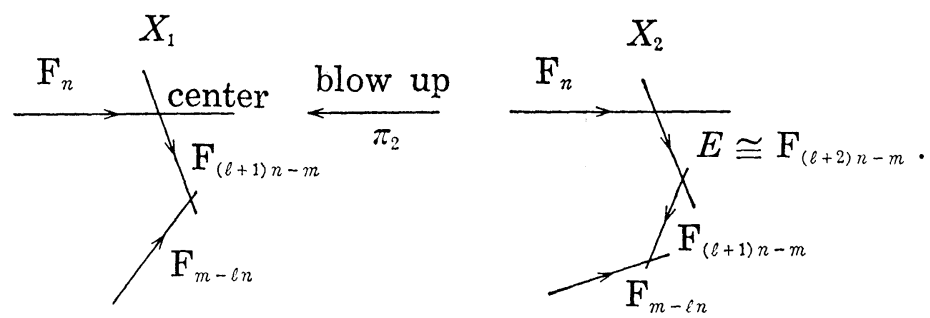

If we continue blowing up $j$-times on the invariant curve $\left(\cong \mathbf{P}^{1}\right)$ on $\mathrm{F}_{n}$, we get

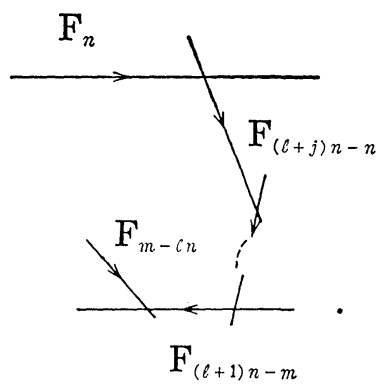

(8.10) Let us blow up $X_{1}$ at $\mathrm{F}_{(\ell+1) n-m} \cap \mathrm{F}_{m-\ell n}: \pi_{3}: X_{3} \rightarrow X_{1}$. We get by Lemma (8.5.4) a diagram
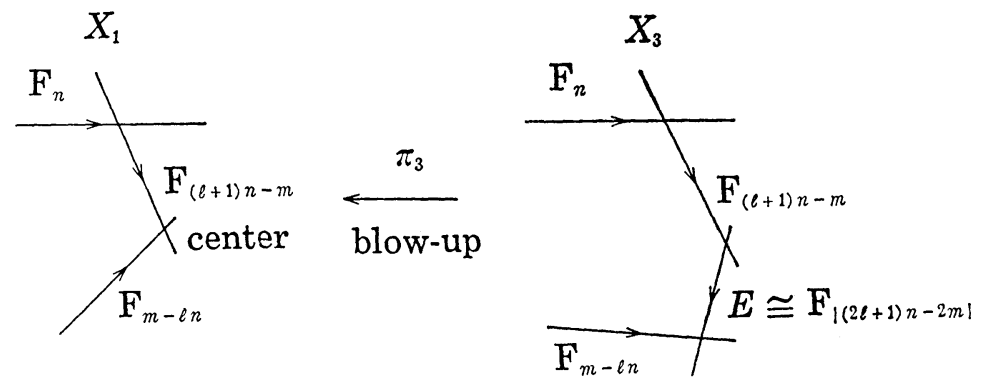

if $(2 \ell+1) n-2 m>0$, otherwise the arrow on $E$ should be reversed. Assume now that $(2 \ell+1) n-2 m=0$ so that $E \cong \mathbf{P}^{1} \times \mathbf{P}^{1}=\mathrm{F}_{0}$ and we can choose a $G$-invariant section $L$ of $\mathbf{P}^{1} \times \mathbf{P}^{1}$ disjoint from $F_{(\ell+1) n-m} \cap E$ and $F_{m-\ell n} \cap E$.

(8.11) Let us blow up $X_{3}$ at $L: \pi_{4}: X_{4} \rightarrow X_{3}$. Denoting by $E$ the exceptional divisor for $\pi_{4}$, we can calculate the intersection number $\left(E . \mathbf{P}^{1} \times\right.$ $\left.\mathbf{P}^{1} . \mathbf{P}^{1} \times \mathbf{P}^{1}\right)=\ell n-m$. In fact let 
(8.11.1)

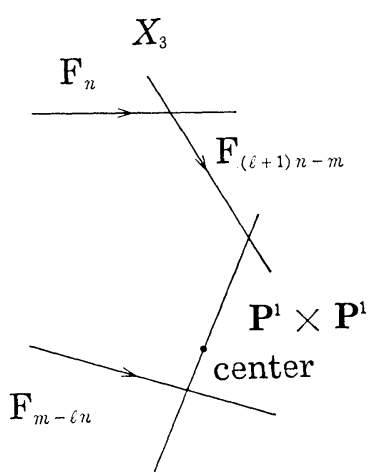

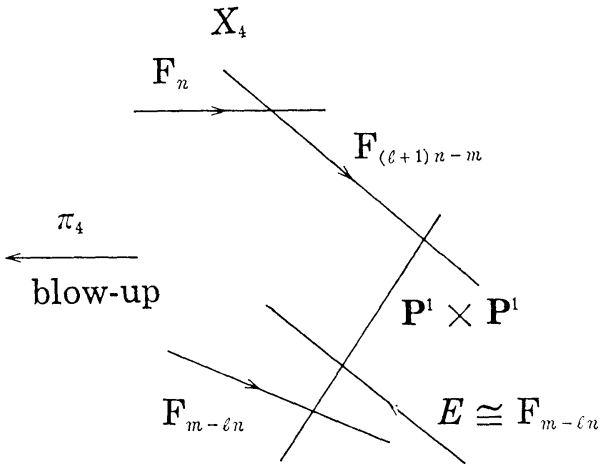

be the diagrams for $X_{3}$ and $X_{4}$. Then we have $\left(E . F_{0} . F_{0}\right)_{X_{4}}=\left(E . F_{0} . F_{0}+E\right.$ $-E)_{X_{4}}=\left(E . F_{0} \cdot\left(\pi_{0}^{*} F_{0}\right)-E\right)_{X_{4}}=\left(E . F_{0} \cdot \pi_{4}^{*} F_{0}\right)_{X_{4}}-\left(E . F_{0} . E\right)_{X_{4}}=\left(E . F_{0} \cdot \pi_{4}^{*} F_{0}\right)_{X_{4}}$ $=\left(\pi_{4 *} E \cap F_{0} . F_{0}\right)_{X_{3}}=\left(F_{m-\ell n} \cap F_{0} . F_{0}\right)=\ell n-m$. Since by Lemma (1.11) $\mathrm{SL}_{2}$ operates non-trivially on $E$ leaving invariant the intersection $E \cap \mathbf{P}^{1} \times \mathbf{P}^{1}$, then it follows from (1.3.3) $E-\mathrm{F}_{m-\ell n}$ and $E \cap \mathbf{P}^{1} \times \mathbf{P}^{1}$ is the $\infty$-section of $E$.

(8.12) Let us blow up $X_{4}$ at another section of $\mathrm{F}_{n}$ disjoint from $E \cap \mathbf{P}^{1} \times \mathbf{P}^{1} ; \pi_{5}: X_{5} \rightarrow X_{4}$. As $\mathrm{SL}_{2}$ operates on $E$ non-trivially leaving the intersection $\mathrm{F}_{n} \cap E$ by Lemma (1.11), for the same reason as in (8.11) we get

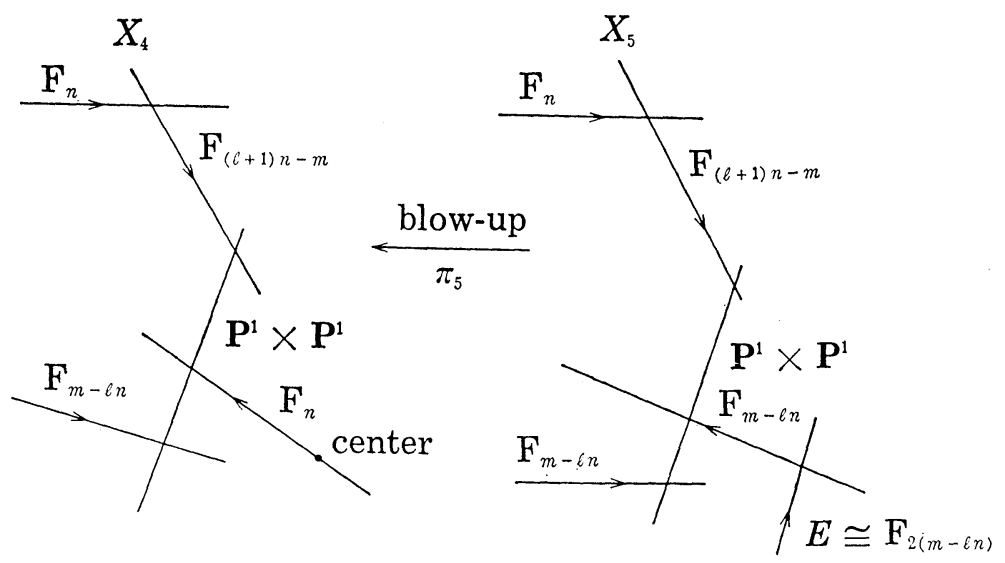

Lemma (8.12.2). Let $X$ be a (successive) equivariant blow-up of $\mathrm{F}_{m, n}^{\ell}$. Then a G-invariant irreducible divisor on $X$ is isomorphic to $\mathrm{F}_{a}, a \geq 0$ with $a \in \mathbf{Z} m+\mathbf{Z n}$. There is at most only one G-invariant divisor on $X$ isomorphic to $\mathrm{F}_{0}$ (see also Lemma (8.14)).

Proof. This is a cosequence of Lemma (8.5.4), Remark (8.5.5) and 
the argument of (8.11.1) and (8.12.1).

Lemma (8.13). Let $(G, X) \in C\left(\right.$ Aut $\left.^{0} \mathrm{~F}_{m, n}^{\prime}, \mathrm{F}_{m, n}^{\prime}\right)=\mathscr{C}(\mathrm{J} 9 ; m, n)$. We assume $(m, n) \geq 2$. Then (a) there is no G-invariant divisor on $X$ which is isomorphic either to $\mathbf{P}^{2}$ or $\mathrm{F}_{1}$. (b) There is no G-fixed point on $X$.

Proof. We have an equivariant birational map $\varphi: X \cdots \rightarrow \mathrm{F}_{m, n}^{\ell}$. We can eliminate the indeterminacy of $\varphi$ by equivariantly blowing up $X$;

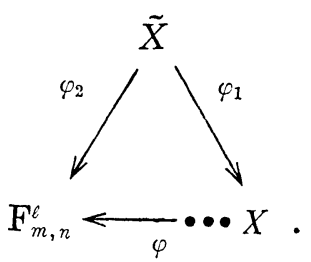

Since there is no fixed point on $\mathrm{F}_{m, n}^{\ell}$ by Lemma (8.3), $\varphi_{2}$ is also an equivariant blow-up by Theorem (1.7). If there were a $G$-invariant divisor isomorphic to $\mathrm{P}^{2}$ or $\mathrm{F}_{1}$ on $X$, then it proper transform would be a $G$ invariant divisor on $X$ isomorphic to $\mathbf{P}^{2}$ or to $\mathrm{F}_{1}$. This contradicts Lemma (8.12.2) since $(m, n) \geq 2$. The assertion (a) is proved. If we blow up $X$ at a $G$-fixed point, (b) follows from (a).

Lemma (8.14). Let $(G, \tilde{X}) \in \mathscr{C}\left(\right.$ Aut $\left.^{0} \mathrm{~F}_{m, n}^{\prime}, \mathrm{F}_{m, n}^{\prime}\right)=\mathscr{C}(\mathrm{J} 9 ; m, n)$ and $\left(\mathrm{Id}, \varphi_{2}\right)$ : $(G, \tilde{X}) \rightarrow\left(G, \mathrm{~F}_{m, n}^{e}\right)$ be an equivariant blow-up, where we choose the integer $\ell$ as before: $m=n \ell+r, 0 \leqq r<n$. We denote by $S$ the set of all the irreducible reduced effective G-invariant divisors on $\tilde{X}$. Then (a) $S$ is a finite set. (b) The subvariety $\cup_{D \in S} D \subset \tilde{X}$ is connected. (c) $S$ satisfies the condition (1), (2), (3) of Definition (8.4). (d) If a divisor $D \in S$ is exceptional for $\varphi_{2}$ and if there is only one divisor $D^{\prime} \in S, D \neq D^{\prime}$ ) with $D \cap D^{\prime} \neq \varnothing$, then $\left(D \cap D^{\prime}\right)_{D}^{2}>0$. (e) For any G-invariant irreducible reduced curve $C$, there exists a G-invariant irreducible reduced effective divisor $D$ such that $C$ lies on $D$ such that $C$ is a section of the rational ruled surface $D \cong \mathrm{F}_{t}$ and $\left(C^{2}\right)_{D}= \pm t$.

Remark (8.14.1). Later we shall not use (d). But we need (a), (b), (c), (d) to prove (a), (b), (c) inductively.

Proof. First of all, we notice that the center of each step of $\varphi_{2}$ is a $G$-orbit by Lemma (8.13). Therefore the morphism $\varphi_{2}: \tilde{X} \rightarrow \mathrm{F}_{m, n}^{l}$ maps the centers onto the unique $G$-invariant curve $D_{1}^{\ell} \cap D_{2}^{\ell}$ on $\mathrm{F}_{m, n}^{\ell}$. This proves 
(b) by induction. Now let $\mathrm{SL}_{2} \rightarrow G$ be a non-trivial morphism and hence give a semi-simple part of $G$ by Corollary (4.17), [U4]. Then by Lemma (1.11) for any $D \in S, \mathrm{SL}_{2}$ operates non-trivially on $D$. We prove the Lemma by induction on the length of the sequence $\varphi_{2}$ of blow-ups at smooth irreducible centers or on the Picard number $\rho(\tilde{X})$ of $\tilde{X}$. The Lemma holds for $\mathrm{F}_{m, n}^{e}$ by Lemma (8.3). The assertion (a) is evidently proved by induction. The remaining assertions (c), (d), (e) are not obvious. Let us factor $\varphi_{2}=\varphi_{3} \circ \varphi_{4}$ :

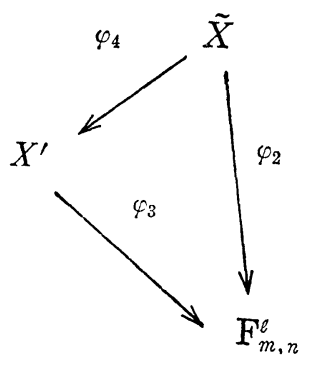

where $\varphi_{3}$ is a sequence of $G$-equivariant blow-ups for which the Lemma holds and $\varphi_{4}$ is a $G$-equivariant blow-up at an irreducible $G$-invariant center, which is a curve $C$ isomorphic to $\mathbf{P}^{1}$ by Lemma (8.3). We assume that the Lemma holds for $X^{\prime}$ and prove it for $X$. There are three possibilities:

(i) there exists 2 divisors $D_{1}, D_{2} \in S^{\prime}$ such that $C=D_{1} \cap D_{2}$, where $S^{\prime}$ denotes the set of $G$-invariant irreducible reduced effective divisors on $X^{\prime}$ (cf. Fig. (8.14.2. (i)), Examples (8.7), (8.9.1), (8.9.2) and (8.10)).

(ii) $C$ lies on the unique $G$-invariant divisor $D$ on $X^{\prime}$.

We may assume in the case of (ii) that $D$ is exceptional for $\varphi_{3}$ by Lemma (8.3). For otherwise $C$ is in the case (i) by Lemma (8.3).

The case (ii) is divided into 2 subcases:

(ii a) $C$ lies on the unique $G$-invariant divisor $D$ on $X^{\prime}$ and $D$ is isomorphic to $\mathbf{P}^{1} \times \mathbf{P}^{1}$ and exceptional for $\varphi_{3}$ (cf. Fig. (8.14.2. (ii a)).

Fig (8.14.2)

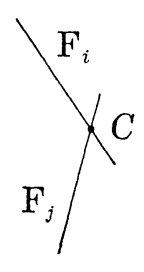

(i)

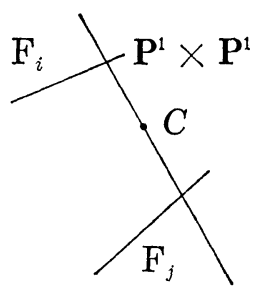

(ii a)

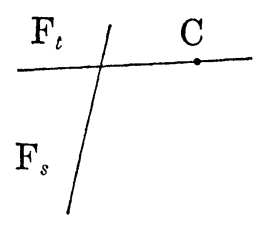

(ii b) 
Example (8.11)),

(ii b) $C$ lies on the unique $G$-invariant divisor $D$ on $X^{\prime}$ and $D$ is isomorphic to $F_{t}(t>0)$ and exceptional for $\varphi_{3}$ (cf. Fig. (8.14.2. (ii b)), Example (8.12)).

In the case (i), it is evident that Lemma (8.14) holds for $\tilde{X}$ by (1.3.2) and Lemma (1.11):
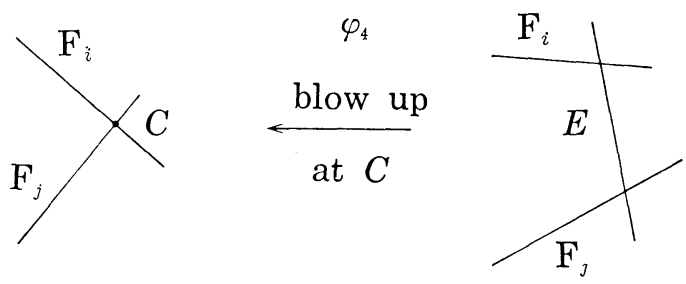

Let us study the case (ii a). In this case, it follows from (e) for $C$ on $X^{\prime}$ that $(C, C)_{D}=0$. By the assertions (c), (d), (e) for $X^{\prime}$, we have:
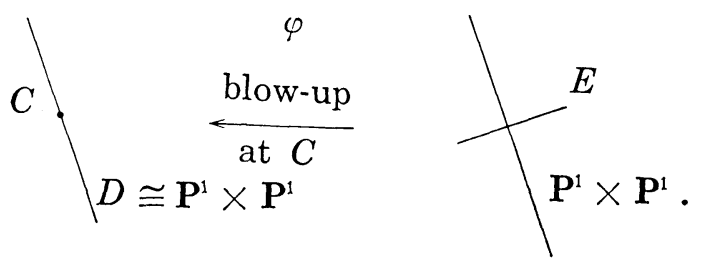

Namely the exceptional divisor $E$ for $\varphi_{4}$ intersects on $\tilde{X}$ with the unique $G$-invariant divisor which is the proper transform of $D$. Let us calculate the intersection number: by the projection formula $-n=\left(C_{\infty} . C_{\infty}\right)_{\mathrm{F}_{n}}$ $=\left(f_{m, n}^{\ell} C_{\infty} .\left(D_{1}^{\ell} \cap D_{2}^{\ell}\right)\right)_{\mathrm{F}_{m}^{\prime},}=\left(\left(f_{m, n}^{\ell} \circ \varphi_{2}\right) * C_{\infty} . E \cdot \varphi_{4}^{-1}[D]\right)_{\tilde{X}}=\left(\left\{a E+a \varphi_{4}^{-1}[D]+(\mathrm{a}\right.\right.$ divisor disjoint from $\left.\left.\left.E \cap \varphi_{4}^{-1}[D]\right)\right\} . E \cdot \varphi_{4}^{-1}[D]\right)_{\tilde{X}}$ with an integer $a>0$, $=a\left(E . E \cdot \varphi_{4}^{-1}[D]\right)_{\tilde{X}}+a\left(\varphi_{4}^{-1}[D] . E \cdot \varphi_{4}^{-1}[D]\right)_{\tilde{X}}=a\left(\varphi_{4}^{-1}[D] . E \cdot \varphi_{4}^{-1}[D]\right)_{\tilde{X}}$ by the assumption (e). This proves that for the new born $G$-invariant divisor $E$ the assertions (c), (d), (e) hold by (1.3.3) since $\mathrm{SL}_{2}$-operates non-trivially on any $G$-invariant divisor on $\tilde{X}$ by Lemma (1.11). The Lemma is proved for $\tilde{X}$ in this case. Now we study the last case (ii b). By (1.3.3), Lemma (1.11) and by the assertion (c), (d), (e) for $X^{\prime}$ we have:
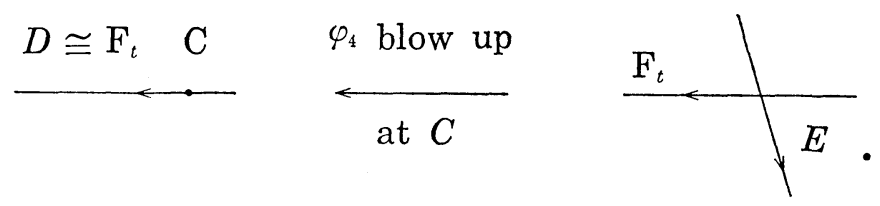
Namely the exceptional divisor $E$ for $\varphi_{4}$ intersects with the unique $G$-invariant divisor which is the proper transform of $D$. We calculate the intersection number as above: $-n=\left(C_{\infty} . C_{\infty}\right)_{\mathrm{F}_{n}}=\left(\left(f_{m, n}^{\ell} \circ \varphi_{2}\right) * C_{\infty} \cdot E \cdot \varphi_{4}^{-1}[D]\right)_{\tilde{x}}$ $=\left(\left\{a E+a \varphi_{4}^{-1}[D]+\left(\text { a divisor disjoint from } E \cap \varphi_{4}^{-1}[D]\right\} \cdot E \cdot \varphi_{4}^{-1}[D]\right)_{\tilde{X}}\right.$, with an integer $a>0,=a\left(E \text {. E. } \varphi_{4}^{-1}[D]\right)_{\tilde{X}}+a\left(\varphi_{s}^{-1}[D] \text {. E. } \varphi_{4}^{-1}[D]\right)_{\tilde{X}}$. Hence $\left(E . E \cdot \varphi_{4}^{-1}[D]\right)_{\tilde{X}}$ $<0$ since $\left(\varphi_{4}^{-1}[D] \text {. E. } \varphi_{4}^{-1}[D]\right)_{\tilde{x}}=(C, C)_{D}>0$. This shows that the new born divisor $E$ satisfies the assertions (c), (d), (e) of the Lemma for the same reason as in the case (ii a). This proves the Lemma for $\tilde{X}$ in the case (ii b) and hence the Lemma.

Corollary (8.15). We assume $(m, n) \geq 2$. Let $(G, X) \in \mathscr{C}\left(\right.$ Aut $\left.^{0} \mathrm{~F}_{m, n}^{\prime} \mathrm{F}_{m, n}^{\prime}\right)$ $=\mathscr{C}(\mathrm{J} 9 ; m n),(G, \tilde{X}) \in \mathscr{C}(\mathrm{J} 9 ; m, n),\left(\operatorname{Id}, \varphi_{1}\right):(G, \tilde{X}) \rightarrow\left(G, \mathrm{~F}_{m, n}^{e}\right)$ and $\left(\operatorname{Id}, \varphi_{1}\right)$ : $(G, \tilde{X}) \rightarrow(G, X)$ equivariant blow-ups with smooth centers. Then there is no G-invariant divisor $D$ isomorphic to $\mathbf{P}^{1} \times \mathbf{P}^{1}$ blown-down to $\mathbf{P}^{1}$ along different projections by $\varphi_{1}$ and $\varphi_{2}$ : the restriction of $\varphi_{i}$ on $D \cong \mathbf{P}^{1} \times \mathbf{P}^{1}$ coincides with the projection $p_{i}: \mathbf{P}^{1} \times \mathbf{P}^{1} \rightarrow \mathbf{P}^{1}$ onto the $i$-th factor $(i=1,2)$.

Proof. Assume that the existence of such a divisor $D$. It follows from Lemma (8.14) that $G$ leaves invariant $a \times \mathbf{P}^{1} \subset \mathbf{P}^{1} \times \mathbf{P}^{1}$ for a suitable point $a \in \mathbf{P}^{1}$. Therefore $\varphi_{1}\left(a \times \mathbf{P}^{1}\right)$ is a $G$-fixed point on $X$, which contradicts Lemma (8.13).

Remark (8.15.1). We can prove Corollary (8.15) directly. It follows from Lemma (8.12.2) that in any (successive) blow-up of $\mathrm{F}_{m, n}^{\ell}$, there is at most 1 invariant divisor $E$ is isomorphic to $\mathbf{P}^{1} \times \mathbf{P}^{1}$. Let $\ell$ be the line $\mathbf{P}^{1}$ to be contracted to a new direction. Then there is an invariant divisor $D$ such that $\ell$ is numerically equivariant to $E \cap D$. The divisor $D$ is isomorphic to $\mathrm{F}_{a}$ for a suitable $a \in \mathbf{Z} m+\mathbf{Z} n$. Since $E$ is contracted, $-1=(E \cdot \ell)=($ E.E. $D)= \pm a$. This is a contradiction as we assume $(m, n) \geq 2$.

Lemma (8.16). Under the hypothesis $(m, n) \geq 2$, none of the cases (3.3.2), (3.3.3), (3.3.4) and (3.3.5) of Theorem (3.3), [Mo] occurs.

Proof. The cases (3.3.2), (3.3.5) are avoided by (a), Lemma (8.13). The case (3.3.4) is excluded by (b), Lemma (8.13) since the singular point of the $G$-invariant divisor is a $G$-fixed point.

Assume now that (3.3.3) is the case. Let $\mathrm{F}_{m, n}^{e} \ll \cdots X_{1}$ be an equivariant birational map, where we choose $\ell$ as before. Since there appears no fixed point by Lemma (8.13), there exist equivariant blow-ups $\varphi_{2}: \tilde{X} \rightarrow \mathrm{F}_{m, n}^{e}$ 
and $\varphi_{1}: \tilde{X} \rightarrow X$ such that $\varphi_{2} \circ \varphi_{1}^{-1}=\varphi$ by Theorem (1.7). The proper transform $\varphi_{1}^{-1}[D]$ of the divisor $D \cong \mathbf{P}^{1} \times \mathbf{P}^{1}$ of case (3.3.3) in [Mo] is $G$-invariant $\mathbf{P}^{1} \times \mathbf{P}^{1}$ on $\tilde{X}$. Therefore $\varphi_{2}\left(\mathbf{P}^{1} \times \mathbf{P}^{1}\right)$ is a $G$-invariant divisor and hence by Lemma (8.3) contained in $D_{1}^{\ell}$ : in fact $\varphi_{2}\left(\mathbf{P}^{1} \times \mathbf{P}^{1}\right)=D_{1}^{\ell} \cap D_{2}^{\ell}$ unless $m=\ell n$. Since $\mathrm{SL}_{2}$ operates on $\mathrm{F}_{m, n}^{\prime}$, it operates also on $D \cong \mathbf{P}^{1} \times \mathbf{P}^{1}$. There are 3 possibilities: (1) $\mathrm{SL}_{2}$ fixes all the point of $\mathbf{P}^{1} \times \mathbf{P}^{1}$, (2) $\mathrm{SL}_{2}$ operates through one of the factors of $\mathbf{P}^{1} \times \mathbf{P}^{1}$, (3) $\mathrm{SL}_{2}$ operates diagonally on $\mathbf{P}^{1} \times \mathbf{P}^{1}$. The case (1) never occurs by Lemma (1.11). We now exclude the case where $\mathrm{SL}_{2}$ operates on $D$ through one of the factors of $\mathbf{P}^{1} \times \mathbf{P}^{1}$, say the second. It follows from the assumption (cf. Theorem (3.3) [Mo]) that $s \times \mathbf{P}^{1}$ and $\mathbf{P}^{1} \times t$ are numerically equivalent. Taking $s$ general, we may assume that $s \times \mathbf{P}^{1}$ is disjoint from the center of the blow-up $\varphi_{1}$ since $\varphi_{1}$ is equivariant. Then $\varphi_{1}^{*} s \times \mathbf{P}^{1}$ is numerically equivalent to $s \times \mathbf{P}^{1}$ in the proper transform $\varphi^{-1}\left[\mathbf{P}^{1} \times \mathbf{P}^{1}\right]$ and hence $\varphi_{2 *} \varphi_{1}^{*} s \times \mathbf{P}^{1}$ is numerically equivalent to either $D_{1}^{\ell} \cap D_{2}^{\ell}$ or to a 0 -section $C_{0}$ of the rational ruled surface $D_{1}^{\ell}$ since $\varphi_{2}$ is $\mathrm{SL}_{2}$-equivariant and $\varphi_{2 *} \varphi_{1}^{*} s \times \mathbf{P}^{1}$ is $\mathrm{SL}_{2}$-invariant curve on $D_{1}^{\ell}$. It follows from Corollary (8.15) that $\varphi_{2 *} \varphi_{1}^{*}\left(\mathbf{P}^{1} \times t\right)$ is numerically equivalent to $a C_{1}+b C_{2}$ for some integers $a, b \geqq 0$, where $C_{1}$ (resp. $C_{2}$ ) is a fibre of the ruled surface $D_{1}^{\ell}$ (resp. $D_{2}^{\ell}$ ). $\pi: \mathrm{F}_{m, n}^{\ell} \rightarrow \mathrm{F}_{n} \rightarrow \mathbf{P}^{1}$ denote the fibration which is respected by $G$ and we put $\pi^{*} \mathcal{O}_{\mathbf{p}_{1}}(1)=H$ (see Proposition (4.13), [U4]). Then $\left(H . C_{0}\right)=\left(H . D_{1} \cap D_{2}\right)=1$ but $\left(H . C_{1}\right)=\left(H . C_{2}\right)$ $=0$. Therefore $\left(\varphi_{1}^{*}\left(s \times \mathbf{P}^{1}\right) \cdot \varphi_{2}^{*} H\right)=\left(\varphi_{2 *} \varphi_{1}^{*}\left(s \times \mathbf{P}^{1}\right) . H\right)$ which is equal to $\left(C_{0} . H\right)$ or $\left(\left(D_{1}^{\ell} \cap D_{2}^{\ell}\right) . H\right)$ hence to 1 and $\left(\varphi_{1}^{*}\left(\mathbf{P}^{1} \times t\right) . \varphi_{2 *} H\right)=\left(\varphi_{2 *} \varphi_{1}^{*}\left(\mathbf{P}^{1} \times t\right) . H\right)$ $\left(\left(a C_{1}+b C_{2}\right) . H\right)=0$. This contradicts the numerical equivalence of $\mathbf{P}^{1} \times t$ and $s \times \mathbf{P}^{1}$ on $X$. Now we have to exclude the last possibility; the diagonal operation of $\mathrm{SL}_{2}$ on $D \cong \mathbf{P}^{1} \times \mathbf{P}^{1}$. Assume to the contrary. Then $s \times \mathbf{P}^{1}-\mathbf{P}^{1} \times t \sim 0$ and $0=\varphi_{1}^{*}\left(s \times \mathbf{P}^{1}-\mathbf{P}^{1} \times t\right)=\left(s \times \mathbf{P}^{1}\right.$ in $\left.\varphi_{1}^{-1}[D]\right)$ - $\left(\mathbf{P}^{1} \times t\right.$ in $\left.\varphi_{1}^{-1}[D]\right)$ hence taking $\varphi_{2 *}, \varphi_{2 *}\left(s \times \mathbf{P}^{1}\right.$ in $\left.\varphi_{1}^{-1}[D]\right)$ is numerically equivalent to $\varphi_{2 *}\left(\mathbf{P}^{1} \times t\right.$ in $\left.\varphi_{1}^{-1}[D]\right)$ for any $s, t \in \mathbf{P}^{1}$. Let us put $C_{1}=\varphi_{2}(s \times$ $\mathbf{P}^{1}$ in $\left.\varphi_{1}^{-1}[D]\right)$ and $C_{2}=\varphi_{2}\left(\mathbf{P}^{2} \times t\right.$ in $\left.\varphi_{1}^{-1}[D]\right)$. We have one of the following: (1) $\varphi_{2}$ blows down $\varphi_{1}^{-1}[D]=\mathbf{P}^{1} \times \mathbf{P}^{1} \rightarrow \mathbf{P}^{1}$. (2) $\varphi_{2}$ is biregular at $\mathbf{P}^{1} \times \mathbf{P}^{1}$. In the first case one of $C_{i}(i=1,2)$ is reduced to a point and the other coincides with the unique $G$-invariant curve $D_{1}^{\ell} \cap D_{2}^{\ell}$. Thus $D_{1}^{\ell} \cap D_{2}^{\ell}$ is numerically equivalent to 0 . This is absurd and the case (1) never happens. In the second case, $m=\ell n$ and $D_{1} \cong \mathbf{P}^{1} \times \mathbf{P}^{1}, s \times \mathbf{P}^{1}$ and $t \times \mathbf{P}^{1} \subset D_{1}$ $=\mathbf{P}^{1} \times \mathbf{P}^{1}$ are numerically equivalent, which does not happen (compare the intersection numbers $\left.\left(D_{1} . C_{i}\right), i=1,2\right)$. 
Lemma (8.17). Let $(G, X) \in \mathscr{C}(\mathrm{J} 9 ; m, n)$. Let $\phi: X \rightarrow Y$ be the morphism of (3.5.1), Theorem (3.5), [Mo]. Then the surface $Y$ is isomorphic to $\mathrm{F}_{n}$ and $X$ is a $\mathbf{P}^{1}$-bundle over $\mathrm{F}_{n}$.

Proof. By Castelnuovo's Theorem [Z] $Y$ is rational and $G$ has an open orbit on $Y . \phi$ induces a morphism $\psi: G \rightarrow \operatorname{Aut}^{0} Y$. A semi-simple part of $G$ is $\mathrm{SL}_{2}$ and its image under $\psi$ does not reduce to 1 . For otherwise $\mathrm{Ker} \psi$ would be isogeneous to $\mathrm{SL}_{2}$ since the general fibre of $\phi$ is isomorphic to $\mathbf{P}^{1}$. Hence the semi-simple part of $G$ would be normal, which contradicts Corollary (4.17), [U4]. Therefore $\mathrm{SL}_{2}$ operates nontrivially on $Y$. Since the unipotent radical of $G$ is not abelian by Corollary (4.17) [U4], it follows from Lemma (4.4), [U4] and Lemma (1.8) that the unipotent radical of $G$ operates non-trivially on $Y, Y$ is a ruled surface and $G$ operates on $Y$ with orbit decomposition: (open orbit) $\cup \mathbf{P}^{1}$. By Lemma (1.13), $X$ is a $\mathbf{P}^{1}$-bundle over $Y$. We show that $Y$ is isomorphic to $\mathrm{F}_{n}$. First assume that $Y \cong \mathrm{F}_{n}$. We denote by $W_{j}$ the irreducible $\mathrm{SL}_{2}$-module of rank $j+1$. It follows from Corollary (4.17), [U4] that the irreducible $\mathrm{SL}_{2}$-module $W_{m-n}(m-n \geqq 1)$ operates on $\mathrm{F}_{m, n}^{\prime} ; x \mapsto x, y \mapsto y$, $z \mapsto z+y \varphi_{m-n}(x)$, where $\varphi_{m-n}(x) \in k[x]$ with $\operatorname{deg} \varphi_{m-n}(x) \leqq m-n$. Hence the map $\mathrm{F}_{m, n}^{k} \rightarrow \mathrm{F}_{n}$ is generically the quotient by $W_{m-n}$. Since $W_{m-n}$ operates trivially on $\mathrm{F}_{m}$, the $\mathbf{P}^{1}$-bundle is generically the quotient of $X$ by $W_{m-n}$ and hence $Y$ is $G$-equivariantly isomorphic to $\mathrm{F}_{n}^{\prime}$. Consequently $Y$ is Aut ${ }^{0} \mathrm{~F}_{n}$-equivariantly isomorphic to $\mathrm{F}_{n}$ by Corollary (4.17), [U4]. Hence $\mathrm{F}_{n} \cong Y$, which contradicts the assumption. Let us now assume that $Y$ is not isomorphic to $\mathrm{F}_{m}$. The using $W_{m} ; x \mapsto x, y \mapsto y, z \mapsto z+\varphi_{m}(x)$ in place of $W_{m-n}$, by the same argument we conclude $\mathrm{F}_{n} \cong Y$.

Lemma (8.18). Let $(G, X) \in \mathscr{C}(\mathrm{J} 9 ; m, n)$. The case (3.5.2) of Theorem (3.5) in [Mo] occurs if and only if $2 n>m>n$ and then $X$ is isomorphic to $\mathbf{P}\left(\mathcal{O}_{\mathbf{p}_{1}}(-m) \oplus \mathcal{O}_{\mathbf{p}_{1}}(-n) \oplus \mathcal{O}_{\mathbf{P}_{1}}\right)$.

Proof. Assume that the case (3.5.2), [Mo] happens and let $\phi: X \rightarrow Y$ be the resulted morphism. $G$ operates on $Y$ and $\phi$ is equivariant. By Lüroth's theorem, $Y$ is isomorphic to $\mathbf{P}^{1}$. Hence induces a morphism $\psi: G \rightarrow \mathrm{PGL}_{2}$. A semi-simple part of $G$ which is isogeneous to $\mathrm{SL}_{2}$, is mapped surjectively onto $\mathrm{PGL}_{2}$. For otherwise there would be a subgroup of $\operatorname{Ker} \psi$ isogeneous to the semi-direct product $\left(U_{m+1} \otimes U_{n+1}\right) \mathrm{SL}_{2}$ by Corollary (4.17), [U4], where $U_{m+1}$ and $U_{n+1}$ denote respectively the irreducible $\mathrm{SL}_{2}$-modules of degree $m+1$ and $n+1$, since $\mathrm{PGL}_{2}$ contains no unipotent 
group bigger than $\mathbf{G}_{a}$. Therefore $\left(U_{m+1} \oplus U_{n+1}\right) \mathrm{SL}_{2}$ would operate on the fibres of $\phi$. We taking $y \in Y$ general, it follows that $\left(U_{m+1} \oplus U_{n+1}\right) \mathrm{SL}_{2}$ would operate almost effectively on the fibre $X_{y}$, which contradicts Theorem (2.25), [U3]. Thus $\psi: G \rightarrow \mathrm{PGL}_{2}$ is surjective and gives the semisimple part of $G$. Hence by letting $G / H \cong \mathrm{F}_{m, n}^{\prime}$ and $B$ a Borel subgroup of $G$ containing $H$, both $\varphi: X \rightarrow Y$ and $\pi: \mathrm{F}_{m, n}^{k} \rightarrow \mathbf{P}^{1}$ are birationally equivalent to $G / H \rightarrow G / X$. In particular $\varphi$ and $\pi$ are birationally equivalent. It follows that $Y$ is a homogeneous space under $G$ and all the fibres of $\varphi$ is a non-singular del Pezzo surface $S$. The surface $S$ is an equivariant completion of $\mathbf{A}^{2}$ under the action: $(y, z) \mapsto(y+a, z+f(y)) f(y) \in k[y]$ with $\operatorname{deg} f(y) \leqq \ell$ by Corollary (4.17), [U4], where $\ell$ is an integer with $m=n \ell+r, 0 \leqq r<n$. Therefore by Theorem 24.3 (ii) Manin [Man] $S$ is either $\mathrm{P}^{2}$ or $F_{1}$ and $\ell=1$. But the $F_{1}$-bundles are excluded in the Mori theory, Theorem (3.5), [Mo]. Thus $S$ is $\mathbf{P}^{2}$. Therefore there exists a vector bundle $E \cong \mathcal{O}(a) \oplus \mathcal{O}(b) \oplus \mathcal{O}, a \leqq b \leqq 0$ such that $\mathbf{P}(E) \cong X$. Looking at End $E$, it follows from Corollary (4.17), [U4] that this is possible if and only if $a=-m, b=-n$. The if part follows from Corollary (4.17), [U4].

Lemma (8.19). Let $(G, X) \in \mathscr{C}(\mathrm{J} ; m, n)$, and $(m, n) \geq 2$. (1) If $m \geq 2 n$, then the Picard number $\rho(X) \geqq 3$. (2) If $2 n>m(>n)$, then $\rho(X) \geqq 2$. (3) Moreover $\rho(X)=2$ if and only if $2 n>m(>n)$ and $X$ is isomorphic to $\mathbf{P}\left(\mathcal{O}_{\mathbf{P}_{1}}(-m) \oplus \mathcal{O}_{\mathbf{P}_{1}}(-n) \otimes \mathcal{O}_{\mathbf{P}_{1}}\right)$.

Proof. Let us first show that $\rho(X) \geqq 2$ in general. Assume $\rho(X)=1$. There exists an equivariant birational map $\chi: \mathrm{F}_{m, n}^{k} \cdots \rightarrow X$, where we fix a large integer $k$. Then we can eliminate the indeterminacy of $\chi$ by equivariantly blowing up $\mathrm{F}_{m, n}^{k}$ :

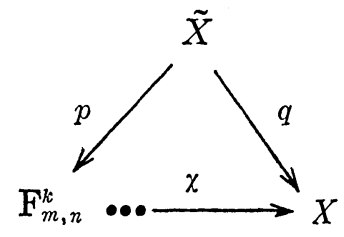

Since there is no fixed point on $X, q$ is also an equivariant blow-up by Throrem (1.7). $\rho\left(\mathrm{F}_{m, n}^{k}\right)=3$ and there are exactly $2 G$-invariant integral divisors $D_{1}^{k}$ and $D_{2}^{k}$ on $\mathrm{F}_{m, n}^{k}$ and they form with $\pi^{*} \mathcal{O}_{\mathbf{P} 1}(1)$ a basis of Pic $\mathrm{F}_{m, n}^{k} \otimes \mathbf{Q}$. Since $\rho(X)=1, q$ should blow-down all the components of $p^{-1}\left(D_{1}^{k} \cup D_{2}^{k}\right)$. 
Sublemma (8.19.1). (a) $q p^{-1}\left(D_{1}^{k} \cup D_{2}^{k}\right)$ is a G-invariant curve $C$. (b) $q^{-1}(y)$ is a 1-cycle on $\tilde{X}$ for any point $y \in C$. (c) Putting $q^{-1}(y)=f$, we have the intersection number $(E . f)=0$ for all but one irreducible components $E$ of $p^{-1}\left(D_{1}^{k} \cup D_{2}^{k}\right)$ and $(E . f)=-1$ for one particular irreducible component $E^{\prime}$.

Proof. We have already observed above (a). (b) follows from Lemma (8.13). Let us write $q$ as a product of the blow-ups $q_{1}: X^{\prime} \rightarrow X$ with irreducible center $C$ and $q^{\prime}: \tilde{X} \stackrel{q^{\prime}}{\longrightarrow} X^{\prime}$. Let us denote $q_{1}^{-1}(C)$ by $E^{\prime}$.

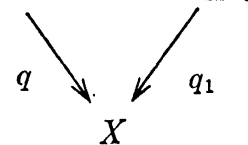

$E^{\prime}$ is the only one $G$-invariant divisor on $X^{\prime}$. For an irreducible component $E$ of $p^{-1}\left(D_{1}^{k} \cup D_{2}^{k}\right)$, we have $(E . f)=\left(E . q^{\prime *}\left(q_{1}^{-1}(y)\right)=\left(q_{*} E \cdot q_{1}^{-1}(y)\right)=0\right.$ or -1 according as $q_{*}^{\prime} E=0$ or $q_{*}^{\prime} E=E^{\prime}$ (notice that $q^{\prime}$ is a blow-down).

q.e.d.

Let us continue the proof of the Lemma. Since as in the proof of Corollary (8.15) $q$ blows-down the exceptional divisors to the same directions as $p,\left(p^{*} D_{2}^{k} . f\right)=\left(D_{2}^{k} \cdot p_{*} f\right)=\left(D_{2}^{k} \cdot f_{1}+f_{2}\right)$, where $f_{i}$ is the fibre of the ruled surface $D_{i}^{k}$. It follows from Sublemma $\left(p^{*} D_{2}^{k} . f\right)=0$ or -1 . But $\left(D_{2}^{k} . f\right)=1,\left(D_{2}^{k} . f\right)=-k$ hence $\left(D_{2}^{k}, f_{1}+f_{2}\right)=1-k$. This is absurd. Now Lemma (8.19) follows from Lemmas (8.16), (8.17) and (8.18).

Theorem (8.20). We assume $(m, n) \geq 2$. Any element of $\mathscr{C}(\mathrm{J} 9 ; m, n)$ $(m>n \geqq 2)$ can be equivariantly blown-down to a relatively minimal element of the ordered set $\mathscr{C}(\mathrm{J} 9 ; m, n)$ : an element $x$ of an ordered set $Z$ is said to be relatively minimal if $x>y$ for $y \in Z$ implies $x=y$. The centers of the blow-down are curves isomorphic to $\mathbf{P}^{1}$.

(1) If $m \geqq 2 n$, the relatively minimal elements in $\mathscr{C}(\mathrm{J} 9 ; m, n)$ are the (Aut $\left.{ }^{0} \mathrm{~F}_{m, n}^{k}, \mathrm{~F}_{m, n}^{k}\right)^{\prime} s(k \geqq \ell)$.

(2) If $2 n>m$, the relatively minimal elements in $\mathscr{C}(\mathrm{J} 9 ; m, n)$ are the $\left(\text { Aut }^{0} \mathrm{~F}_{m, n}^{k}, \mathrm{~F}_{m, n}^{k}\right)^{\prime} s(k>\ell)$ and $\left(\operatorname{Aut}^{0} \mathbf{P}\left(\mathcal{O}_{\mathbf{P}_{1}}(-m) \oplus \mathcal{O}_{\mathbf{P}_{1}}(-n) \oplus \mathcal{O}_{\mathbf{P} 1}\right), \mathbf{P}\left(\mathcal{O}_{\mathbf{P} 1}(-m)\right.\right.$ $\left.\left.\oplus \mathcal{O}_{\mathbf{P} 1}(-n) \oplus \mathcal{O}_{\mathbf{P}_{1}}\right)\right)$.

Proof. It follows from Lemma (8.16), Lemma (8.17), Lemma (8.18), Lemma (8.19) and Mori [Mo] that if $(G, X) \in \mathscr{C}(\mathrm{J} 9 ; m, n)$ with $\rho(X) \geqq 4$, thən we can find an equivariant blow-down $(G, Y) \in \mathscr{C}(\mathrm{J} 9 ; m, n)$ with $\rho(Y)=$ $\rho(X)-1$. By construction the (Aut $\left.{ }^{0} F_{m, n}^{k}, \mathrm{~F}_{m, n}^{k}\right)$ 's are relatively minimal under 
the condition on $m, n, \ell, k$ of (1) and (2) (cf. (8.8)). Therefore in view of Lemma (8.17) and Lemma (8.19), it remains to show: Let $(G, X) \in \mathscr{C}$ (J9; $m, n)$ such that by an equivariant morphism $(G, X) \rightarrow\left(G, \mathrm{~F}_{n}\right)$ compactifying $\left(\right.$ Aut $\left.^{0} \mathrm{~F}_{m, n}^{\prime}, \mathrm{F}_{m, n}^{\prime}\right) \rightarrow\left(\right.$ Aut $\left.^{0} \mathrm{~F}_{m, n}^{\prime}, \mathrm{F}_{n}^{\prime}\right), X$ is a $\mathbf{P}^{1}$-bundle over $\mathrm{F}_{n}$, then $(G, X)$ is isomorphic to (Aut ${ }^{0} \mathrm{~F}_{m, n}^{k}, \mathrm{~F}_{m, n}^{k}$ ), $k \geqq \ell$. Since the Brauer group of $\mathrm{F}_{n}$ vanishes, there exists a vector bundle $E$ of rank 2 such that $X$ is $\mathrm{F}_{n^{-}}$isomorphic to $\mathrm{P}(E)$. The restriction of $E$ over $\mathrm{F}_{n}^{\prime}=\mathrm{F}_{n}-C_{\infty}$ is denoted by $E^{\prime}$. Then it follows from the argument of the Proof of Lemma (8.17) that $\mathbf{P}\left(E^{\prime}\right)$ is the natural relative completion of $\mathbf{A}^{1}$-bundle $\mathrm{F}_{m, n}^{\prime} \rightarrow \mathrm{F}_{n}^{\prime}$ over $\mathrm{F}_{n}^{\prime}$ hence we may assume $E^{\prime} \cong \mathcal{O}_{\mathrm{F}_{n}^{\prime}} \oplus \mathcal{O}_{\mathrm{F}_{n}^{\prime}}(-m)$. The $\mathbf{P}^{1}$-bundle $\mathbf{P}\left(E^{\prime}\right) / \mathrm{F}_{n}^{\prime}$ has an equivariant section $s^{\prime}: \mathrm{F}_{n}^{\prime} \rightarrow \mathbf{P}\left(E^{\prime}\right)$ corresponding to the projection $\mathcal{O}_{\mathrm{F}_{n}^{\prime}} \oplus \mathcal{O}_{\mathrm{F}_{n}^{\prime}}(-m) \rightarrow \mathcal{O}_{\mathrm{F}_{n}^{\prime}}(-m)$. In other words $s^{\prime}$ is an equivariant rational section $s: \mathrm{F}_{n} \rightarrow \mathbf{P}(E)$. Since a rational map to a complete variety is regular in codimension $1, s$ is $G$-equivariant and $C_{\infty}=\mathrm{F}_{n}-\mathrm{F}_{n}^{\prime}$ is a $G$ orbit, $s$ is an equivariant section of $\mathrm{P}(E) / \mathrm{F}_{n}$ extending $s^{\prime}$. Therefore we can find a line bundles $L, M$ on $\mathrm{F}_{n}$ such that (1) $M \mid \mathrm{F}_{n}^{\prime} \cong \mathcal{O}_{\mathrm{F}_{n}^{\prime}}(m)$ and (2) $E$ is an extension: $\left(^{*}\right) 0 \rightarrow L \rightarrow E \rightarrow M \rightarrow 0$. Since by definition of $s L \mid \mathrm{F}_{n}^{\prime} \cong$ $\mathcal{O}_{F_{h}^{\prime}}$, we may assume $L \cong \mathcal{O}_{\mathrm{F}_{n}}$ by tensoring $L^{-1}$ if necessary. The first condition implies $M \cong \mathcal{O}_{\mathrm{F}_{n}}(-m) \otimes \mathcal{O}_{\mathrm{F}_{n}}\left(-j C_{\infty}\right)$. It follows from the proof of Lemma (8.1) $j \geqq \ell$. Let us show that the extension is trivial; $E \cong L \oplus M$. The extension (*) is parametrized by $H^{1}\left(\mathrm{~F}_{n}, M^{-1}\right)$ and by the restriction map (**) $H^{1}\left(\mathrm{~F}_{n}, M^{-1}\right) \rightarrow H^{-1}\left(\mathrm{~F}_{n}^{\prime}, M^{-1}\right) \cong H^{1}\left(\mathrm{~F}_{n}^{\prime}, \mathcal{O}_{F_{n}^{\prime}}(m)\right)$, the class [E] map to 0 . Now the triviality of the class $[E]$ follows from the following diagram that shows the injectivity of the restriction map (***).

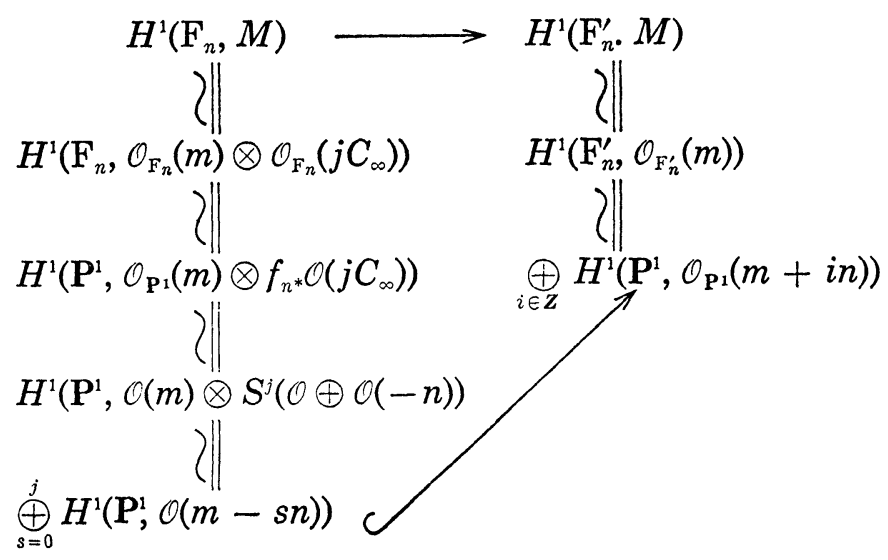

(cf. (1.2.1) and (1.3.1)). 
Remark (8.20.1). We can prove Theorem (8.20) without using [Mo]. To this end we associate to a (successive) equivariant blow up of $\mathrm{F}_{m, n}^{e}$ a diagram. We define inductively the diagram whose set of vertices consists of the irreducible invariant divisors. The diagram is composed of vertices, an edge and arrows joining vertices. Each vertex is painted white or black. Namely, we associate to $\mathrm{F}_{m, n}^{e}$ a diagram consisting of 2-white vertices representing the invariant divisors $D_{1}^{e}, D_{2}^{e}$ and connect them by an edge.

$$
\mathrm{F}_{n} \odot \mathrm{F}_{m-\imath n}
$$

We add to this diagram the intersection number of the divisor with its fibre. For example, on the vertex $\mathrm{F}_{n}$, we associate the intersection number $\left(\mathrm{F}_{n} \cdot f\right)=-\ell$, where $f$ denotes a fibre of the $\mathbf{P}^{1}$-bundle $\mathrm{F}_{n} \rightarrow \mathbf{P}^{1}$. Therefore the number $\left(\mathrm{F}_{m-\ell n} . f\right)=0$ is associated to $\mathrm{F}_{m-\ell n}$. Our diagram for $\mathrm{F}_{n, n}^{\ell}$ is

$$
\begin{array}{cc}
\mathrm{F}_{n} \multimap & \mathrm{F}_{m-\ell n} \\
-\ell & 0 .
\end{array}
$$

Now we assume that a diagram is associated with a (successive) blowup $Y$ of $\mathrm{F}_{m, n}^{e}$ and we define a diagram for an equivariant blow-up $\pi: X \rightarrow Y$. The diagram for $X$ is defined to be the union of the diagram for $Y$ and a vertex which represents the exceptional divisor $E$ for $\pi$ so that set of the vertices of the diagram for $X$ is considered as the set of the irreducible invariant divisors on $X$. The colour of the new vertex corresponding to the exceptional divisor $E$ is white if the center of the blow-up $\pi$ lies on the proper transform $\mathrm{F}_{n}$ of $D_{2}^{\ell}$ on $\mathrm{F}_{m, n}^{\ell}$ and otherwise black. We join a vertex corresponding to a divisor $D$ on $Y$ with the vertex $E$ by an arrow pointing to $E$ if the center of the equivariant blow up is on $D$. We write at each vertex the intersection number of the corresponding divisor with its fibre. This is easily done by the following rule: (1) Write -1 for the new exceptional divisor $E$; (2) For vertices to be connected with the vertex $E$, diminish by 1 from the number for $Y$; (3) Write the same number for other vertices coming from $Y$.

Here are some examples. For $X_{1}$ in (8.7) we have

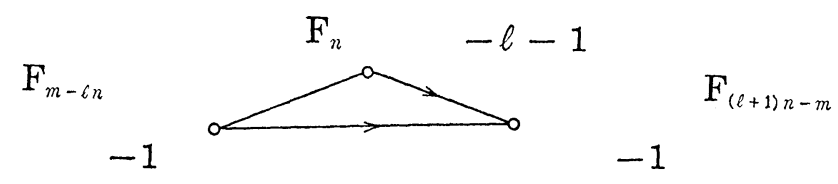


If we blow up $X_{1}$ along $\mathrm{F}_{n} \cap \mathrm{F}_{(\ell+1) n-m}$, the diagram is

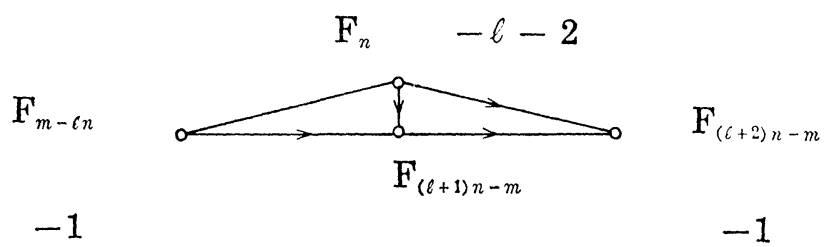

here $\mathrm{F}_{(\ell+2) n-m}$ is the esceptional divisor (c.f (8.7)).

If we blow up $X_{1}$ along $\mathrm{F}_{m-\ell n} \cap \mathrm{F}_{(\ell+1) n-m}$, then the diagram is

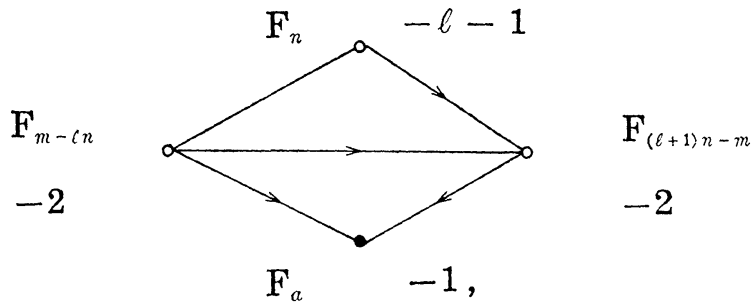

where $\mathrm{F}_{a}$ is the exceptional divisor. We know

$$
a= \begin{cases}(2 \ell+1) n-2 m & \text { if }(2 \ell+1) n-2 m \geq 0, \\ 2 m-(2 \ell+1) n & \text { if }(2 \ell+1) n-2 m<0,\end{cases}
$$

by Lemma (8.5.4).

In (8.20.2) if we blow up along $\mathrm{F}_{(\ell+1) n-m} \cap \mathrm{F}_{a}$, we get

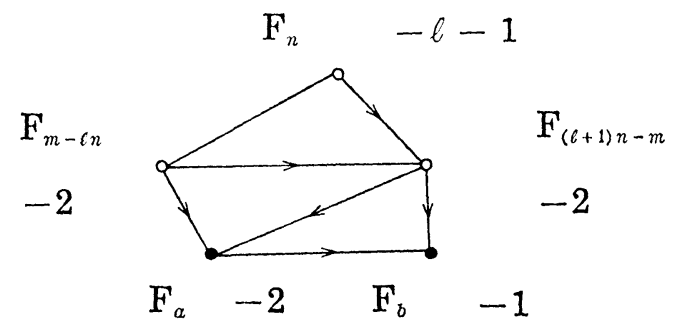

We can determine $b$ by Lemma (8.5.4).

The set of vertices or the invariant divisors is an ordered set by the arrows. Namely let $A, B$ be vertices for $X$. Then we define $A>B$ if there is an arrow from $A$ to $B$. We say that an element $x$ of an ordered set $s$ is relatively minimal if there is no element $y \in S$ such that $x>y$. We conclude by Corollary (8.15).

Proposition (8.20.3). We assume $(m, n) \geq 2$. Let $Y$ be an equivariant blow-up of $\mathrm{F}_{m, n}^{\ell}$ and $D$ be an irreducible invariant divisor on $Y$. Then 
(A) the diagram for $Y$ is connected and (B) the following conditions are equivalent.

(1) The divisor $D$ can be equivariantly blown down giving a nonsingular projective threefold.

(2) In the diagram of $Y$ the number associated with the divisor $D$ is -1 .

If the divisor $D$ corresponds to a black vertex, then the conditions (1), (2) are equivalent to the following condition.

(3) The vertex corresponding to $D$ is relatively minimal.

(C) Let $x$ be a black vertex. Then any vertex $y$ with $x>y$ is black.

Proof. The assertion follows from Corollary (8.15) by calculating the intersection number. Notice that $Y$ is a successive equivariant blow-up with centers isomorphic to $\mathbf{P}^{1}$.

Let $\tilde{X}$ be a (successive) equivariant blow-up of $\mathrm{F}_{m, n}^{\ell}$. Let us study a successive equivariant blow-down of $\tilde{X} ; \tilde{X} \rightarrow Y_{1} \rightarrow Y_{2} \rightarrow \cdots Y_{s}$, where we assume that the $Y_{i}$ 's are projective and non-singular. Let us first study $p_{1}: \tilde{X} \rightarrow Y$. Thus the exceptional divisor for $p_{1}$ is a vertex of the diagram for $\tilde{X}$ with intersection number -1 . We associate to $Y_{1}$ a diagram, whose vertices are the invariant divisors on $Y_{1}$. We write for each vertex the intersection number similarly as in $\tilde{X}$. We explain how we join the vertices. We construct the diagram for $Y_{1}$ from that of $\tilde{X}$ as follows. We eliminate from the diagram for $\tilde{X}$ the vertex of the exceptional divisor for $p_{1}$, arrows and edge related with this vertex. The number for a remaining vertex is unchanged if the corresponding divisor on $\tilde{X}$ is disjoint from the exceptional divisor for $p_{1}$ and otherwise the number is increased by 1 . For the remaining vertices, we keep arrows and colours unchanged. The diagram for $Y_{1}$ has the following properties.

(8.20.4) (1) The diagram is connected.

(2) The vertices consist of the invariant divisors.

(3) For an invariant divisor, the following conditions are equivalent.

(i) The divisor is exceptional and can be equivariantly blown down on a projective non-singular threefold.

(ii) The number associated with the vertex is -1 .

If the vertex is black, these conditions are equivalent to the following.

(iii) The vertex is relatively minimal.

(4) Let $x$ be a black vertex. Then any vertex $y$ with $x>y$ is black. 
Now for $p_{2}: Y_{1} \rightarrow Y_{2}$, we can argue similarly and define a diagram for $Y_{2}$. The diagram for $Y_{2}$ has the properties (8.20.8). Inductively we define a diagram for $Y_{s}$ and the diagram for $Y_{s}$ has the properties (8.20.4) too.

If the diagram for $Y_{s}$ has a black vertex, it follows from the properties (4) and (3.iii) of (8.20.4) that we can find a black-vertex which can be blown down. We can find an equivariant blown down $Y_{s} \rightarrow Y$ such that the diagram for $Y$ consists only of white vertices and then the diagram for $Y$ is one of the following:

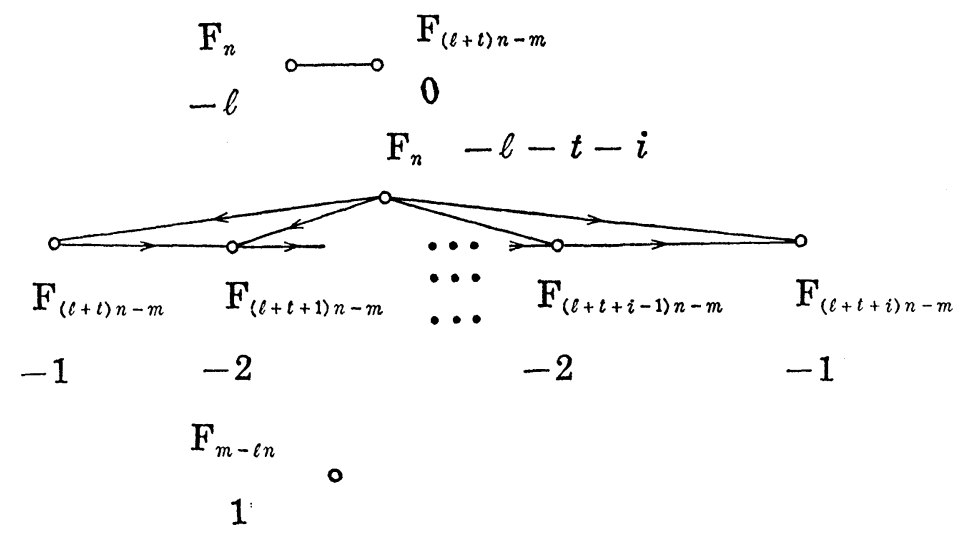

In other words $Y$ and hence $Y_{s}$ is an equivariant blow-up of $\mathrm{F}_{m, n}^{\ell+t}$ in case of the first and second diagram. The case of the third diagram occurs when $\ell=1$ and in this case $Y_{s}$ is an equivariant blow up of $\mathbf{P}\left(\mathcal{O}_{\mathbf{P} 1}(-m) \oplus \mathcal{O}_{\mathbf{P} 1}(-n) \oplus \mathcal{O}_{\mathbf{P} 1}\right)$.

Now let us treat the case $(m, n)=1$. First we give an example to illustrate the situation.

Example (8.21). $m=3, n=2$ and $\ell=1, r=1$. On $\mathrm{F}_{3,2}$ we have isomorphisms $D_{2}^{1} \simeq \mathrm{F}_{2}, D_{1}^{1} \simeq \mathrm{F}_{1}$. In terms of the graph,

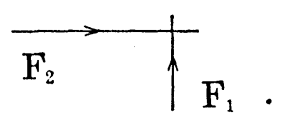

If we blow up $\mathrm{F}_{3,2}^{1}$ along $\mathrm{F}_{1} \cap \mathrm{F}_{2}$, we get $\pi_{1}: X_{1} \rightarrow \mathrm{F}_{3,2}^{1}$ with

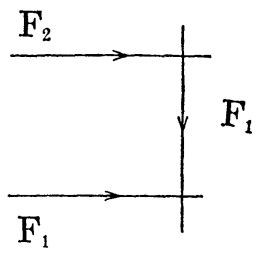


as we have seen in (8.7).

We blow up $X_{1}$ along $\mathrm{F}_{1} \cap \mathrm{F}_{1}$ to get $\pi_{2}: X_{2} \rightarrow X_{1}$. On $X_{2}$ we have

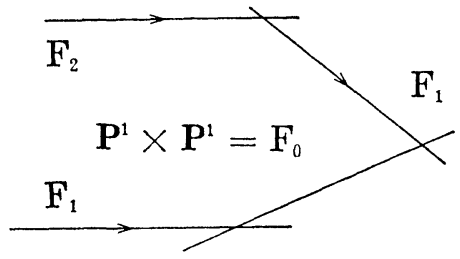

We show that we can collaspe the divisor $F_{0}$ to another direction to get a projective non-singular threefold $U_{3,2}$ which we call the Euclidean model of the operation (Aut ${ }^{0} \mathrm{~F}_{3,2}^{\prime}, \mathrm{F}_{3,2}^{\prime}$ ). Namely there exists a projective non-singular threefold $U_{3,2}$ which is an equivariant completion of (Aut ${ }^{0} \mathrm{~F}_{3,2}^{\prime}, \mathrm{F}_{3,2}^{\prime}$ ) and an equivariant morphism $\pi: X_{2} \rightarrow U_{3,2}$. The morphism $\pi$ is an equivariant blow-up morphism $\pi: X_{2} \rightarrow U_{3,2}$. The morphism $\pi$ is an equivariant blow-up with center $\mathbf{P}^{1}$ and the divisor $F_{0}=\mathbf{P}^{1} \times \mathbf{P}^{1}$ on $X_{2}$ is the exceptional divisor for $\pi$. The restrictions $\pi_{2} \mid \mathrm{F}_{0}$ and $\pi \mid \mathrm{F}_{0}$ are equivalent to different projections; $a \times \mathbf{P}^{1} \subset \mathbf{P}^{1} \times \mathbf{P}^{1}$ is collapsed by one of the morphisms $\pi_{2}$ and $\pi$, and is mapped isomorphically by the other for any $a \in P$. By calculating the intersection number we know that we can contract analytically the divisor $\mathrm{F}_{0}$ for a different direction. However the result of Mori [Mo] tells us that we can do it algebraically. In fact for this purpose we show that the cone $N E(X)$ is polyhedral. Precisely the cone $N E\left(X_{2}\right)$ is spanned by a finite number of elements of $N E\left(X_{2}\right)$. We determine even a linearly independent generators of the cone $N E\left(X_{2}\right)$. The argument works not only for $X_{2}$ but also for any equivariant blow up of $\mathrm{F}_{3,2}^{1}$. To begin with, we show that the cone $N E\left(\mathrm{~F}_{3,2}^{1}\right)$ is spanned by the fibres of $D_{2}^{1} \simeq \mathrm{F}_{2}$ and $D_{1}^{1} \simeq \mathrm{F}_{1}$ and by the curve $\mathrm{F}_{1} \cap \mathrm{F}_{2}$. In fact let $\mathbf{G}_{a} \subset \mathrm{SL}_{2}$ be a subgroup of $\mathrm{SL}_{2}$ isomorphic to $\mathbf{G}_{a}$. since a semi-simple part of Aut $^{0} \mathrm{~F}_{3,2}^{\prime}$ is $\mathrm{SL}_{2}$, we can consider $\mathbf{G}_{a} \subset \mathrm{SL}_{2}$ as a one dimensional subgroup of Aut $^{0} \mathrm{~F}_{3,2}^{\prime}$. We have a $\mathbf{G}_{a}$-equivariant morphism

$$
f_{2} \circ f_{3,2}^{1}: \mathrm{F}_{3,2} \longrightarrow \mathbf{P}^{1} \text {. }
$$

The subgroup $\mathbf{G}_{a}$ has an open orbit $\mathbf{A}^{1} \subset \mathbf{P}^{1} . \quad \mathrm{F}_{3,2}^{\prime}$ is trivial over $\mathbf{A}^{1} ; \mathrm{F}_{3,2}^{\prime} \cap$ $\left(f_{2} \circ f_{3,2}^{\prime}\right)^{-1} \mathbf{A}^{1} \simeq \mathbf{A}^{3}$ making the following diagram commutative

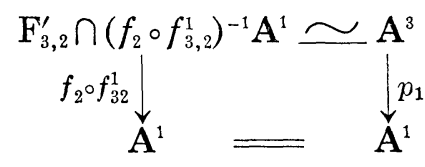


We may assume that the operation of $\mathbf{G}_{a}$ on $\mathbf{A}^{3}$ is $(x, y, z) \rightarrow(x+a, y, z)$ (cf. [U4] p. 396). We show that $C$ is rationally equivalent to an effective curve on $F_{1} \cup F_{2}$ and hence the cone $N E\left(\mathrm{~F}_{3,2}^{1}\right)$ is spanned by the fibres of $D_{2}^{1} \simeq \mathrm{F}_{2}$ and $D_{1}^{1} \simeq \mathrm{F}_{1}$ and by $D_{1}^{1} \cap D_{2}^{1}$ since in general an effective curve on the ruled surface $\mathrm{F}_{a}(a \geq 0)$ is linearly equivariant to a non-negative linear combination $m f+n S$ where $f$ is the fibre of $\mathrm{F}_{a}$ and $S$ is the section of the ruled surface $\mathrm{F}_{a} \rightarrow \mathbf{P}^{1}$ with $S^{2}=-a$.

If the curve $C$ is not contained in $\mathrm{F}_{3,2}^{1}-\mathbf{A}^{3}$ and if $C \cap \mathbf{A}^{3}$ is not $\mathbf{G}_{a^{-}}$invariant (or equivalently $C \cap \mathbf{A}^{3}$ is not of the form $\left\{(x, a, b) \in \mathbf{A}^{3} \mid x \in \mathbf{A}^{1}\right\}$ for a certain point $\left.(a, b) \in \mathbf{A}^{2}\right)$, then using the operation of $\mathbf{G}_{a}$, the curve $C$ is rationally equivalent to an effective curve over $\mathrm{F}_{3,2}-\mathbf{A}^{3}$. For any curve on $\mathrm{F}_{3,2}^{1}-\mathbf{A}^{3}$ or for any curve on $\mathbf{A}^{3}$ excluded above, using operations of the additive group $\mathbf{G}_{a}$ along the fibres of $\mathrm{F}_{3,2}^{1} \rightarrow P^{1}$, we conclude that they are rationally equivalent to effective curves on $D_{1}^{1} \cup D_{2}^{1}$. We proved that the cone $N E\left(\mathrm{~F}_{3,2}^{1}\right)$ is spanned by the fibres of $D_{2}^{1} \simeq \mathrm{F}_{2}$ and $D_{1}^{1} \simeq \mathrm{F}_{1}$ and by $D_{1}^{1} \cap D_{2}^{1}$. Since the Picard number of $\mathrm{F}_{3,2}^{1}$ is $3, \operatorname{dim} N\left(\mathrm{~F}_{3,2}^{1}\right)$ $=3$ and hence the 1-cycles the fibres of $D_{1}^{1}$ and $D_{2}^{1}$ and $D_{1}^{1} \cap D_{2}^{1}$ are linearly independent and therefore they are edges of $N E\left(\mathrm{~F}_{3,2}^{1}\right)$. The same argument works for any equivariant blow-up of $\mathrm{F}_{3,2}^{1}$. For example for $X_{1}$ any effective 1-cycle on $X_{1}$ is rationally equivalent to an effective 1-cycle on $\mathrm{F}_{2} \cup \mathrm{F}_{1} \cup \mathrm{F}_{1}$. Therefore $N E\left(X_{1}\right)$ is spanned by $\mathrm{F}_{2} \cap \mathrm{F}_{1}, \mathrm{~F}_{1} \cap \mathrm{F}_{1}$ and by the fibres of $F_{2}$ and the $F_{1}$ 's. As we noticed above, any effective divisor on $\mathrm{F}_{a}$ is linearly equivalent to a non-negative linear combination $m f+n S$ and hence the 1-cycle $F_{2} \cap F_{1}$ is numerically equivalent to a non-negative linear combination of 1-cycles $F_{1} \cap F_{1}$ and a fibre of the exceptional divisor $\mathrm{F}_{1}$. Therefore the cone $N E\left(X_{1}\right)$ is spanned by $\mathrm{F}_{1} \cap \mathrm{F}_{1}$ and fibres of $\mathrm{F}_{2}$ and the $\mathrm{F}_{1}$ 's. They are linearly independent and they are the edges of $N E\left(X_{1}\right)$. For $X_{2}$, we conclude that $N E\left(X_{2}\right)$ is spanned by $\mathrm{F}_{1} \cap \mathrm{F}_{0}$ and fibres of $F_{2}, F_{1}, F_{0}$ and $F_{1}$, here in the intersection $F_{1} \cap F_{0}$ we may take any one of the $F_{1}$ 's. By the adjunction formula the canonical bundle of $F_{0}$ is $\left(\mathrm{F}_{0}+K_{X_{2}}\right) \mid \mathrm{F}_{0}$. Using again the adjunction formula for the curve $\mathrm{F}_{0} \cap \mathrm{F}_{1}=\ell$ we get $\left(\left(\left(\mathrm{F}_{0}+K_{X_{2}}\right) \mid \mathrm{F}_{0}\right)+\ell \cdot \ell\right)_{\mathrm{F}_{0}}=-2$. On the other hand $\left(\left(\left(\mathrm{F}_{0}+K_{X_{2}}\right) \mid \mathrm{F}_{0}\right)+\ell \cdot \ell\right)_{\mathrm{F}_{0}}=\left(\left(\left(\mathrm{F}_{0}+K_{X_{2}}+\mathrm{F}_{1}\right) \mathrm{F}_{0} \cdot \mathrm{F}_{1} \cdot \mathrm{F}_{0}\right)_{\mathrm{F}_{0}}=\left(\mathrm{F}_{0}+K_{X_{2}}+\mathrm{F}_{1} \cdot \mathrm{F}_{1}\right.\right.$. $\left.\mathrm{F}_{0}\right)=\left(\mathrm{F}_{0} \cdot \mathrm{F}_{1} \cdot \mathrm{F}_{0}\right)+\left(K_{X_{2}} \cdot \mathrm{F}_{1} \cdot \mathrm{F}_{0}\right)+\left(\mathrm{F}_{1} \cdot \mathrm{F}_{1} \cdot \mathrm{F}_{0}\right)=-1+\left(K_{X_{2}} \cdot \mathrm{F}_{1} \cdot \mathrm{F}_{0}\right)$. Therefore $\left(K_{X_{2}} \cdot \ell\right)=-1$ and $\ell=\mathrm{F}_{1} \cap \mathrm{F}_{0}$ is an extremal rational curve. It follows from Lemmas (8.16) and (8.17) and from [Mo] that we can contract $F_{0}$ to another direction; $\pi ; X_{2} \rightarrow U_{3,2}$ we have 


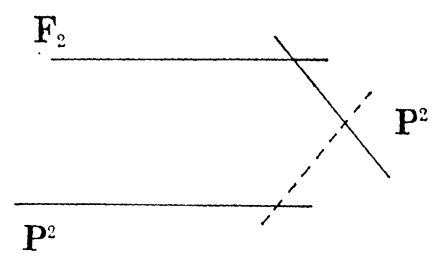

where the doted line represents $\mathbf{P}^{1}$ consisting of the fixed points. Since $\left(\mathrm{F}_{2} . \mathrm{F}_{0} . \mathrm{F}_{0}\right)=-2$, we get $\mathcal{O}\left(\mathbf{P}^{2}\right) \otimes \mathcal{O}_{\mathbf{P}^{2}} \simeq \mathcal{O}_{\mathbf{P}_{2}}(-2)$ for $\mathbf{P}^{2}$ intersecting $\mathrm{F}_{2}$. For another $\mathrm{P}^{2}$, we have over $\mathrm{F}_{3,2}^{1}, \quad \mathcal{O}\left(\mathrm{F}_{1}\right) \otimes \mathcal{O}_{\mathrm{F}_{1}}=p^{*} \mathcal{O}_{\mathrm{P}_{1}}(-2)$ since $\mathrm{F}_{1}=p^{-1} C_{2}$ and $C^{2}=-2$. There is a line on this $\mathbf{P}^{2}$ which is isomorphically mapped to a zero section of $\mathrm{F}_{1}$ on $\mathrm{F}_{3,2}^{1}$ and hence we have $\mathcal{O}\left(\mathbf{P}^{2}\right) \otimes \mathcal{O}_{\mathbf{p}^{2}} \simeq \mathcal{O}_{\mathbf{p}^{2}}(-2)$ too. Therefore we can not equivariantly contract none of the divisors on $U_{3,2}$ onto a non-singular variety. Namely (Aut ${ }^{0} \mathrm{~F}_{3,2}^{\prime} . U_{3,2}$ ) is a relatively minimal element of $\mathscr{C}(\mathrm{J} ; 3,2)$. It follows from Lemma (8.5.4) and from the argument of (8.12) that for an equivariant blow-up of $X_{2}$ there is only one invariant divisor isomorphic to $\mathbf{P}^{1} \times \mathbf{P}^{1}$ and there is no invariant $\mathbf{P}^{2}$.

We can generalize Example (8.21) for $\mathscr{C}(\mathrm{J} 9 ; m, n), m>n \geq 2$ with $(m, n)=1$. In fact, we blow up $\mathrm{F}_{m, n}^{\ell}$ along $D_{1}^{\ell} \cap D_{2}^{\ell}$, we get $\pi_{1}: X_{1} \rightarrow \mathrm{F}_{m, n}^{\ell}$ as in (8.7);

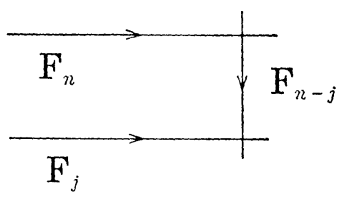

where we put $j=m-\ell$ so that $(n, j)=1$. We blow up $X_{1}$ at $\mathrm{F}_{(\ell+1) n-m}$ $\cap \mathrm{F}_{m-\ell n}$ to get $\pi_{2}: X_{2} \rightarrow X_{1}$. We have on $X_{2}$

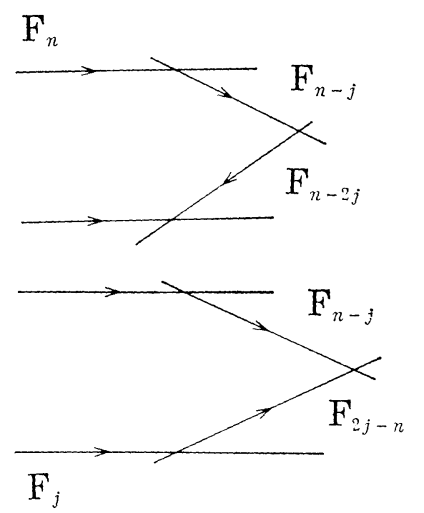

$$
\text { if } n-2 j \geqslant 0
$$

$$
\text { if } 2 j-n>0
$$


Continuing blowing up along the unique curve where the two arrows gather, we arrive by the Euclidean algorithm at the exceptional divisor $\mathrm{F}_{1}$ on $X_{r}$ for some $r$. We have on $X_{r}$

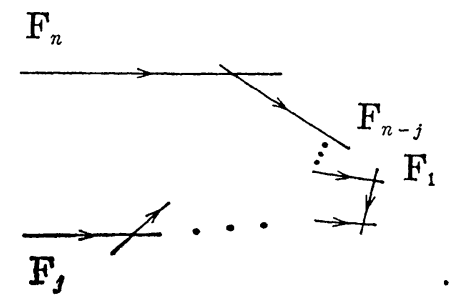

Now we still continue blowing up $X_{r}$ to get finally $X_{s}$

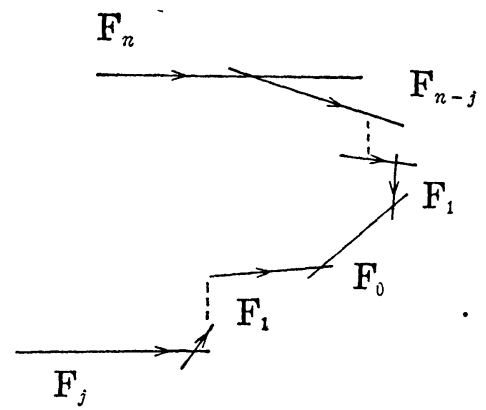

The argument in Example (8.21) shows that (i) an effective 1-cycle on $X_{s}$ is rationally equivalent to an effective 1-cycle on $F_{n} \cup F_{n-j} \cup \cdots \cup$ $F_{1} \cup F_{0} \cup F_{1} \cup \cdots \cup F_{j}$, (ii) $N E\left(X_{s}\right)$ is spanned by the fibres of $F_{n}, F_{n-j}, \cdots$, $F_{1}, F_{0}, F_{1}, \cdots$ and $F_{j}$ and by the 1-cycle $F_{0} \cap F_{1}$, (ii) these 1-cycles are linearly independent and that (iv) they are the edges of the cone $N E\left(X_{s}\right)$. The same calculation as in Example (8.21) shows $\left(K_{X s} \cdot F_{0} . F_{1}\right)=-1$ so that $F_{0} \cap F_{1}$ is an extremal rational curve. As in Example (8.21) we can contract equivariantly $F_{0}$ to another direction to get a projective nonsingular threefold $U_{m n}$ on which the algebraic group Aut ${ }^{0} \mathrm{~F}_{m, n}^{\prime}$ acts. We call $U_{m, n}$ the Euclidean model of $\left(\operatorname{Aut}^{0} \mathrm{~F}_{m, n}^{\prime}, \mathrm{F}_{m, n}^{\prime}\right)$. For any (successive) equivariant blow-up of $X_{s}$, there is only one invariant $\mathrm{F}_{0}$ by Lemma (8.5.4) and there is no invariant $\mathbf{P}^{2}$. As in Example (8.21), we can show that ( Aut $^{0} U_{m, n}, U_{m, n}$ ) is relatively minimal in $\mathscr{C}(\mathrm{J} 9 ; m, n)$.

We can state in a form of Theorem.

Theorem (8.22). We assume $(m, n)=1$. Any element of $\mathscr{C}(\mathrm{J} 9 ; m, n)$ $(m>n \geq 2)$ can be equivariantly blown down to a relatively minimal element of the ordered set $\mathscr{C}(\mathrm{J} 9 ; m, n)$. 
(1) If $m \geq 2 n,(m, n)=1$, then the relatively minimal elements in $\mathscr{C}(\mathrm{J} 9 ; m, n)$ are the $\left(\right.$ Aut $\left.^{0} \mathrm{~F}_{m}^{k}, \mathrm{~F}_{m, n}^{k}\right)$ 's $(k \geq \ell)$ and the Euclidean model (Aut ${ }^{0} U_{m, n}, U_{m, n}$ ).

(2) If $2 n>m,(m, n)=1$, then the relatively minimal elements in $\mathscr{C}(\mathrm{J} 9 ; m, n)$ are the $\left(\operatorname{Aut}^{0} \mathrm{~F}_{m, n}^{k}, \mathrm{~F}_{m, n}^{k}\right)$ 's $(k \geq \ell), \quad\left(\operatorname{Aut}^{0} \mathbf{P}\left(\mathcal{O}_{\mathbf{P} 1}(-m) \oplus \mathcal{O}_{\mathbf{P} 1}(-n)\right.\right.$ $\left.\left.\left.\oplus \mathcal{O}_{\mathbf{P}}\right)\right), \mathbf{P}\left(\mathcal{O}_{\mathbf{P} 1}(-m) \oplus \mathcal{O}_{\mathbf{P} 1}(-n) \oplus \mathcal{O}_{\mathbf{P}_{1}}\right)\right)$ and the Euclidean model (Aut ${ }^{0} U_{m, n}$, $\left.U_{m, n}\right)$.

Proof is done by the same method as in remark (8.20.1). Since there may exist a divisor isomorphic to $\mathbf{P}^{1} \times \mathbf{P}^{1}$ which can be contracted for 2 different directions, in the diagram of invariant divisors we have to associate 2 numbers for $\mathbf{P}^{1} \times \mathbf{P}^{1}$. We do not give here a proof since this is done quite similarly and the explication is messy.

Theorem (8.20) and (8.22) determine the relatively minimal elements of the ordered set $\mathscr{C}(\mathrm{J} 9 ; m, n)$ and we constructed these relatively minimal elements in an explicit way.

Remark (8.23). In the model $U_{3,2}$, we do have the case (3.3.5) of Theorem (3.3) in [Mo].

\section{§9. Equivariant completions of $\mathbf{J 1 0}$}

It follows from Lemma (4.20), [U4] that $\left(\right.$ Aut $^{0} \mathrm{~F}_{m, m}^{\prime}, \mathrm{F}_{m, m}^{\prime}$ ) has an equivariant completion (Aut $\left.{ }^{0} \mathrm{~F}_{m, m}, \mathrm{~F}_{m, m}\right)$, where $\mathrm{F}_{m, m}$ denotes $\mathbf{P}\left(\mathcal{O}_{\mathbf{P} 1}(-m) \oplus\right.$ $\left.\mathcal{O}_{\mathbf{P} 1}(-m) \oplus \mathcal{O}\right)(m \geq 2)$. We put $\mathscr{C}(\mathrm{J} 10 ; m)=\mathscr{C}\left(\right.$ Aut $\left.^{0} \mathrm{~F}_{m, m}^{\prime}, \mathrm{F}_{m}^{\prime}\right)(m \geq 2)$. As in the other preceding cases we want to know the orbit decomposition.

Lemma (9.1). The orbit decomposition of (Aut $\mathrm{F}_{m, m}, \mathrm{~F}_{m, m}$ ) is $\mathrm{F}_{m, m}^{\prime} \mathrm{U}$ $\left(\mathrm{F}_{m, m}-\mathrm{F}_{m, m}^{\prime}\right)$. The latter orbit is a divisor $D$ isomorphic to $\mathbf{P}^{1} \times \mathbf{P}^{1}$. Furthermore $\mathcal{O}_{D}(D) \simeq \mathcal{O}_{\mathbf{P} 1 \times \mathbf{P} 1}(-m, 1)$.

Proof. The first assertion follows from Lemma (4.20), [U4]. Let $U_{0}=\left\{\left(x_{0}, x_{1}\right) \in \mathbf{P}^{1} \mid x_{0} \neq 0\right\}$ and $U_{1}=\left\{\left(x_{0}, x_{1}\right) \in \mathbf{P}^{1} \mid x_{1} \neq 0\right\}$. Then it follows from the definition that $\mathrm{F}_{m, m}$ is defined by gluing together $U_{0} \times \mathbf{P}^{2}$ and $U_{1} \times \mathbf{P}^{2}:\left(\mathrm{s} ;\left(u_{0}, u_{1}, u_{2}\right)\right) \in U_{0} \times \mathbf{P}^{2}$ and $\left(t ;\left(v_{0}, v_{1}, v_{2}\right)\right) \in U_{1} \times \mathbf{P}^{2}$ are identified if $t=1 / s, v_{0}=s^{-m} u_{0}, v_{1}=s^{-m} u_{1}$ and $u_{2}=v_{2}$. $\quad D=\mathrm{F}_{m, m}-\mathrm{F}_{m, m}^{\prime}$ is

$$
\left\{\left(s ;\left(u_{0}, u_{1}, 0\right)\right) \in U_{0} \times \mathbf{P}^{2}\right\} \cup\left\{\left(t ;\left(v_{0}, v_{1}, 0\right)\right) \in U_{1} \times \mathbf{P}^{2}\right\}
$$

hence isomorphic to $\mathbf{P}^{1} \times \mathbf{P}^{1}$. Let $f$ be a fibre of the projection $D \rightarrow \mathbf{P}^{1}$ induced by the map $\mathrm{F}_{m, m} \rightarrow \mathbf{P}^{1}$. Since $f$ is a line in the fibre isomorphic to $\mathrm{P}^{2}$ and $D$ is defined by $u_{2}=v_{2}=0$ inducing the hyperplane section on 
the fibre $\mathbf{P}^{2},(D . f)=1$. To determine the degree for another projection of $D$, we put $\ell=\left\{(s ;(1,0,0)) \in U_{0} \times \mathbf{P}^{2}\right\} \cup\left\{(t ;(1,0,0)) \in U_{1} \times \mathbf{P}^{2}\right\}$ and calculate the intersection number $(D . \ell)$. On $W_{0}=\left\{\left(s ;\left(u_{0}, u_{1}, u_{2}\right)\right) \in U_{0} \times \mathbf{P}^{2} \mid u_{0} \neq 0\right\}$ $D$ is defined by a regular function $u_{2} / u_{2}=0$ and on $W_{1}=\left\{\left(t ;\left(v_{0}, v_{1}, v_{2}\right) \in\right.\right.$ $\left.U_{1} \times \mathbf{P}^{2} \mid v_{0} \neq 0\right\} D$ is defined by a regular function $v_{2} / v_{0}=0$. Therefore on $W_{0} \cup W_{1}, D$ defines a line bundle whose transition function is $\left(v_{2} / v_{0}\right) /\left(u_{2} / u_{0}\right)$ $=u_{0} / v_{0}=t^{m}$. Thus $\mathcal{O}(D) \otimes \mathcal{O}_{\ell} \simeq \mathcal{O}_{\mathbf{P}}(-m)$.

TheOREm (9.2). The set $\mathscr{C}(\mathrm{J} 10 ; m)(m \geq 2)$ consists of one element $\left(\right.$ Aut $^{0} \mathrm{~F}_{m, m}, \mathrm{~F}_{m, m}$ ).

Proof. Since $\mathcal{O}(D) \otimes \mathcal{O}_{D} \simeq \mathcal{O}_{\mathbf{P} 1 \times \mathbf{P} 1}(-m, 1)$, we can not collapse $D$ neither to a smooth point by Lemma (1.12) nor to a curve. The theorem now follows by the argument of Theorem (3.2).

\section{§10. Equivariant completions of J11}

Let us first construct some small equivariant completions. Let us recall the definition of $\mathrm{E}_{m}^{\prime \ell}$ : using the notations of the section $1 \mathrm{E}_{m}^{\prime \ell}$ is the $\mathbf{A}^{1}$-bundle over $\mathrm{F}_{m}^{\prime}$ defined by the unique non-trivial extension

$$
0 \longrightarrow \mathcal{O}_{\mathrm{F}_{m}^{\prime}} \longrightarrow \mathscr{E} \longrightarrow \mathcal{O}_{\mathrm{F}_{m}^{\prime}}(2-\ell m) \longrightarrow 0
$$

which is homogeneous under the operation of $\mathrm{SL}_{2}$ on $\mathrm{F}_{m}^{\prime}$. We know by [U4] that $\mathrm{J} 11$ is the operation ( $\left.\mathrm{Aut}^{0} \mathrm{E}_{m}^{\prime \ell}, \mathrm{E}_{m}^{\prime l}\right)(m \geq 2, \ell \geq 2$ or $m=1, \ell \geq 3$ ) which respects consequently the natural fibrations $\mathrm{E}_{m}^{\prime \ell} \rightarrow \mathrm{F}_{m}^{\prime} \rightarrow \mathbf{P}^{1}$. The extension $0 \rightarrow \mathcal{O}_{\mathrm{F}_{m}^{\prime}} \rightarrow \mathscr{F} \rightarrow \mathcal{O}_{m}(2-\ell m) \rightarrow 0$ is parametrized by the cohomology group $H^{1}\left(\mathrm{~F}_{m}^{\prime}, \mathcal{O}_{\mathrm{F}_{m}^{\prime}}(\ell m-2)\right)$ which is $\mathrm{SL}_{2}$-isomorphic to $\oplus_{k \geq 0} H^{1}\left(\mathbf{P}^{1}, \mathcal{O}(\ell m-2\right.$ - $k m)$ ) by (1.3.1) and $E_{m}^{\prime}$ corresponds to the unique $\mathrm{SL}_{2}$-invariant subspace $H^{1}\left(\mathbf{P}^{1}, 0(-2)\right)$ of $\oplus_{k \geq 0} H^{1}\left(\mathbf{P}^{1}, \mathcal{O}(\ell m-2-k m)\right.$ ) (see (3.8), [U4]). If we take $j \geq \ell$, then by the degeneracy of the spectral sequence (1.2) of $f: F_{n} \rightarrow \mathbf{P}^{1}$, the extension $\mathscr{E}$ on $\mathrm{F}_{m}^{\prime}$ can be extended over $\mathrm{F}_{m}$ :

$$
0 \longrightarrow \mathcal{O}_{\mathrm{F}_{m}} \longrightarrow \widetilde{\mathscr{E}} \longrightarrow \mathcal{O}_{\mathrm{F}_{m}}(2-\ell m) \otimes \mathcal{O}_{\mathrm{F}_{m}}\left(-j C_{\infty}\right) \longrightarrow 0
$$

where $\mathrm{C}_{\infty}=\mathrm{F}_{m}-\mathrm{F}_{m}^{\prime}$ (see (1.30) and the section 8). The hypotheses $j \geq \ell$ implies $(\ell-j) m-2 \leq 0$ and hence the morphism $H^{0}\left(\mathrm{~F}_{m}, \mathcal{O}_{\mathrm{F}_{m}}(\ell m-2) \otimes\right.$ $\left.\mathcal{O}_{\mathrm{F}_{m}}\left(j C_{\infty}\right)\right) \rightarrow H^{0}\left(\mathrm{~F}_{m}^{\prime}, \mathcal{O}_{\mathrm{F}_{m}^{\prime}}(\ell m-2)\right)$ induced by the restriction is an isomorphism. In fact by the degeneracy of spectral sequences (1.2) and (1.3.1), we have a commutative diagram: 


$$
\begin{aligned}
& H^{0}\left(\mathrm{~F}_{m}, \mathcal{O}_{\mathrm{F}_{m}}(l m-2) \otimes \mathcal{O}_{\mathrm{F}_{m}}\left(j C_{\infty}\right)\right) \longrightarrow H^{0}\left(\mathrm{~F}_{m}^{\prime}, \mathcal{O}_{\mathrm{F}_{m}^{\prime}}(l m-2)\right) \\
& \bigoplus_{i=0}^{j} H^{0}\left(\mathbf{P}^{1}, \mathcal{O}_{\mathbf{P}_{1}}(l m-2) \otimes \mathcal{O}(-i m)\right) \simeq \bigoplus_{i=0}^{\infty} H^{0}\left(\mathbf{P}^{1}, \mathcal{O}_{\mathbf{P}_{1}}(l m-2) \otimes \mathcal{O}(-i m)\right) .
\end{aligned}
$$

Hence the $\mathbf{P}^{1}$-bundle $f_{m}^{\ell}(j): \mathrm{E}_{m}^{\varepsilon}(j)=\mathbf{P}(\widetilde{\mathscr{E}}) \rightarrow \mathrm{F}_{m}$ is equivariant completion of $\left(\mathrm{Aut}^{0} \mathrm{E}_{m}^{\prime \ell}, \mathrm{E}_{m}^{\prime \prime}\right)$ by Lemma (4.22), [U4].

Notation (10.1). We denote by $D_{1}^{\ell}(j)$ a divisor $f_{m}^{\ell}(j)^{-1}\left(C_{\infty}\right)$ and $D_{2}^{\ell}(j)$ the $\infty$-section of the $\mathbf{P}^{1}$-bundle $f_{m}^{\ell}(j): \mathrm{E}_{m}^{\ell}(j) \rightarrow \mathbf{P}^{1}$ arising from the $\mathbf{A}^{1}$-bundle or the extension $(* *)$ so that $\mathrm{E}_{m}^{\ell}(j)=\mathrm{E}_{m}^{\prime \ell} \cup D_{1}^{\ell}(j) \cup D_{2}^{\ell}(j)$.

Lemma (10.2). (a) The divisor $3 D_{1}^{\ell}(j)$ and $D_{2}^{\ell}(j)$ are $\operatorname{Aut}^{0} \mathrm{E}_{m}^{\ell}(j)$-invariant. (b) $\mathrm{E}_{m}^{l}(j)-\left(D_{1}^{\ell}(j) \cup\left(D_{1}^{\ell}(j)\right)\right.$ is an open orbit. (c) $D_{1}^{\ell}(\ell)$ is isomorphic to $\mathbf{P}^{1} \times \mathbf{P}^{1}$ and the Aut ${ }^{0} \mathrm{E}_{m}^{\ell}(\ell)$-orbit decomposition of $D_{1}^{\prime}(\ell) \simeq \mathbf{P}^{1} \times \mathbf{P}^{1}$ is $\left(\mathbf{P}^{1} \times \mathbf{P}^{1}\right.$ - diagonal $) \cup$ diagonal. $\left(\mathrm{c}^{\prime}\right)$ If $j>\ell$, then $D_{1}^{e}(j)$ is isomorphic to $\mathrm{F}_{(j-\ell) m+2}$ and the Aut $\mathrm{E}_{m}^{\ell}(j)$-orbit decomposition of $D_{1}^{\ell}(j) \simeq \mathrm{F}_{(j-\ell) m+2}$ consists of an open orbit and two $\mathbf{P}^{1}$ 's which are disjoint sections of the ruled surface $\mathrm{F}_{(j-\ell) m+2} \rightarrow \mathbf{P}^{1}$ : they are the $\infty$-section and a 0 -section of $\mathrm{F}_{(j-\ell) m+2}$. (d) $L_{2}^{\ell}(j)$ is isomorphic to $\mathrm{F}_{m}$ with orbit decomposition $\left(\mathrm{F}_{m}-C_{\infty}\right) \cup C_{\infty}$.

Proof. The assertions (a), (b) and (d) follow from the construction (cf. the section 8). The restriction to $C_{\infty} \simeq \mathbf{P}^{1}$ of the non-trivial extension $0 \rightarrow \mathcal{O}_{\mathrm{F}_{m}} \rightarrow \mathscr{E} \rightarrow \mathcal{O}_{\mathrm{F}_{m}}(2-\ell m) \otimes \mathcal{O}_{\mathrm{F}_{m}}\left(-\ell C_{\infty}\right) \rightarrow 0$ is an extension:

$$
0 \longrightarrow \mathcal{O}_{\mathbf{P}_{1}} \longrightarrow \mathscr{E} \mid \mathbf{P}^{1} \longrightarrow \mathcal{O}_{\mathbf{P}_{1}}(2-\ell m+\ell m) \longrightarrow 0
$$

since $\left(C_{\infty}\right)^{2}=-m$. We show that the extension (*) is non-trivial. In fact putting $M=\mathcal{O}_{\mathrm{F}_{m}}(-2+\ell m) \otimes \mathcal{O}_{\mathrm{F}_{m}}\left(\ell C_{\infty}\right)$, consider the exact sequence $0 \rightarrow$ $\mathcal{O}_{\mathrm{F}_{m}}\left(-C_{\infty}\right) \rightarrow \mathcal{O}_{\mathrm{F}_{m}} \rightarrow \mathcal{O}_{C_{\infty}} \rightarrow 0$ defining the curve $C_{\infty}$ on $\mathrm{F}_{n}$. Tensoring $M$ with $(*)$, we get $0 \rightarrow \mathcal{O}_{\mathrm{F}_{m}}(-2+\ell m) \otimes \mathcal{O}_{\mathrm{F}_{m}}\left((\ell-1) C_{\infty}\right) \rightarrow M \rightarrow M \otimes \mathcal{O}_{C_{\infty}}\left(\simeq \mathcal{O}_{\mathbf{p}_{1}}(-2)\right)$ $\rightarrow 0$. Hence combining with the spectral sequence for $\mathrm{F}_{m} \rightarrow \mathbf{P}^{1}$ we finally get an $\mathrm{SL}_{2}$-exact sequence:

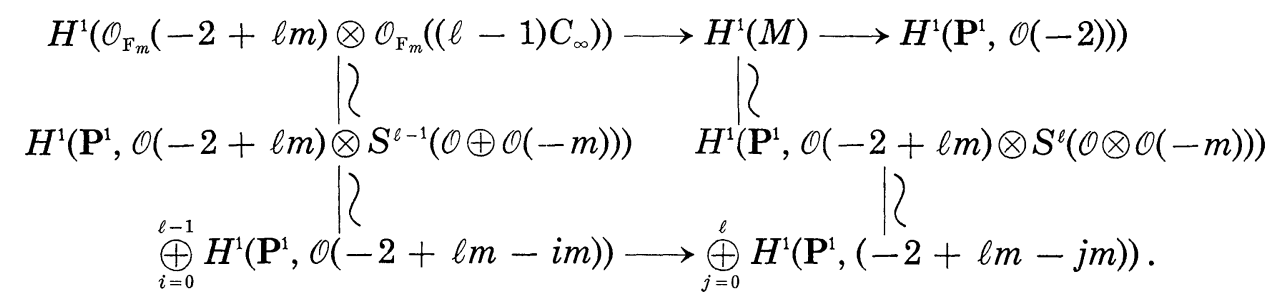

Hence the map $H^{1}(M) \rightarrow H^{1}\left(C_{\infty}, M \otimes \mathcal{O}_{C_{\infty}}\right)$ induces an isomorphism between 
$H^{1}\left(\mathbf{P}^{1}, \mathcal{O}(-2)\right)$-factor in $H^{1}(M)$ and $H^{1}\left(M \otimes \mathcal{O}_{C_{\infty}}\right)$, since $H^{1}\left(\mathbf{P}^{1}, \mathcal{O}(j)\right)$ is the irreducible $\mathrm{SL}_{2}$-module of degree $-1-j$ if $H^{1}\left(\mathbf{P}^{1}, \mathcal{O}(j)\right) \neq 0$. Therefore the exact sequence $(*)$ is $\mathrm{SL}_{2}$-isomorphic to the exact sequence:

$$
0 \longrightarrow \mathcal{O} \longrightarrow \mathcal{O}(1) \oplus \mathcal{O}(2) \longrightarrow \mathcal{O}(2) \longrightarrow 0 \text {. }
$$

Since it follows from the spectral sequence (1.2.1) that the inclusion map

$$
\begin{aligned}
H^{0}\left(\mathrm{~F}_{m}, \mathcal{O}_{\mathrm{F}_{m}}(-2+\ell m) \otimes \mathcal{O}_{\mathrm{F}_{m}}\left((\ell-1) C_{\infty}\right)\right) \\
\longrightarrow H^{0}\left(\mathrm{~F}_{m}, \mathcal{O}_{\mathrm{F}_{m}}(-2+\ell m) \otimes \mathcal{O}_{\mathrm{F}}\left(j C_{\infty}\right)\right)
\end{aligned}
$$

is bijective for any $j \geq \ell$ (cf. the section 8), we conclude from Lmma (1.10) that $\operatorname{Ker} \varphi=H^{0}\left(\mathrm{~F}_{m}, \mathcal{O}_{\mathrm{F}_{m}}(-2+\ell m)\right)$ fixes all the points lying over $C_{\infty}$, where $\varphi$ denotes the morphism Aut $\mathrm{E}_{m}^{\ell}(j) \rightarrow \mathrm{Aut}^{0} \mathrm{~F}_{m}$ induced by the equivariant morphism $\pi$. In particular when $\ell=j$, the action of $\operatorname{Aut}^{0} \mathrm{E}_{m}^{\ell}(\ell)$ on $D_{1} \simeq$ $\mathbf{P}^{1} \times \mathbf{P}^{1}$ is an operation of $\mathrm{SL}_{2}$ on $\mathbf{P}^{1} \times \mathbf{P}^{1}$ respecting the exact sequence (**) and hence the diagonal action of $\mathrm{SL}_{2}$ on $\mathbf{P}^{1} \times \mathbf{P}^{1}$ by Lemma (1.11). This proves (c). Since for $j>\ell$, there is no $\mathrm{SL}_{2}$-invariant in $H^{1}\left(\mathbf{P}^{1}, \mathcal{O}(-2-(j-\ell) m)\right)$, the restriction of the $\mathrm{SL}_{2}$-exact sequence $0 \rightarrow \mathcal{O}_{\mathrm{F}_{m}}$ $\rightarrow \mathscr{E} \rightarrow \mathcal{O}_{\mathrm{F}_{m}}(2-\ell m) \otimes \mathcal{O}_{\mathrm{F}}\left(j C_{\infty}\right) \rightarrow 0$ onto $C_{\infty} \simeq \mathbf{P}^{1} ; 0 \rightarrow \mathcal{O}_{\mathbf{P} 1} \rightarrow \mathscr{E} \otimes \mathcal{O}_{\mathbf{P} 1} \rightarrow \mathcal{O}_{\mathbf{P} 1}(2+$ $(j-\ell) m) \rightarrow 0$ splits and hence $D_{1}^{\ell}(j) \simeq \mathrm{F}_{2+(j-\ell) m}$. $\left(\mathrm{c}^{\prime}\right)$ now follows from isomorphism (10.3), Lemma (1.10), Lemma (1.11) and [U3].

Lemma (10.4). Let $(G, X) \in \mathscr{C}\left(\right.$ Aut $\left.^{0} \mathrm{E}_{m}^{\prime \prime}, \mathrm{E}_{m}^{\prime \prime}\right)=\mathscr{C}(\mathrm{J} 11 ; m, \ell)(m \geq 2, \ell \geq 2$ or $m=1, \ell \geq 3)$. Then there is no $G$-fixed point on $X$ and none of the cases (3.3.2), (3.3.3), (3.3.4) and (3.3.5) of Theorem (3.3) Mori [Mo] occurs.

Proof. A simple part of $G$ is $\mathrm{SL}_{2}$ and it has an open orbit by Corollary (4.23), [U4] and hence there is no $\mathrm{SL}_{2}$-fixed point by Lemma (1.2.2), [MU] and consequently there is no $G$-fixed point on $X$. We show that there is no invariant divisor isomorphic to $\mathbf{P}^{2}$ on $X$. In fact let $\chi: X \cdots \cdots$ $\mathrm{E}_{m}^{\ell}(\ell)$ be an equivariant birational map. Let the diagram

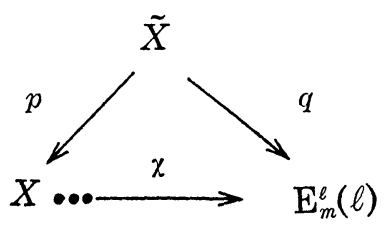

be an equivariant elimination of indeterminancy, where $p$ is an equivariant blow-up. Since there is no fixed point on $\mathrm{E}_{m}^{\ell}(\ell)$, it follows from Theorem (1.7) $q$ is an equivariant blow-up too. Assume that there is an 
invariant $\mathbf{P}^{2}$ on $X$. Since there is no fixed point, the proper transform $p^{-1}\left(\mathbf{P}^{2}\right)$ is isomorphic to $\mathbf{P}^{2}$. This is a contradiction since there is neither invariant $\mathbf{P}^{2}$ nor fixed point on $\mathrm{E}_{m}^{\ell}(\ell)$.

Lemma (10.5). Let $(G, X) \in \mathscr{C}(\mathrm{J} 11 ; m, \ell)(m \geq 2, \ell \geq 2$ or $m=1, \ell \geq 3)$. Let $\phi: X \rightarrow Y$ be the morphism of (3.5.1) Theorem (3.5) of [Mo]. Then the surface is isomorphic to $\mathrm{F}_{m}$ and $X$ is a $\mathbf{P}^{1}$-bundle over $\mathrm{F}_{m}$.

Proof. Since $\operatorname{Aut}^{0}\left(\mathrm{E}_{m}^{\prime \ell} ; \mathrm{F}_{m}\right)=\operatorname{Aut}^{0} \mathrm{E}_{m}^{\prime \ell}$ by Corollary (2.3), [U4], the lemma is proved by the same argument as in the proof of Lemma (8.17).

Lemma (10.6). Let $(G, X) \in \mathscr{C}(\mathrm{J} 11 ; m, \ell) . \quad$ Case (3.5.2) of Theorem (3.5), [Mo] never occurs.

Proof. It follows from Corollary (4.23), [U4] that a semi-simple part $\mathrm{SL}_{2}$ has an open orbit on $X$. Therefore if $\Phi: X \rightarrow Y$ is a morphism of case (3.5.2) of Theorem (3.5), in [Mo], $\mathrm{SL}_{2}$ has an open orbit and we have a non-trivial morphism $(\psi, \phi):(G, X) \rightarrow\left(\mathrm{PGL}_{2}, \mathrm{P}^{1}\right)$. Hence all the fibres of $\phi$ are isomorphic to one non-singular del Pezzo surface $S$ and $S$ is an equivariant completion of a homogeneous surface $Y^{\prime}$ under the unipotent radical $U$ of $G$ since $U$ has an open orbit on each fibre (see [U4]). The homogeneous surface $\left(U, Y^{\prime}\right)$ contains $\left(\mathbf{G}_{a}^{\oplus^{2}}, \mathbf{G}_{a}^{\oplus^{2}}\right)$ since any homogeneous surface under unipotent group contains such operation. It follows now from Theorem (3.5), [Mo] and from Theorem 2.4.4, [Ma] that $S$ is isomorphic to $\mathbf{P}^{1} \times \mathbf{P}^{1}$ or $\mathbf{P}^{2}$. In the last case, there exists a vector bundle $\mathscr{E}$ of rank 3 on $\mathbf{P}^{2}$ such that $X \simeq \mathbf{P}(\mathscr{E})$. By a theorem of Grothendieck we can find 2 line bundles $L, M$ on $\mathbf{P}^{1}$ such that $X \simeq \mathbf{P}(L \oplus M \oplus 0)$. Therefore $\mathbf{G}_{m}^{3}$ operates on $X$ and $\mathbf{G}_{m}^{3} \subset G$. This is absurd. We notice that the rank of $G$ is equal to 2 (see [U4]). Now we exclude the first case $S \simeq \mathbf{P}^{1} \times \mathbf{P}^{1}$. In fact assume that this is the case. We know that a semi-simple part of $G$, which is isogeneous to $\mathrm{SL}_{2}$, has an open orbit (cf. Corollary (4.23), [U4]). Let $B$ be a Borel subgroup of $\mathrm{SL}_{2}$. $B$ has an open orbit on $\mathbf{P}^{1}$. Let $y \in \mathbf{P}^{1}$ such that $\overline{B Y}=\mathbf{P}^{1}$ and hence $T=\{g \in G \mid g y=y\}$ is isomorphic to $\mathbf{G}_{m}$. The operation of $\mathbf{G}_{m} \simeq T$ on the fibre $\phi^{-1}(y) \simeq \mathbf{P}^{1} \times \mathbf{P}^{1}$ is, up to an automorphism of $\mathbf{P}^{1} \times \mathbf{P}^{1}$, given by $(u, v) \rightarrow\left(t^{a} u, t^{b} v\right)$ for $t \in \mathbf{G}_{m},(u, v)$ $\in \mathbf{P}^{1} \times \mathbf{P}^{1}$, where $a, b$ are integers. $(a, b) \neq 0$ since $\mathrm{SL}_{2}$ has an open orbit on $X$ lying over $\mathbf{P}^{1}$. Therefore there exists a $T \simeq \mathbf{G}_{m}$-orbit on $\mathbf{P}^{1} \times \mathbf{P}^{1} \simeq$ $\varphi^{-1}(y)$ whose closure is $\mathbf{P}^{1} \times v_{0}$ or $u_{0} \times \mathbf{P}^{1}$. We may assume that the closure is $\mathbf{P}^{1} \times v_{0}$. Then if for general point $u \in \mathbf{P}^{1}$, we put $D^{\prime}=B\left(u, v_{0}\right)$ 
$\subset X$ and $D=\bar{D}^{\prime}$, then $D$ is a divisor since the stabilizer at $\left(u, v_{0}\right)$ of $B$ is finite. It follows from the construction $D \cap \phi^{-1}(y)=D \cap\left(\mathbf{P}^{1} \times \mathbf{P}^{1}\right)=$ $\mathbf{P}^{1} \times v_{0}$. This is impossible since by Theorem (3.1), [Mo], $\mathbf{P}^{1} \times v_{0}, u_{0} \times \mathbf{P}^{1}$ belong to the same extremal ray but $\left(D . \mathbf{P}^{1} \times a\right)=0,\left(D . b \times \mathbf{P}^{1}\right)=1$.

Lemma (10.7). If $(G, X) \in \mathscr{C}(\mathrm{J} 11 ; m, \ell)$, then $\rho(X) \geq 3(m \geq 2, \ell \geq 2$ or $m=1, \ell \geq 3)$.

Proof. By Lemma (10.4), Lemma (10.5), Lemma (10.6) and [Mo], it is sufficient to prove that $\rho(X) \neq 1$ and this is done by the same argument as in the Proof of Lemma (8.19).

Lemma (10.8). If $(G, X) \in \mathscr{C}(\mathrm{J} 11 ; m, \ell)$ and $\rho(X)=3$, then $X$ is a $\mathbf{P}^{1}$ bundle over $\mathrm{F}_{m}$ and $\mathrm{F}_{m}$-isomorphic to $\mathrm{E}_{m}^{\ell}(k)$ for a suitable integer $k \geq \ell$.

Proof. It follows from Lemma (10.4), Lemma (10.5), Lemma (10.6) and [Mo] that $X$ is a $\mathbf{P}^{1}$-bundle over $\mathrm{F}_{m}$ and $\pi: X \rightarrow \mathrm{F}_{m}$ is $G$-equivariant. Since the Brauer of $\mathrm{F}_{m}$ vanish, there exists a vector bundle $\mathscr{E}$ of rank 2 over $\mathrm{F}_{m}$ such that $X \simeq \mathbf{P}(\mathscr{E})$. The restriction $\mathbf{P}(\mathscr{E}) \mid \mathrm{F}_{m}^{\prime}$ contains the $\mathbf{A}^{1}$ bundle $\mathrm{E}_{m}^{\prime \ell}$ and $D^{\prime}=\left(\mathbf{P}(\mathscr{E}) \mid \mathrm{F}_{m}^{\prime}\right)-E_{m}^{\prime \ell}$ is isomorphic to $\mathrm{F}_{m}^{\prime}$, which is a 2dimensional $G$-orbit on $\mathbf{P}(\mathscr{E}) \mid \mathrm{F}_{m}^{\prime}$ hence on $\mathbf{P}(\mathscr{E})$ (cf. Proof of Lemma (8.17) and Theorem (8.20)). The closure $D$ of $D^{\prime}$ in $\mathbf{P}(\mathscr{E})$ is an irreducible divisor. $D$ contains no fibre of $\mathbf{P}(\mathscr{E})$ over $\mathrm{F}_{m}$. In fact $D$ contains no fibre of $\mathbf{P}(\mathscr{E})$ lying over $\mathrm{F}_{m}^{\prime}$ and if it contained a fibre of $\mathbf{P}(\mathscr{E})$ lying over $\mathrm{F}_{m}-\mathrm{F}_{m}^{\prime}$, since $\mathrm{F}_{m}-\mathrm{F}_{m}^{\prime}=\mathbf{P}^{1}$ is a $G$-orbit, $D$ would contain the surface $\pi^{-1}\left(\mathrm{~F}_{m}-\mathrm{F}_{m}^{\prime}\right)$, which contradicts the irreducibility of $D$. Thus $D$ intersects properly with any fibre of $\pi$. The intersection number $(D . f)=1$ for any fibre lying over $\mathrm{F}_{m}^{\prime}$ hence over $\mathrm{F}_{m}$. This shows that $D$ is non-singular. Since $D$ is an equivariant completion of $D^{\prime} \simeq \mathrm{F}_{m}^{\prime}, D$ is isomorphic to $\mathrm{F}_{m}$ and $\pi \mid D: D \rightarrow$ $\mathrm{F}_{m}$ is an isomorphism. Therefore we get an exact sequence:

$$
0 \longrightarrow L \longrightarrow \mathscr{E} \longrightarrow M \longrightarrow 0,
$$

where $L$ and $M$ are line bundles over $\mathrm{F}_{m}$. Thensoring $L^{-1}$ we may assume $L=\mathcal{O}$ :

$$
0 \longrightarrow \mathcal{O} \longrightarrow \mathscr{E} \longrightarrow M \longrightarrow 0
$$

The restriction of this extension over $\mathrm{F}_{m}^{\prime}$ should be

$$
0 \longrightarrow \mathcal{O}_{\mathrm{F}_{m}^{\prime}} \longrightarrow \mathscr{E} \mid \mathrm{F}_{m}^{\prime} \longrightarrow \mathcal{O}_{\mathrm{F}_{m}^{\prime}}(2-\ell m) \longrightarrow 0 \text {. }
$$

Thus $M \simeq \mathcal{O}_{\mathrm{F}_{m}}(2-\ell m) \otimes \mathcal{O}_{\mathrm{F}_{m}}\left(-k C_{\infty}\right)$, where $C_{\infty}=\mathrm{F}_{m}-\mathrm{F}_{m}^{\prime}$. It follows from 
the argument at the beginning of this section that $(\ell-k) m-2 \leq 0$. The extension (*) is parametrized by

$$
\begin{aligned}
H^{1}\left(\mathrm{~F}_{m}, M^{-1}\right) & =H^{1}\left(\mathrm{~F}_{m}, \mathcal{O}_{\mathrm{F}_{m}}(l m-2) \otimes \mathcal{O}_{\mathrm{F}_{m}}\left(-k C_{\infty}\right)\right) \\
& \simeq H^{1}\left(\mathbf{P}^{1}, \mathcal{O}_{\mathbf{P}_{1}}(l m-2) \otimes S^{k}\left(\mathcal{O}_{\mathbf{P}_{1}} \oplus \mathcal{O}_{\mathbf{P}_{1}}(-m)\right)\right. \\
& \simeq \bigoplus_{j=0}^{k} H^{1}\left(\mathbf{P}^{1}, \mathcal{O}_{\mathbf{P}_{1}}(l m-2-j m)\right)
\end{aligned}
$$

and the extension $(* *)$ by

$$
H^{1}\left(\mathrm{~F}_{m}^{\prime}, \mathcal{O}_{\mathrm{F}_{m}^{\prime}}(\ell m-2)\right) \simeq \underset{j \geq 0}{\oplus} H^{1}\left(\mathbf{P}^{1}, \mathcal{O}_{\mathbf{P} 1}(\ell m-2-j m)\right)
$$

by (1.2.1) and (1.3.1). Hence the restriction $H^{1}\left(\mathrm{~F}_{m}, M^{-1}\right) \rightarrow H^{1}\left(\mathrm{~F}_{m}^{\prime}, M^{-1}\right)$ is injective and the extension defining $\mathscr{E}$ is the cone used to define $\mathrm{E}_{m}^{\ell}(k)$.

We have thus proved

Theorem (10.9). Any element of $\mathscr{C}\left(\right.$ Aut $\left.\mathrm{E}_{m}^{\prime \ell}, \mathrm{E}_{m}^{\prime}\right)=\mathscr{C}(\mathrm{J} 11 ; m, \ell)(m \geq 2$, $\ell \geq 2$ or $m=1, \ell \geq 3$ ) can be equivariantly blown-down to a relatively minimal element of the ordered set $\mathscr{C}(\mathrm{J} 11 ; m, \ell)$ and the centers of the blow-down are curves isomorphic to $\mathbf{P}^{1}$. The relatively minimal elements in $\mathscr{C}(\mathrm{J} 11 ; m, \ell)$ are $\left(\operatorname{Aut}^{0} \mathrm{E}_{m}^{\ell}(j), \mathrm{E}_{m}^{\ell}(j)\right)(j \geq \ell)$.

Remark (10.10). As in the section 8, we can show that the $\left(\right.$ Aut $\left.^{0} \mathrm{E}_{m}^{\ell}(j), \mathrm{E}_{m}^{\ell}(j)\right)(j \geq \ell)$ are related each other by equivariant elementary transformations.

\section{§11. Equivariant completions of J12}

Let us recall the definition of the operation (J12). Let $\pi: C_{1} \rightarrow C_{2}$ be an étale 2-covering of a non-singular open rational curve $C_{2}$ by an irreducible curve $C_{1}$ of genus $g \geq 1$ so that $C_{1}$ is an elliptic or hyperelliptic curve of genus $g$. Let $c$ be the involution of $C_{1}$ giving $\pi$ so that $C_{1} \mid\langle c\rangle$ $\simeq C_{2}$ and we denote by $i$ an involution of $C_{1} \times \mathbf{P}^{1} \times \mathbf{P}^{1}$ :

$$
i(t, x, y)=(\iota t, y, x) \quad \text { for } t \in C_{1}, x, y \in \mathbf{P}^{1} .
$$

We let operate $\mathrm{SL}_{2}$ on $C_{1} \times \mathbf{P}^{1} \times \mathbf{P}^{1}: h(t, x, y)=(t, h x, h y)$ for $(t, x, y) \in C_{1}$ $\times \mathbf{P}^{1} \times \mathbf{P}^{1}$, where for $h \in \mathrm{SL}_{2} z \in \mathbf{P}^{1}, h z$ denote the usual action of $\mathrm{SL}_{2}$ on $\mathbf{P}^{1}$. The operation of $\mathrm{SL}_{2}$ on $C_{1} \times\left(\mathbf{P}^{1} \times \mathbf{P}^{1}\right.$ - diagonal $)$ commutes with the involution $i$ and thus defines an operation $\left(\mathrm{SL}_{2}, X_{\pi}\right)$, where $X_{\pi}=\left(\mathbf{P}^{1} \times \mathbf{P}^{1}\right.$. diagonal) $/\langle i\rangle$. See (3.9), [U4].

Let us construdt a model. Let $g$ be a non-negative integer and $n=g+1$. Let $V$ be an irreducible $\mathrm{SL}_{2}$-module of dimension 3 so that 
we identify $V$ with the vector space of the homogeneous polynomials of degree 2 in $x, y$. The operation of $\mathrm{SL}_{2}$ on the vector space $k x \oplus k y$ is in the usual way. By letting $\mathrm{SL}_{2}$ operator on $\mathcal{O}_{\mathbf{P} 1}(n)$ trivially, $\mathrm{SL}_{2}$ operates on a vector bundle $\mathscr{E}=\left(\mathcal{O}_{\mathbf{P} 1} \otimes_{k} V\right) \oplus \mathcal{O}_{\mathbf{P} 1}(n)$ over $\mathbf{P}^{1}$. We use the basis $a$, $b, c$ on $V: g(x, y)=a x^{2}+b x y+c y^{2} \in V$. Denoting by $z$ the fibre coordinate of $\mathcal{O}_{\mathbf{P} 1}(n)$ locally, if $f(t) \in H^{0}\left(\mathbf{P}^{1}, \mathcal{O}(2 u)\right), b^{2}-4 a c+f(t) z^{2}$ is an $\mathrm{SL}_{2}$-invariant section of $H^{0}\left(\mathbf{P}^{1}, S^{2}(\mathscr{E})\right)$. Therefore we get a quadric bundle $p: X_{f}=$ $\left\{b^{2}-4 a c+f(t) z^{2}=0 \mid\left(a x^{2}+b x y+c y^{2}, z\right) \in \mathbf{P}(\mathscr{E})\right\} \rightarrow \mathbf{P}^{1}$ on which $\mathrm{SL}_{2}$-operates. From now on we assume that the homogeneous polynomial $f(t)$ of degree $2 n$ has only simple roots so that $y^{2}=f(t)$ defines a hyperelliptic (or elliptic) curve.

LEMMA (11.1). If $f(t)=0$ has only simple roots, then $X_{f}$ is non-singular and projective.

Proof. In fact, locally $X_{f}$ is defined by $\left\{(t ; a, b, c, z) \in \mathbf{A}^{1} \times \mathbf{P}^{3} \mid b^{2}-\right.$ $\left.4 a c+f(t) z^{2}=0\right\}$. The smoothness of $X_{f}$ follows from this local expression. The projectivity of $X_{f}$ is obvious.

LEMma (11.2). Let $\pi: C \rightarrow \mathbf{P}^{1}$ be the hyperelliptic curve defined by $y^{2}=f(t)$. Then $\left(\mathrm{SL}_{2}, X_{\pi}\right)$ and $\left(\mathrm{SL}_{2}, X_{\pi}\right)$ are mutually isomorphic as law chunks of algebraic operation.

Proof. If we add $y=\sqrt{f(t)}$, then over $k(t, y)$ the quadratic form $b^{2}-4 a c+f(t) z^{2}$ is isomorphic to; $b^{2}-4 a c-w^{2}$. Namely $X_{f} \otimes_{\mathbf{p}_{1}} C$ is birationally equivalent to the product $C \times$ (quadric surface). Now the descent datum on $C \times$ (quadric surface) defining $X_{f}$ is $(a, b, c, w) \rightarrow(a, b$, $c,-w)$ if written in terms of the coordinate system on $C \times \mathbf{P}^{3}$. Let us see how the quadric is identified with $\mathbf{P}^{1} \times \mathbf{P}^{1}$ embedded in $\mathbf{P}^{3}$ by $\mathbf{P}^{1} \times \mathbf{P}^{1} \ni$ $\left(\left(x_{0}, x_{1}\right) ;\left(u_{0}, u_{1}\right)\right) \rightarrow\left(x_{0} u_{0}, x_{0} u_{1}, x_{1} u_{0}, x_{1} u_{1}\right) \in \mathbf{P}^{3}$. If $\mathrm{SL}_{2}$ operates diagonally on $\mathbf{P}^{1} \times \mathbf{P}^{1}, \mathrm{SL}_{2}$-module $H^{\circ}\left(\mathbf{P}^{1} \times \mathbf{P}^{1}, \mathcal{O}(1,1)\right)$ is decomposed into the direct sum $U_{3} \oplus U_{1}$, where $U_{3}$ is the irreducible $\mathrm{SL}_{2}$-module of degree $i$. The explicit decomposition is $H^{0}\left(\mathbf{P}^{1} \times \mathbf{P}^{1}, \mathcal{O}(1,1)\right) \simeq H^{0}\left(\mathbf{P}^{1}, \mathcal{O}(1)\right) \otimes H^{0}\left(\mathbf{P}^{1},(1)\right)=\left(k x_{0} u_{0}+\right.$ $\left.k\left(x_{0} u_{1}+x_{1} u_{0}\right)+k x_{1} u_{1}\right) \oplus k\left(x_{0} u_{1}-x_{1} u_{0}\right)$. Thus to connect $X_{f}$ with $X_{\pi}$ we had better consider the embedding $\mathbf{P}^{1} \times \mathbf{P}^{1} \rightarrow \mathbf{P}^{3}$ given by $\left(\left(x_{0}, x_{1}\right) ;\left(u_{0}, u_{1}\right)\right)$ $\rightarrow\left(x_{0} u_{0}, x_{0} u_{1}+x_{1} u_{0}, x_{1} u_{1}, x_{0} u_{1}-x_{1} u_{0}\right)$ so that the defining equation of the image is $X_{1}^{2}-4 X_{0} X_{2}-X_{3}^{2}=0$. Therefore the involution $(a, b, c, w)(a, b$, $c,-w)$ on the quadric just corresponds to the automorphism of $\mathbf{P}^{1} \times \mathbf{P}^{1}$ interchanging the factors. Now Lemma follows from the definition of $X_{\pi}$. 
Remark (11.3). We may expect to argue as in the preceding cases trying to exclude the cases (3.3.3), (3.3.4) and (3.3.5) in [Mo]. But the case (3.3.4) does occur as we see below (see (11.18)). Therefore we examine equivariant completions studying closely the operation $\left(\mathrm{SL}_{2}, X_{f}\right)$ in Lemma (11.1) and its equivariant blow-ups.

LEMMA (11.4). The $\mathrm{SL}_{2}$-invariant reduced irreducible effective divisors on $X_{f}$ are; (1) the fibre $p^{-1}(t), t \in \mathbf{P}^{1}$ which is isomorphic to $\left(\mathrm{SL}_{2}, \mathbf{P}^{1} \times \mathbf{P}^{1}\right)$ with diagonal $\mathrm{SL}_{2}$-action if $f(t) \neq 0$ and to the singular quadric $\left\{u^{2}+v^{2}\right.$ $\left.+w^{2}=0\right\} \subset \mathbf{P}^{3}$ if $f(t)=0$, (2) $D_{\infty}=\left\{b^{2}-4 a b=0 \mid\left(a x^{2}+b x y+c y^{2}, 0\right) \epsilon\right.$ $\left.X_{f} \subset \mathbf{P}(\mathscr{E})\right\}$ which is isomorphic to $\left(\mathrm{SL}_{2}, \mathbf{P}^{1} \times \mathbf{P}^{1}\right)$ with $\mathrm{SL}_{2}$-action through the first factor.

Remark (11.5). Since $\mathrm{SL}_{2}$ is a semi-simple part of $\mathrm{Aut}^{0} Z \simeq \mathrm{Aut}^{0} \mathrm{~F}_{2}$ by [U3], the operation of $\mathrm{SL}_{2}$ on the singular quadric $Z$ is essentially unique and given as follows: Let $U_{3}$ denote the irreducible $\mathrm{SL}_{2}$-module of dimension 3 which we identify with the vector space of the homogeneous polynomials of degree 2 in 2 variables $x, y$. Then $U_{3}$ has an $\mathrm{SL}_{2}$-invariant, the discriminant $D$ of the degree 2 polynomial, $D\left(a x^{2}+b x y+c y^{2}\right)=b^{2}$ - 4ac. $k$ being the trivial $\mathrm{SL}_{2}$-module, $\mathrm{SL}_{2}$ acts on the direct sum $U_{3} \oplus k$ hence on $\mathbf{P}^{3}=\mathbf{P}\left(U_{3} \oplus k\right)$ leaving the singular quadric $b^{2}-4 a v=0$ in $\mathbf{P}^{3}$ invariant.

Proof. Lemma follows from the construction of $X_{f}$.

general fibre $\simeq \mathbf{P}^{1} \times \mathbf{F}^{1}$, singular fibre $\simeq$ the singular quadric in $\mathbf{P}^{3}$

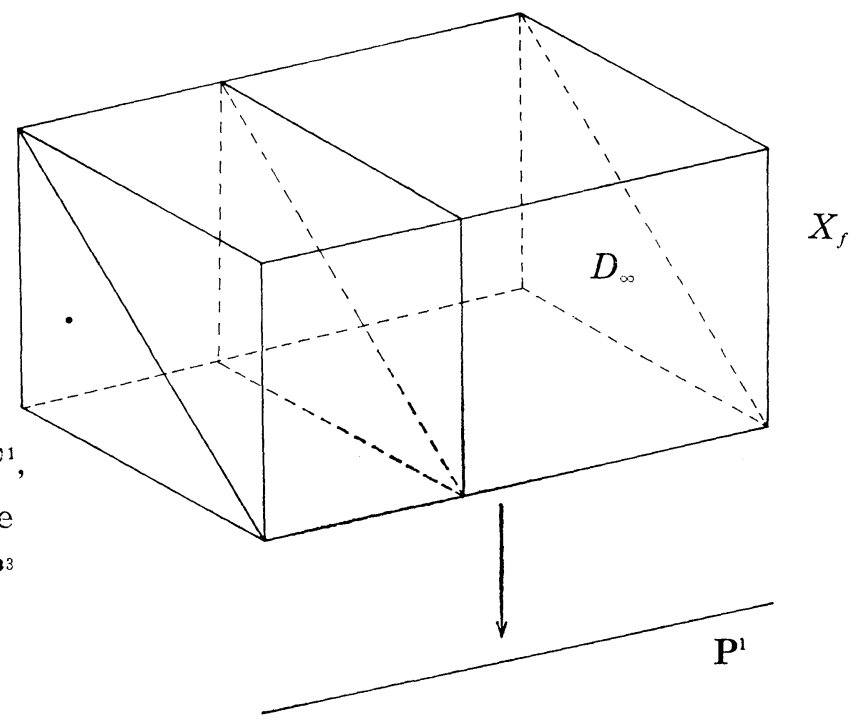


Lemma (11.6). The $\mathrm{SL}_{2}$-fixed points on $X_{f}$ are the singular points of the singular fibres. If we blow up $X_{f}$ at one of the $\mathrm{SL}_{2}$-fixed points, $\mathrm{SL}_{2}$ operates on the exceptional divisor $\mathbf{P}^{2}$ through irreducible $\mathrm{SL}_{2}$-module of dimension 3.

Proof. The first assertion of the Lemma is a consequence of the construction of $X_{f} . \quad X_{f}$ is locally defined by $\left\{(t ;(a . b, c, z)) \in \mathbf{A}^{1} \times \mathbf{P}^{3} \mid b^{2}-4 a c\right.$ $\left.+f(t) z^{2}=0\right\}$. We may assume that the fixed point is in the fibre $p^{-1}(0)$, namely $t=0$ is a simple zero of $f(t)=0$. Hence locally for the usual topology at the singular point $y$ of the singular fibre $p^{-1}(0)$, we may assume that $X_{f}$ is defined by $\left\{(t, a, b, c) \in \mathbf{A}^{4} \mid b^{2}-4 a c+t=0\right\}$ and therefore $X_{f}$ is isomorphic to, around $(0 ;(0,0,0)), \mathbf{A}^{3}$ with irreducible $\mathrm{SL}_{2}$-action on $\mathbf{A}^{3}$.

Notation (11.7). We denote by $W_{f}$ the blow-up of $X_{f}$ at all the $\mathrm{SL}_{2}$-fixed points of $X_{f}$.

LEMma (11.8). The $\mathrm{SL}_{2}$-invariant irreducible reduced effective divisors on $W_{f}$ are; (1) the proper transforms of $\mathrm{SL}_{2}$-invariant divisors on $X_{f}$, (2) the exceptional divisors isomorphic to $\mathbf{P}^{2}$. The proper transforms of the singular fibres are isomorphic to $\mathrm{F}_{2}$. There is no $\mathrm{SL}_{2}$-fixed point on $W_{f}$.

Proof. Since $\mathrm{SL}_{2}$ operates on the exceptional divisor $\mathbf{P}^{2}$ through the irreducible representation, $\mathrm{SL}_{2}$-orbit decomposition of $\mathbf{P}^{2}$ is $\mathrm{SL}_{2} / \mathbf{G}_{m}$, which is an open orbit, and $\mathrm{SL}_{2} / B \simeq \mathbf{P}^{1}$, which is a conic in $\mathbf{P}^{2}$.

Here we give a table of intersections of 2 divisors on $W_{f}$. We denote by $q: W_{f} \rightarrow X_{f}$ the blow-up and by $q^{-1}[D]$ the proper transform of $D$.

The statement at $(A, B)$ in the table

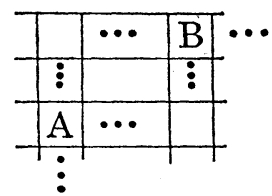

describes the curve

$A \cap B$ as a curve on $A$.

Table (11.9)

\begin{tabular}{|c|c|c|c|c|}
\hline & $\begin{array}{l}q^{-1}\left(\bar{D}_{\infty}\right) \simeq \\
\mathbf{P}^{1} \times \mathbf{P}^{1}\end{array}$ & $\begin{array}{c}q^{-1}(\text { smooth fibre }) \\
\simeq \mathbf{P}^{1} \times \mathbf{P}^{1}\end{array}$ & $q^{-1}[$ singular tibre] & $\begin{array}{l}\text { exceptional } \\
\text { divisor } \simeq \mathbf{P}^{2}\end{array}$ \\
\hline$q^{-1}\left(D_{\infty}\right) \simeq \mathbf{P}^{1} \times \mathbf{P}^{1}$ & & $\mathbf{P}^{1} \times a$ in $\mathbf{P}^{1} \times \mathbf{P}^{1}$ & $\mathbf{P}^{1} \times a$ in $\mathbf{P}^{1} \times \mathbf{P}^{1}$ & empty \\
\hline $\begin{array}{c}q^{-1}(\text { smooth fibre }) \\
\simeq \mathbf{P}^{1} \times \mathbf{P}^{1}\end{array}$ & $\begin{array}{l}\text { diagonal } \\
\mathbf{P}^{1} \subset \mathbf{P}^{1} \times \mathbf{P}^{1}\end{array}$ & & empty & empty \\
\hline$q^{-1}\left[\underset{\simeq \mathrm{F}_{2}}{\operatorname{singuar}}\right.$ fibre] & $\begin{array}{l}\text { 0-section } \\
\text { in } \mathrm{F}_{2}\end{array}$ & empty & & $\begin{array}{l}\infty \text {-section } \\
\text { in } F_{2}\end{array}$ \\
\hline $\begin{array}{l}\text { exceptional } \\
\text { divisor } \simeq \mathrm{P}^{2}\end{array}$ & empty & empty & $\begin{array}{l}\text { non-singular } \\
\text { conic in } \mathbf{P}^{2}\end{array}$ & \\
\hline
\end{tabular}


where the $\infty$-section of $F_{2}$ is unique section of the ruled surface $F_{2}$ with self-intersection number -2 and a 0 -section is a section of the ruled surface disjoint from the $\infty$-section, whose self-intersection number is necessarily equal to 2 .

Corollary (11.10). Let $C$ be an irreducible reduced $\mathrm{SL}_{2}$-invariant curve on $W_{f}$. (a) $C$ is an $\mathrm{SL}_{2}$-orbit hence isomorphic to $\mathbf{P}^{1}$.

(b) $C$ is contained in a fibre $q^{-1} \circ p^{-1}(t)$ for a some point $t \in \mathbf{P}^{1}$.

(c) $C$ is an intersection of 2 irreducible reduced effective divisors.

(d) If 2 irreducible reduced effective divisors on $W_{f}$ intersect, they intersect transversely along $\mathbf{P}^{1}$.

(e) Any 3 distinct irreducible reduced effective divisors have no point in common.

Proof. This is a consequence of Lemma (11.8) and the construction of $X_{f}$.

Now we describe the $\mathrm{SL}_{2}$-invariant divisors on an equivariant blow-up of $W_{f}$.

Lemma (11.11). Let $f_{2}: \tilde{Y} \rightarrow W_{f}$ be a sequence of $\mathrm{SL}_{2}$-equivariant blowups: $\chi_{i, i+1}: X_{i} \rightarrow X_{i+1}(0 \leq i \leq m+1)$ is an $\mathrm{SL}_{2}$-equivariant blow-up at an irreducible center, $X_{0}=\tilde{Y}, X_{m}=X_{f}$ and $f_{2}=\chi_{m-1 m} \circ \chi_{m-2 m-1} \circ \chi_{01}$. For $1 \leq i$ $\leq m$, we have the following.

(a) There is no $\mathrm{SL}_{2}$-fixed point on $X_{i}$.

(b) Any $\mathrm{SL}_{2}$-invariant irreducible reduced effective divisor on $X_{i}$ is smooth.

(c) Any $\mathrm{SL}_{2}$-invariant (integral) curve $C$ on $X_{i}$ has the following properties:

(c1) $C$ is an $\mathrm{SL}_{2}$-orbit hence isomorphic to $\mathbf{P}^{1}$,

(c2) $C$ is contained in a fibre $\chi_{i m}^{-1} \circ p^{-1}(t)$ for a some point $t \in \mathbf{P}^{1}$,

(c3) $C$ is an intersection of $2 \mathrm{SL}_{2}$-invariant irreducible reduced effective divisors $A, B$ such that $C=A \cap B,\left(C^{2}\right)_{A} \neq 0$.

(d) If 2 irreducible reduced effective divisors on $X_{i}$ intersect, they intersect transversely along $\mathbf{P}^{1}$.

(e) Any 3 distinct irreducible reduced effective divisors on $X_{i}$ have no point in common.

(f) The exceptional divisor $E_{i} \subset X_{i}(0 \leq i \leq m-1)$ for $X_{i, i+1}$ is isomorphic to the ruled surface $\mathrm{F}_{\ell_{i}}$ for suitable integer $\ell_{i} \geq 1$ with non-trivial $\mathrm{SL}_{2}$-action. 
Proof. (a) follows from Lemma (11.8) since the image of a fixed point is $\mathrm{SL}_{2}$-fixed. We prove Lemma (11.11) by descending induction on $i$. If $i=m$, Lemma (11.11) is equivalent to Corollary (11.10). Now we have to prove the assertions for $i$ assuming the lemma for $i+1$. Since there is no fixed point on $X_{i+1}, \chi_{i, i+1}: X_{i} \rightarrow X_{i+1}$ is the blow-up of $X_{i+1}$ at an irreducible curve hence by (b) at $\mathbf{P}^{1}$. Therefore the exceptional divisor $E_{i}$ is a ruled surface $\mathrm{F}_{\ell_{i}}$. Since if $\mathrm{SL}_{2}$ operates on $\mathrm{F}_{\ell}$ non-trivially and if $\ell \geq 1, \mathrm{SL}_{2}$-orbit decomposition of $\mathrm{F}_{\ell}$ is $\mathrm{F}_{\ell}=$ open orbit (0-section) $U$ ( $\infty$-section), by Lemma (1.11) and (1.3.3) the other assertions follow once we prove (c3) for $\mathrm{SL}_{2}$-invariant curves on $E_{i} \subset X_{i}$.

To illustrate our argument, let us first prove the lemma for $i=m$ -1 , namely $\chi_{m-1 m}: X_{m-1} \rightarrow X_{m}=W_{f}$ is a blow-up at an irreducible nonsingular $\mathrm{SL}_{2}$-invariant curve. We give a formal induction later. We verify $\ell_{m-1} \geq 1$ case by case. Let $h: Y \rightarrow W_{f}$ be the blow-up of $W_{f}$ at the intersection $q^{-1}\left(D_{\infty}\right) \cap q^{-1}$ (smooth fibre) (see Fig. (11.11.1)) and $E$ be the exceptional divisor for $h$.

Fig. (11.11.1)

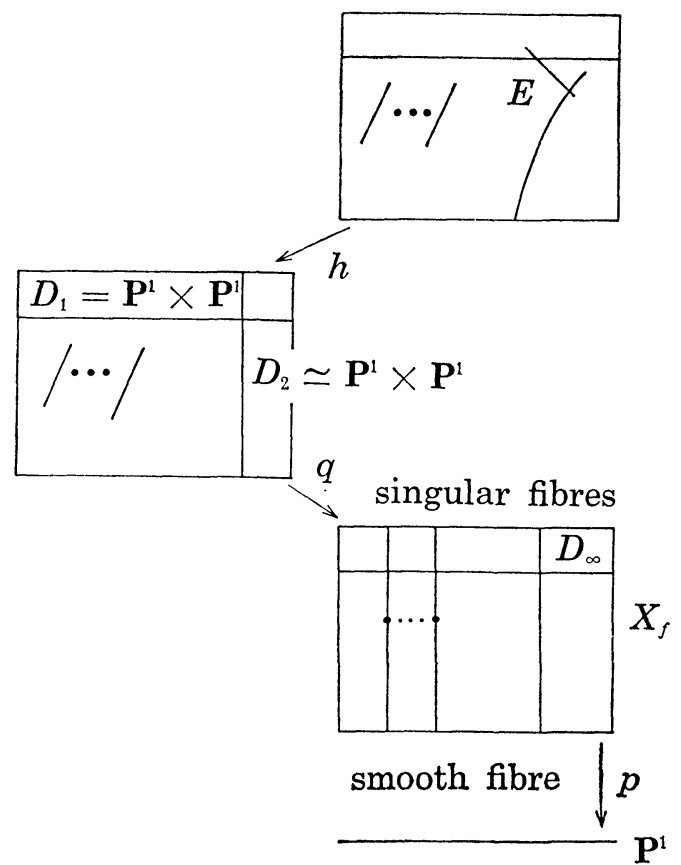

Since $D_{1}=q^{-1}\left(D_{\infty}\right)$ and $D_{2}=q^{-1}$ (smooth fibre) intersect transversely along the curve isomorphic to $\mathbf{P}^{1}, E$ is isomorphic to $\mathrm{F}_{\ell}$ for some integer $\ell \geq 0$. 
Moreover $h^{-1}\left[D_{1}\right] \cap E$ and $h^{-1}\left[D_{2}\right] \cap E$ are disjoint sections of ruled surface $\mathrm{F}_{\ell}$ since $D_{1}$ and $D_{2}$ intersect transversely along the center of the blow-up. We calculate the intersection numbers $\left(h^{-1}\left[D_{1}\right]^{2} . E\right)$ and $\left(h^{-1}\left[D_{2}\right]^{2} . E\right)$. Since $D_{2}$ is a fibre of $p \circ q, h^{*} D_{2} . E=0$ hence

$$
\begin{aligned}
0 & =\left(h^{-1}\left[D_{2}\right] \cdot h^{*} D_{2} \cdot E\right)=\left(h^{-1}\left[D_{2}\right] \cdot h^{-1}\left[D_{2}\right]+E \cdot E\right) \\
& =\left(h^{-1}\left[D_{2}\right] \cdot h^{-1}\left[D_{2}\right] \cdot E\right)+\left(h^{-1}\left[D_{2}\right] \cdot E \cdot E\right)=\left(h^{-1}\left[D_{2}\right] \cdot h^{-1}\left[D_{2}\right] \cdot E\right)+2
\end{aligned}
$$

by the table (11.9). Therefore

$$
\left(h^{-1}\left[D_{2}\right] \cdot h^{-1}\left[D_{2}\right] \cdot E\right)=-2 \text { and }\left(h^{-1}\left[D_{1}\right], h^{-1}\left[D_{1}\right] . E\right)=2
$$

since $h^{-1}\left[D_{2}\right] \cap E$ is a section of $\mathrm{F}_{6}$ disjoint from $h^{-1}\left[D_{2}\right] \cap E$. We have thus proved $\ell=2$ and $E$ is isomorphic to $\mathrm{F}_{2}$ :

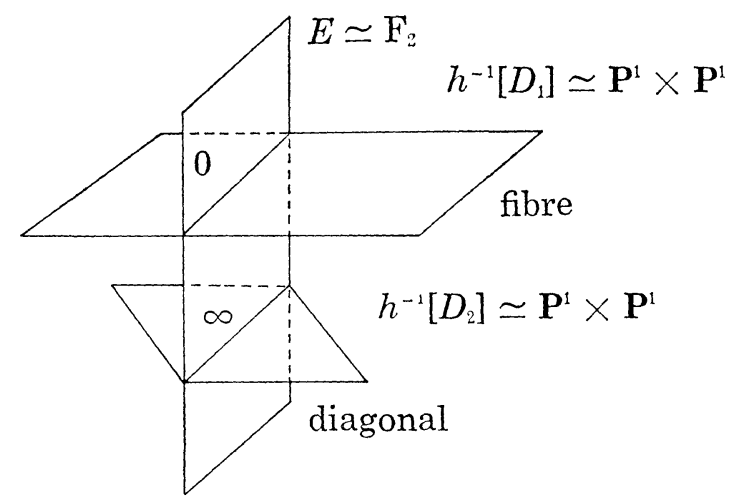

Let now $j: Z \rightarrow W_{f}$ be the blow up of $W_{f}$ at the intersection of an exceptional divisor $D_{3}$ for $q$ and $q^{-1}$ [a singular fibre passing through $q\left(D_{3}\right)$ ] which we denote by $D_{4}$ (see Fig. (11.11.2)).

For the same reason as in case of $h$, the exceptional divisor $J$ for $j$ is isomorphic to $F_{\ell}$ for some integer $\ell \geq 0$ and

$$
\begin{aligned}
0 & =\left(j^{-1}\left[D_{3}\right] .(\text { fibre of } p \circ q \circ j) \cdot J\right)=\left(j^{-1}\left[D_{3}\right] \cdot j^{*}\left(2 D_{3}+D_{4}\right) \cdot J\right) \\
& =\left(j^{-1}\left[D_{3}\right] \cdot j^{-1}\left[2 D_{3}\right]+j^{-1}\left[D_{4}\right]+3 J \cdot J\right) \\
& =\left(j^{-1}\left[D_{3}\right] \cdot j^{-1}\left[2 D_{3}\right] \cdot J\right)+\left(j^{-1}\left[D_{3}\right] \cdot j^{-1}\left[D_{4}\right] \cdot J\right)+\left(j^{-1}\left[D_{3}\right] \cdot 3 J . J\right) \\
& =2\left(j^{-1}\left[D_{3}\right] \cdot j^{-1}\left[D_{3}\right] . J\right)+12
\end{aligned}
$$

by Table (11.9). Hence $\left(j^{-1}\left[D_{3}\right] \cdot j^{-1}\left[D_{3}\right] . J\right)=-6 . j^{-1}\left[D_{4}\right] \cap J \subset J$ is a section disjoint from the section $j^{-1}\left[D_{3}\right] \cap J \subset J$ hence $\left(j^{-1}\left[D_{4}\right] \cdot j^{-1}\left[D_{4}\right] . J\right)=6$. Let us check this by another calculation. For the same reason as in preceding cases, 
Fig. (11.11.2)

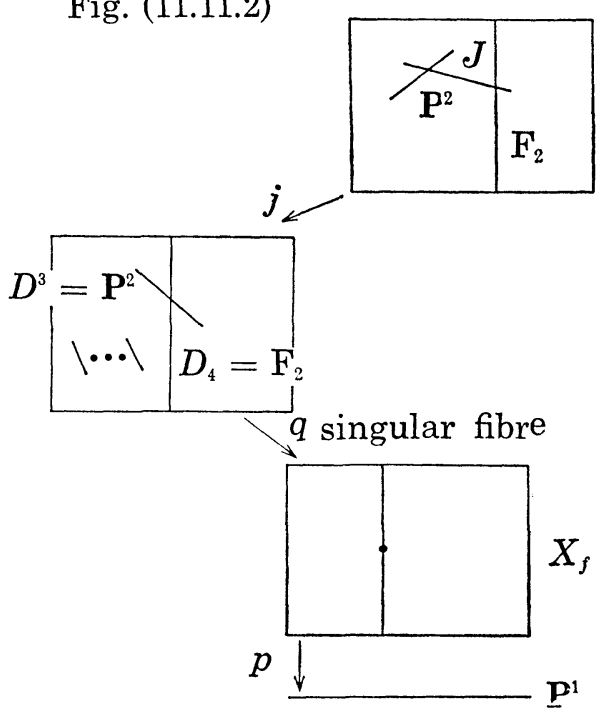

$$
\begin{aligned}
0 & =\left(j^{-1}\left[D_{4}\right] . \text { fibre of } p \circ q \circ j . J\right)=\left(j^{-1}\left[D_{4}\right] \cdot j^{-1}\left[2 D_{3}\right]+j^{-1}\left[D_{4}\right]+3 J . J\right) \\
& =2\left(j^{-1}\left[D_{4}\right], j^{-1}\left[D_{3}\right] . J\right)+\left(j^{-1}\left[D_{4}\right] \cdot j^{-1}\left[D_{4}\right] . J\right)+3\left(j^{-1}\left[D_{4}\right] . J . J\right) \\
& =\left(j^{-1}\left[D_{4}\right] \cdot j^{-1}\left[D_{4}\right] . J\right)-6 .
\end{aligned}
$$

Let $r: V \rightarrow W_{f}$ be the blow-up of $W_{f}$ at the intersection of $D_{5}=q^{-1}\left(D_{\infty}\right)$ and $D_{6}=q^{-1}$ [singular fibre](see Fig. (11.11.3)). We denote by $D$ the irreducible exceptional divisor for $q$ intersecting with $D_{6}$.

Fig. (11.11.3)

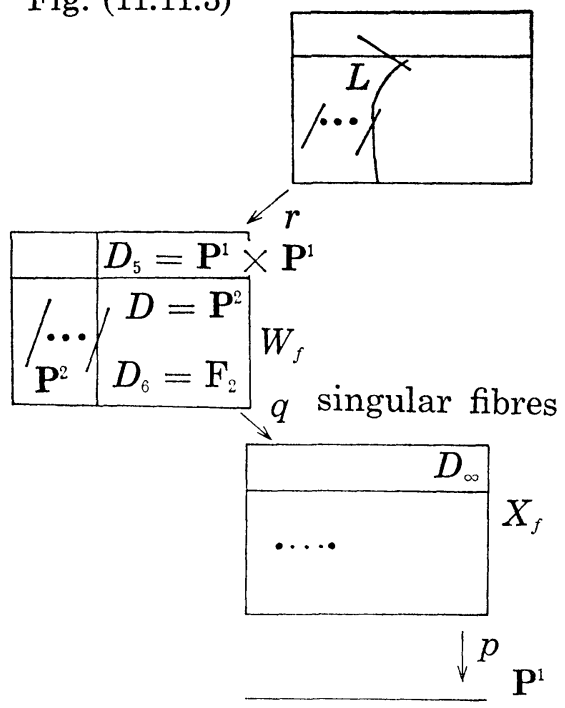


We denote by $L$ the exceptional divisor for $r$. For the same reason as before $0=\left(r^{-1}\left[D_{6}\right]\right.$. (fibre of $\left.\left.p \circ q \circ r\right) . L\right)=\left(r^{-1}\left[D_{6}\right] \cdot r^{-1}\left[D_{6}\right]+L+2 r^{*} D . L\right)$ $=\left(r^{-1}\left[D_{6}\right] \cdot r^{-1}\left[D_{6}\right] . L\right)+\left(r^{-1}\left[D_{6}\right] . L . L\right)=\left(r^{-1}\left[D_{6}\right] \cdot r^{-1}\left[D_{6}\right] . L\right)+2$. Hence $L$ is isomorphic to $\mathrm{F}_{2}$.

Let us now give a rigorous induction. As we have seen at the beginning of the proof, $\chi_{i-1, i}: X_{i-1} \rightarrow X_{i}$ is the blow-up at an $\mathrm{SL}_{2}$-invariant curve $C_{i}$ on $X_{i}$ and it is sufficient to prove (c3) for $\mathrm{SL}_{2}$-invariant curves on $E_{i-1} \subset X_{i-1}$ under the assumption that Lemma (11.11) holds for $X_{i}$. For the center $C_{i}$, we can find a smooth $\mathrm{SL}_{2}$-invariant divisor $A_{i}$ such that $C_{i} \subset A, p \circ q \circ \chi_{i m}(A)$ is a point of $\mathbf{P}^{1}$ and such that $\left(C_{i}^{2}\right)_{A_{i}} \neq 0$. Let $B_{i}$ be the $G$-invariant smooth irreducible divisor on $X_{i}$ with $C_{i}=A_{i} \cap B_{i}: B_{i}$ exists by (c3) for $X_{i}$.

Fig. (11.11.4)

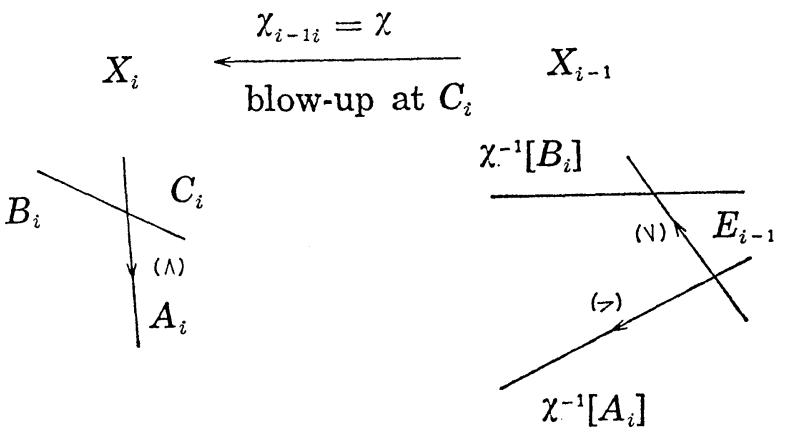

$\chi=\chi_{i-1, i}: X_{i-1} \rightarrow X_{i}$ is the blow-up at $C_{i}=A_{i} \cap B_{i}$ as Fig. (11.11.4) shows. We have:

$$
\begin{aligned}
0= & \left(\left(a \text { fibre of } p \circ q \circ \chi_{i m}\right) \cdot E_{i-1} \cdot \chi^{-1}\left[A_{i}\right]\right)_{X_{i-1}} \\
= & \left(\left(a \chi^{-1}\left[A_{i}\right]+b E_{i-1}+\text { other divisors disjoint from } E_{i-1}\right) E_{i-1} \cdot \chi^{-1}\left[A_{i}\right]\right)_{X_{i-1}} \\
& \quad \text { (here } a, b \text { are positive integers }) \\
= & a\left(\chi^{-1}\left[A_{i}\right] . E_{i-1} \cdot \chi^{-1}\left[A_{i}\right]\right)_{X_{i-1}}+b\left(E_{i-1} \cdot E_{i-1} \cdot \chi^{-1}\left[A_{i}\right]\right)_{X_{i-1}} .
\end{aligned}
$$

Thus $\left(E_{i-1} \cdot E_{i-1} \cdot \chi^{-1}\left[A_{i}\right]\right)_{X_{i-1}} \neq 0$ and $E_{i-1} \simeq \mathrm{F}_{\ell_{i-1}}$ with $\ell_{i} \geq 1$ since $E_{i-1} \cap$ $\chi^{-1}\left[A_{i}\right]$ and $E_{i-1} \cap \chi^{-1}\left[B_{i}\right] \subset E_{i-1}$ are disjoint sections (cf. (1.3.3)). This proves (c3) for $i-1$. For, since there is no $\mathrm{SL}_{2}$-fixed point on $X_{i-1}$, new born $\mathrm{SL}_{2}$-invariant curves on $X_{i-1}$ are $\chi^{-1}\left[B_{i}\right] \cap E_{i-1}$ and $\chi^{-1}\left[A_{i}\right] \cap E_{i-1}$.

The fibration $p: X_{f} \rightarrow \mathrm{P}^{1}$ is a dell Pezzo fibration of Theorem (3.5) (3.5.2), [Mo]. In fact we show (see Example (11.18)).

Lemma (11.12). Pic $X_{f}$ is generated by $D_{\infty}$ and $p^{*}$ Pic $\mathbf{P}^{1}$. In partic- 
ular the Picard number $\rho\left(X_{f}\right)=2$.

Proof. As in the Proof of Lemma (11.2), let $C \rightarrow \mathbf{P}^{1}$ be the hyperelliptic curve defined by the extension $k(\sqrt{f}, t) / k(t)$. Let $B \subset C$ be the set of branch points and $R \subset \mathbf{P}^{1}$ be the set of ramification poits so that $\pi: C-B \rightarrow \mathrm{P}^{1}-R$ is étale. Let us denote $C-B$ by $C_{1}$ and $\mathbf{P}^{1}-R$ by $C_{2}$. As we have seen in the Proof of Lemma (11.2). The restriction $C_{2} \times{ }_{\mathbf{P} 1} X_{f}$ is the quotient of $C_{1} \times \mathbf{P}^{1} \times \mathbf{P}^{1}$ by the involution $i: i(t, x, y)$ $=(\iota t, y, x)$ for $t \in C_{1}, x, y \in \mathbf{P}^{1}$ where $\iota$ is the involution arising from $\pi$. $\operatorname{Pic}\left(C_{1} \times \mathbf{P}^{1} \times \mathbf{P}^{1}\right)$ is isomorphic to Pic $C_{1} \oplus \operatorname{Pic} \mathbf{P}^{1} \otimes \operatorname{Pic} \mathbf{P}^{1}$ hence to Pic $C_{1}$ $\oplus \mathbf{Z}^{\oplus 2}$. By the descent theory,

$$
\text { Pic } C_{2} \times_{\mathbf{P} 1} X_{f} \simeq\left(\operatorname{Pic} C_{1} \oplus \mathbf{Z}^{\oplus 2}\right)^{i} \simeq \mathbf{Z} \text {. }
$$

Given a line bundle $L$ over $X_{f}$. It follows from (11.12.1) that we can find an integer $n$ such that $L\left(n D_{\infty}\right)$ is trivial when restricted over $C_{2} \times_{\mathbf{P} 1} X_{f}$. Therefore $L\left(n D_{\infty}\right) \simeq \mathcal{O}_{X_{f}}(D)$ for some divisor $D$ whose support lies in the singular fibres of $p: X_{f} \rightarrow \mathbf{P}^{1}$. We have thus proved $\operatorname{Pic} X_{f}=\mathbf{Z} D_{\infty} \otimes$ $p^{*}$ Pic $\mathbf{P}^{1}$.

Let now $\left(\mathrm{SL}_{2}, Y\right) \in \mathscr{C}(\mathrm{J} 12, \pi)=\mathscr{C}\left(\mathrm{SL}_{2}, X_{\pi}\right)$. We have an equivariant birational map $f: Y \cdots W_{f}$ by Lemma (11.1). By blowing up $Y$, we can eliminate the indeterminacy of $f$ :

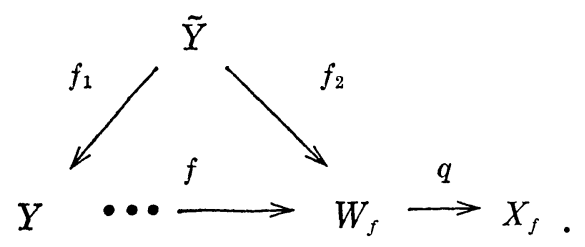

After Hironaka, everything is done $\mathrm{SL}_{2}$-equivariantly. Since there is no $\mathrm{SL}_{2}$-fixed point by Lemma (11.8), the dimension of any fibre of $f_{2} \leq 1$ and hence $f_{2}$ is also a blow-down by Theorem (1.7). We may take $f_{2}$ minimal so that there is no non-trivial factorization;

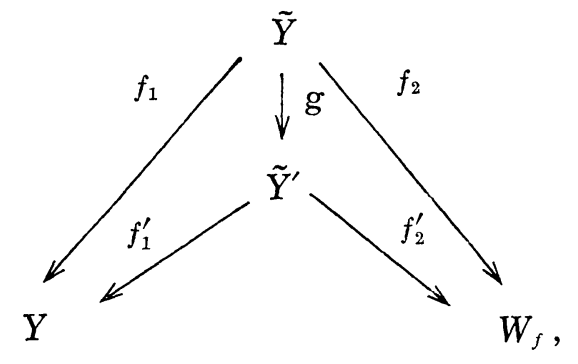


where $g, f_{1}^{\prime}$ and $f_{2}^{\prime}$ are blow-ups. Let us decompose $f_{2}$ into a product of blow ups: $\chi_{i, i+1}: X_{i} \rightarrow X_{i+1} 0 \leq i \leq m-1, X_{0}=\tilde{Y}, X_{m}=W_{f}, f_{2}=\chi_{m-1, m}$ 。 $\chi_{m-2, m-1} \circ \ldots \circ \chi_{01}$ and $\chi_{i, i+1}$ is an $\mathrm{SL}_{2}$-equivariant blow-up of $X_{i+1}$ at nonsingular irreducible curve.

Similarly, let us decompose $f_{1}$ as a product of blow-ups with irreducible centers: $\psi_{j, j+1}: Y_{j} \rightarrow Y_{j+1}(0 \leq j \leq s-1), Y_{0}=\tilde{Y}, Y_{s}=Y$ and $\psi_{j, j+1}$ is a blow-up with a non-singular irreducible center.

Lemma (11.13). Let $D$ be a smooth fibre $p^{-1}(t), t \in \mathbf{P}^{1}$. The proper transform $\left(q \circ f_{2}\right)^{-1}[D]$ is not an exceptional divisor for $f_{1}$.

Proof. $D_{\infty}$ and $p^{* \mathcal{O}_{\mathbf{P} 1}}(1)$ generate Pic $X_{f}$ by Lemma (11.12). The smooth fibre $D$ is a non-singular quadric in $\mathbf{P}^{3}$ hence isomorphic to $\mathbf{P}^{1} \times \mathbf{P}^{1}$. Let us set $\ell_{1}=\mathbf{P}^{1} \times a$ and $\ell_{2}=a \times \mathbf{P}^{1}$. They are curves on $D$ hence on $X_{f}$. It follows from the construction $\left(\ell_{1} . D_{\infty}\right)=\left(\ell_{2} . D_{\infty}\right)=1, \quad\left(\ell_{1} \cdot p^{*} \mathcal{O}_{\mathbf{P}_{1}}(1)\right)$ $=\left(\ell_{2} \cdot p^{*} \mathcal{O}_{\mathbf{p}_{1}}(1)\right)=0$. Therefore $\ell_{1}$ is numerically equivalent to $\ell_{2}$. Hence $f_{2}^{*}\left(\ell_{1}-\ell_{2}\right)=f_{2}^{-1}\left[\ell_{1}\right]-f_{2}^{-1}\left[\ell_{2}\right]$ is numerically trivial since $\mathrm{SL}_{2^{-}}$operation on $\mathbf{P}^{1} \times \mathbf{P}^{1}=D$ is diagonal. Assume now that $(q \circ f)^{-1}[D]$ is exceptional for $f_{1}$. Then there exist a blow-down $\tilde{f}: \tilde{Y} \rightarrow Z$ collapsing $(q \circ f)^{-1}[D]$ to a curve $C \simeq \mathbf{P}^{1}$ on a non-singular projective variety. Putting $\tilde{D}=\left(q_{2} \circ f\right)^{-1}[D]$, the restriction $\tilde{f}: \mathbf{P}^{1} \times \mathbf{P}^{1} \simeq \tilde{D} \rightarrow C \simeq \mathbf{P}^{1}$ is a projection, say onto the first factor. Then $\tilde{f}_{*} f_{2}^{-1}\left[\ell_{1}\right]=a C, a>0$ and $\tilde{f}_{*} f_{2}^{-1}\left[\ell_{2}\right]=0$. Hence $C$ is numerically trivial. This is absurd since $Z$ is non-singular projective.

LEMMA (11.14). $\quad \mathcal{O}_{D_{\infty}}\left(D_{\infty}\right) \simeq \mathcal{O}_{\mathbf{P}_{1} \times \mathbf{P} 1}(2,-n)$.

Proof. Notice that in our notation, the restriction $p \mid D_{\infty} \rightarrow \mathbf{P}^{1}$ is the projection from $\mathbf{P}^{1} \times \mathbf{P}^{1}$ onto the second factor (cf. Table (11.9)). Let $\mathcal{O}_{D_{\infty}}\left(D_{\infty}\right) \simeq \mathcal{O}_{\mathbf{P}^{1} \times \mathbf{P}^{1}}(a, b)$. Then it follows from the definition of $X_{f}$ and that $D_{\infty}$ cuts on each fibre a divisor coming from the hyperplane section bundle of $\mathbf{P}^{3}$ hence $a=2$. Let us calculate $b$. For this purpose we recall the definition of $X_{f}$. We cover $\mathbf{P}^{1}$ by $2 \mathbf{A}^{1}$ 's $U_{0}, U_{1}$ in usual way: $\mathbf{P}^{1}=U_{0} \cup U_{1}$ and the points $t \in U_{0}$ and $1 / t \in U_{1}$ are identified. Gluing $V_{0}=U_{0} \times \mathbf{P}^{3}$ and $V_{1}=U_{1} \times \mathbf{P}^{3}$ together by identifying $\left(t,\left(x_{0}, x_{1}, x_{2}, x_{3}\right)\right) \in V_{0}$ and $\left(s,\left(y_{0}, y_{1}\right.\right.$, $\left.\left.y_{2}, y_{3}\right)\right) \in V_{1}$ if $t=1 / s, \quad x_{0}=y_{0}, \quad x_{1}=y_{1}, y_{2}=x_{2}, y_{3}=t^{n} x_{3}$, we thus get $\mathbf{P}(\mathcal{O} \oplus \mathcal{O} \oplus \mathcal{O} \oplus \mathcal{O}(n))$. Let $f(t)$ be a polynomial of degree $2 n$ with only simple roots. $X_{f}$ is defined locally on $V_{0}$ by $x_{0}^{2}+x_{1}^{2}+x_{2}^{3}+f(t) x_{3}^{2}=0$ and on $V_{1}$ by $y_{0}^{2}+y_{1}^{2}+y_{2}^{2}+f(t)(1 / 2 n) y_{3}^{2}=0$. Therefore $D_{\infty}$ is defined by $x_{3}=0$ on $V_{0} \cap X_{f}$ any $y_{3}=0$ on $V_{1} \cap X_{f}$. Now we put $\left.\ell=\{(t,(1,0,0,0))\} \in V_{0} \mid t \in k\right\}$ 
$\cup\left\{(s,(1,0,0,0)) \in V_{1} \mid s \in k\right\} . \quad \ell$ is a section of $p \mid D_{\infty}: D_{\infty} \rightarrow \mathbf{P}^{1}$ and $b$ is the degree of the line bundle $\mathcal{O}\left(D_{\infty}\right) \otimes \mathcal{O}_{\ell}$ on $\ell$. On $V_{0} \cap\left\{x_{0} \neq 0\right\} D_{\infty}$ is defined by $x_{3} / x_{0}$ and on $V_{1} \cap\left\{y_{0} \neq 0\right\}$ by $y_{3} / y_{0}=0$. Thus $\mathcal{O}\left(D_{\infty}\right) \otimes \mathcal{O}_{\ell}$ is defined by the transition function $\left(y_{3} / y_{0}\right) /\left(x_{3} / x_{0}\right)=y_{3} / x_{3}=t^{n}$ and hence $\mathcal{O}\left(D_{\infty}\right) \otimes \mathcal{O}_{\ell}$ is isomorphic to $\mathcal{O}_{\mathbf{P}}(-n)$. Therefore $b=-n$.

Lemma (11.15). The exceptional divisor for $\psi_{01}$ is exceptional for $q \circ f_{2}$.

Proof. Lemma (11.13) shows that the proper transform of a smooth fibre of $p$ is not exceptional for $f_{1}$. In view of Lemma (11.11) we have to show that the proper transform of one of the following divisors can not be exceptional for $f_{1}$ : (1) singular fibres for $p$, (2) $D_{\infty}$. In fact let $D$ be a singular fibre of $p$. $q^{*} D=q^{-1}[D]+2 E$ with $q^{-1}[D] \simeq \mathrm{F}_{2}, E \simeq \mathbf{P}^{2}$ by Lemma (11.8). Let $\ell$ be the fibre of the ruled surface $q^{-1}[D] \simeq F_{2}$. Since $D$ is a fibre, $0=\left(\ell \cdot q^{*}(\right.$ fibre of $\left.p)\right)=\left(\ell \cdot q^{*} D\right)=\left(\ell \cdot q^{-1}[D]+2 E\right)=\left(\ell \cdot q^{-1}[D]\right)$ $+2(\ell . E)=\left(\ell \cdot q^{-1}[D]\right)+2$ by Lemma (11.8). Therefore $\mathcal{O}\left(q^{-1}[D]\right) \otimes \mathcal{O}_{\ell} \simeq$ $\mathcal{O}_{\mathrm{P}_{1}}(-2)$ and hence the restriction of $\mathcal{O}\left(q \circ f_{2}^{-1}[D]\right)$ on a fibre of the ruled surface $\left(f_{2} \circ q\right)^{-1}[D] \simeq \mathrm{F}_{2}$ is $\mathcal{O}_{\mathrm{P}_{1}}(-k), k \geq 2$ and can not be exceptional for $\psi_{01}$. By Lemma (11.14) $\mathcal{O}_{D_{\infty}}\left(D_{\infty}\right)=(2,-n)$. Therefore putting $D^{\prime}=$ $\left(f_{2} \circ q\right)^{-1}\left[D_{\infty}\right], \mathcal{O}_{D^{\prime}}\left(D^{\prime}\right)=(2,-b)$ with $b \geq n$ since $\mathrm{SL}_{2}$ operates on $D_{\infty} \simeq$ $\mathbf{P}^{1} \times \mathbf{P}^{1}$ hence on $\left(\chi_{i m} \circ q\right)^{-1}\left[D_{\infty}\right]$ through the first factor. Thus $D^{\prime}$ can not be exceptional for $\psi_{01}$.

Proposition (11.16). $q \circ f: Y \cdots \rightarrow X_{f}$ is a blow-up morphism or $Y$ is an $\mathrm{SL}_{2}$-equivariant blow-up of $\left(\mathrm{SL}_{2}, X_{f}\right)$.

Proof. It follows from Lemma (11.15) the exceptional divisor for $\psi_{01}$ is an exceptional divisor for $f_{1} \circ q$ and no exceptional divisor is isomorphic to $\mathbf{P}^{1} \times \mathbf{P}^{1}$ by Lemma (11.11).

TheOREM (11.17). $\quad\left(\mathrm{SL}_{2}, X_{f}\right)$ is the unique minimal element of $\mathscr{C}(\mathrm{J} 12 ; \pi)$ and any other element of $\mathscr{C}(\mathrm{J} 12 ; \pi)$ is an equivariant blow-up of $\left(\mathrm{SL}_{2}, X_{f}\right)$.

The following example shows that the case (3.3.4), [Mo] occurs in $\mathscr{C}(\mathrm{J} 12 ; \pi)$.

EXAMPLe (11.18). Let $p^{-1}(t)=S \subset \mathbf{P}^{3}, t \in \mathbf{P}^{1}$ be a singular fibre of $\rho: X_{f} \rightarrow \mathbf{P}^{1}$. Let $m$ be a line in $\mathbf{P}^{3}$ lying on the singular quadric $S$. Regarding $\ell$ as a 1-cycle on $X_{f}$, we have $\left(m \cdot D_{\infty}\right)=1,\left(m \cdot p^{*} \mathcal{O}_{\mathbf{P} 1}(1)\right)=0$. Denoting by $\ell$ a fibre of $D_{\infty} \simeq \mathbf{P}^{1} \times \mathbf{P}^{1} \rightarrow \mathbf{P}^{1}$ with $\left(\ell \cdot p^{*} \mathcal{O}_{\mathbf{P}}(1)\right)=1$ as in Lemma (11.14). Let us denote by $Y \mathbf{P}(\mathscr{O} \oplus \mathcal{O} \oplus \mathcal{O} \oplus \mathcal{O}(n))$, by $\tilde{p}: Y \rightarrow \mathbf{P}^{1}$ the 
projection and by $\tilde{D}_{\infty}$ the divisor on $Y$ defined as follows: using the notation of the Proof of Lemma (11.14), $\tilde{D}_{\infty}$ is defined on $V_{0}$ by $x_{3}=0$ and on $V_{1}$ by $y_{3}=0$ so that $\tilde{D}_{\infty} \cap X_{f}=D_{\infty}$. We need one more divisor $\tilde{D}$ on $Y$ which is defined on $V_{0}$ by $x_{0}=0$ and on $V_{1}$ by $y_{0}=0$. By simple local calculations, we get the following.

$$
\begin{aligned}
& \tilde{p}_{*} \mathcal{O}_{Y}\left(\tilde{D}_{\infty}\right) \simeq \mathcal{O}_{\mathbf{P} 1}^{\oplus 3} \oplus \mathcal{O}_{\mathbf{P} 1}(-n) \\
& \tilde{p}_{*} \mathcal{O}_{Y}(\tilde{D}) \simeq \mathcal{O}_{\mathbf{P} 1}^{\oplus 3} \oplus \mathcal{O}_{\mathbf{P}}(n) .
\end{aligned}
$$

$\mathcal{O}_{Y}(\tilde{D})$ is generated by its global sections.

Let us content ourselves with checking (11.15.2) and (11.15.3) since (11.15.1) is proved by the same method. Using the notation of the Proof of Lemma (11.14), if we calculate $\tilde{p}_{*}\left(\mathcal{O}_{Y}(D)\right)$ locally for $U_{0} \times \mathbf{P}^{3} \rightarrow U_{0}$, we get $\tilde{p}_{*}\left(\mathcal{O}_{Y}(\tilde{D})\right) \mid U_{0}$ is a free $k[t]$-module

$$
\left\{a_{0}(t)+a_{1}(t) \frac{x_{1}}{x_{0}}+a_{2}(t) \frac{x_{2}}{x_{0}}+a_{3}(t) \frac{x_{3}}{x_{0}} \mid a_{i}(t) \in k[t], 1 \leq i \leq 3\right\} .
$$

Similarly on $U_{1}$,

$$
\begin{aligned}
& \tilde{p}_{*} \mathcal{O}_{Y}(\tilde{D}) \mid U_{1} \\
& \quad \simeq\left\{b_{0}(s)+b_{1}(s) \frac{y_{1}}{y_{0}}+b_{2}(s) \frac{y_{2}}{y_{0}}+b_{3}(s) \frac{y_{3}}{y_{0}} \mid b_{i}(s) \in k[s], 0 \leq i \leq 3\right\} .
\end{aligned}
$$

Since $t=1 / s, x_{0}=y_{0}, x_{1}=y_{1}, y_{2}=x_{2}, y_{3}=t^{n} x_{3}$, the identification of these 2 free modules on the intersection $U_{0} \cap U_{1}$ gives $\mathcal{O}^{\oplus 3} \oplus \mathcal{O}(n)$. Thus the set of global sections

$$
\begin{array}{r}
H^{0}\left(Y, \mathcal{O}_{Y}(\tilde{D})\right) \simeq\left\{a_{0}+a_{1} \frac{x_{1}}{x_{0}}+a_{2} \frac{x_{2}}{x_{0}}+a_{3}(t) \frac{x_{3}}{x_{0}} \mid a_{i} \in k \text { for } 0 \leq i \leq 2,\right. \\
\left.a_{3}(t) \in k[t] \text { with deg } a_{3}(t) \leq n\right\} \\
=\left\{b_{0}+b_{1} \frac{y_{1}}{y_{0}}+b_{2} \frac{y_{1}}{y_{0}}+b_{3}(s) \frac{y_{1}}{y_{0}} \mid b_{i} \in k \text { for } 0 \leq i \leq 2,\right. \\
\left.b_{3}(s) \in k[s] \text { with deg } b_{3}(s) \leq n\right\} .
\end{array}
$$

This shows (11.15.3).

It follows from (11.15.1), (11.15.2) and (11.15.3) that $\mathcal{O}_{\tilde{Y}}(\tilde{D}) \simeq \mathcal{O}_{\tilde{Y}}\left(\tilde{D}_{\infty}\right) \otimes$ $\tilde{p}^{*} \mathcal{O}_{\mathbf{P} 1}(n)$ and $\mathcal{O}_{\tilde{Y}}\left(\tilde{D}_{\infty}\right) \otimes \tilde{p}^{*} \mathcal{O}_{\mathbf{P} 1}(n)$ is generated by its global sections. In particular the restriction $\mathcal{O}_{\tilde{Y}}\left(\tilde{D}_{\infty}\right) \otimes \tilde{p}^{*} \mathcal{O}_{\mathbf{P} 1}(n) \otimes \mathcal{O}_{X_{f}} \simeq \mathcal{O}_{\tilde{X}_{f}}\left(D_{\infty}\right) \otimes \tilde{p}^{*} \mathcal{O}_{\mathbf{P} 1}(n)$ onto $X_{f}$ is generated by its global sections. Now we can show that $\mathbf{R}_{+} m+$ 
$\mathbf{R}_{+} \ell$ coincides with the cone $N E\left(X_{f}\right)$ spanned by the effective 1-cycles on $X_{f}$ (see the section 1). In fact by Lemma (11.14) $\mathbf{R} m+\mathbf{R} \ell=N\left(X_{f}\right)$ since we know $\left(m \cdot p^{*} \mathscr{O}_{\mathbf{P}_{1}}(1)\right)=0,\left(\ell \cdot p^{*} \mathcal{O}_{\mathbf{P}_{1}}(1)\right)=1$. It is sufficient to show that for $a, b \in \mathbf{R} a m+b \ell$ is numerically equivalent to an effective 1-cycle on $X_{f}$ if and only if $a, b \geq 0$. As the if part is evident, we have to show "only if" part. Assume that $a m+b \ell$ is numerically equivalent to an effective 1-cycle. Since $\mathcal{O}\left(D_{\infty}\right) \otimes p^{*} \mathcal{O}_{\mathbf{P}_{1}}(n)$ is generated by its global sections, we have $0 \leq\left(a m+b \ell . \mathcal{O}\left(D_{\infty}\right) \otimes p^{*} \mathcal{O}_{\mathbf{P} 1}(n)\right)=a\left(m . D_{\infty}\right)+b\left(\ell . D_{\infty}\right)+b\left(\ell \cdot p^{*} \mathcal{O}_{\mathrm{P}_{1}}(n)\right)$ $=a-n b+n b=a$ by Lemma (11.14). As $p^{*} \mathcal{O}_{\mathrm{P} 1}(n)$ is also generated by its global sections, $0 \leq\left(a m+b \ell \cdot p^{*} \mathscr{O}_{\mathrm{P}_{1}}(1)\right)=b$ as wanted.

Since by the adjunction formula $\mathcal{O}\left(K_{X_{f}}\right) \simeq \mathcal{O}_{Y}\left(K_{Y}+X_{f}\right) \otimes \mathcal{O}_{X_{f}} \simeq$ $\mathcal{O}_{X_{j}}\left(-2 D_{\infty}\right) \otimes p^{*} M$ for some line bundle $M$ over $\mathbf{P}^{1},\left(\ell . K_{X_{f}}\right)=-2$. Hence $\mathbf{R}_{+} \ell$ is an extremal ray. Let us now blow up $X_{f}$ at $C=S \cap D_{\infty}: \phi: Z \rightarrow$ $X_{f}$.
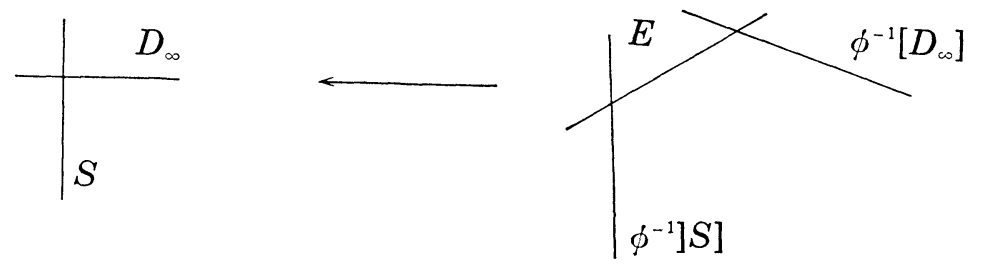

We denote by $E$ the exceptional divisor. Then $K_{Z}=\phi^{*} K_{X_{f}}+E$ and

$$
\begin{aligned}
\left(\phi^{-1}[\ell] \cdot K_{Z}\right) & =\left(\phi^{-1}[\ell] \cdot \phi^{*} K_{X_{f}}+E\right)=\left(\phi^{-1}[\ell] \cdot \phi^{*} K_{X_{f}}\right)+\left(\phi^{-1}[\ell] . E\right) \\
& =\left(\phi_{*} \phi^{-1}[\ell] \cdot K_{X_{f}}\right)+1, \text { by the projection formula, } \\
& =\left(\ell \cdot K_{X_{f}}\right)+1=-2+1=-1 .
\end{aligned}
$$

Hence $\mathbf{R}_{+} \dot{\phi}^{-1}[\ell]$ is an extremal ray. Moreover since

$$
\begin{aligned}
&\left(\phi^{-1}[\ell] \cdot \phi^{-1}[S]\right)+1=\left(\phi^{-1}[\ell], \phi^{-1}[S]+E\right)=\left(\phi^{-1}[\ell] \cdot \phi^{*} S\right) \\
&=\left(\phi_{*} \phi^{-1}[\ell] . S\right)=(\ell . S)=0, \quad\left(\phi^{-1}[\ell] \cdot \phi^{-1}[S]\right)=-1 .
\end{aligned}
$$

Since $S$ is the singular quadric in $\mathbf{P}^{3}$, this shows that on $\left(\mathrm{SL}_{2}, W\right) \in$ $\mathscr{C}(\mathrm{J} 12 ; \pi), \mathbf{R}_{+} \phi^{-1}[\ell]$ is an example of an extremal ray giving rise to case (3.3.4). 


\section{BIBLIOGRAPHY}

[A] Atiyah, M. F., Complex fibre bundles and ruled surfaces, Proc. London Math. Soc. (3), 5 (1955), 407-434.

[Be] Beauville, A., Variété de Prym et Jacobiennes intermédiaires, Ann. Sci. Ec. Norm. Sup., 4e série, t. 10 (1977), 304-392.

[Bo] Borel, A., Linear algebraic groups, Benjamin, New York, 1969.

[Da] Danilov, V. I., Decomposition of certain birational morphisms, Math. USSR Izv., 16 (1981), 419-429.

[De] Demazure, M., Sous-groupes algébriques de rang maximum du groupe de Cremona, Ann. Sci. Ec. Norm. Sup., 4e série, t. 3 (1970), 507-588.

[EF] Enriques, F. e Fano, G., Sui gruppi di transformazioni cremoniane dello spazio, Annali di Matematica pura ed applicata, s. $2^{a}$ to. 15 (1897), 59-98.

[F] Fano, G., I gruppi di Jonquières generalizzati, Atti della R. Acc. di Torino, 33 (1898), 221-271.

[Hi] Hironaka, H., Resolution of singularities of an algebraic variety over a field of characteristic zero, Ann. of Math., 79 (1964), 109-326.

[Hu] Humphreys, J. E., Introduction to Lie algebra and representation theory, GTM 9, Springer-Verlag, Berlin-Heidelberg-New York, 1972.

[I1] Iskovskih, V. A., Fano threefolds I, Math. USSR Izv., 11 (1977), 485-527.

[I2] - Fano threefolds II, Math. USSR Izv., 12 (1978), 469-506.

[Man] Manin, Yu. I., Cubic forms, North-Holland, Amsterdam, 1974.

[Mar1] Maruyama, M., On classification of ruled surfaces, Lect. in Math., Dept. Math. Kyoto Univ. 3, Kinokuniya, Tokyo 1970.

[Mar2] —- On a family of algebraic vector bundles, Number Theory, Algebraic Geometry and Commutative Algebra, in honor of Y. Akizuki, Kinokuniya, Tokyo, 1973, 95-146.

[Mi] Miyanishi, M., Algebraic methods in the theory of algebraic threefolds, Advanced Studies in Pure Math., 1, 1983, Alg. Var. and An. Var., 69-99.

[Mo] Mori, S., Threefolds, whose canonical bundles are not numerically effective, Ann. of Math., 116 (1982), 133-176.

[MU] Mukai, S. and Umemura, H., Minimal rational threefolds, Algebraic geometry, Lecture Notes in Math., 1016, Springer-Verlag, Berlin-Heidelberg-New York, 1983, 490-518.

[Re] Reid, M., Canonical 3-folds, Journée de géométrie algébrique d'Angers, ed. A. Beauville, Sijthoff and Noordhoff, Alphen 1980, 273-310.

[Ro] Rosenlicht, M., Some basic theorems on algebraic groups, Amer. J. Math., 78 (1956), 401-443.

[Sc] Schwarzenberger, R. L. E., Vector bundles on the projective plane, Proc. London Math. Soc., (3) 11 (1961), 623-640.

[Su] Sumihiro, H., Equivariant completion, J. Math. Kyoto Univ., 14-1 (1974), 1-28.

[U1] Umemura, H., Sur les sous-groupes algébriques primitifs de groupe de Cremona à trois variables, Nagoya Math. J., 79 (1980), 47-67.

[U2] - Maximal algebraic subgroups of the Cremona group of three variables, Nagoya Math. J., 87 (1982), 59-78.

[U3] - On the maximal connected algebraic subgroups of the Cremona group I, Nagoya Math. J., 88 (1982), 213-246.

[U4] - On the maximal connected algebraic subgroups of the Cremona group II, Algebraic groups and related topics, Advanced Studies in Pure Math., 6, Kinokuniya-North-Holland, 1985, 349-436.

[U5] - Algebro-geometric problems arising from Painlevé's works, Algebraic and 
Topological theories-to the memory of Dr. T. Miyata, Kinokuniya, Tokyo, 1985, 467-495.

[Z] Zariski, O., Introduction to the problem of minimal models in the theory of algebraic surfaces, Math. Soc. Japan, Tokyo, 1958.

Department of Mathematics

Mie University

Tsu, 514

Japan

Current address :

Department of Mathematics

Faculty of Science

Kumamoto University

Kumamoto, 860

Japan 